\title{
CONNECTING TRANSITIONS IN GALAXY PROPERTIES TO REFUELING
}

\author{
Sheila J. Kannappan ${ }^{1}$, David V. Stark ${ }^{1}$, Kathleen D. Eckert ${ }^{1}$, Amanda J. Moffett ${ }^{1}$, Lisa H. Wei ${ }^{2,3}$, D. J. Pisano ${ }^{4,12}$, \\ Andrew J. Baker ${ }^{5}$, Stuart N. Vogel ${ }^{6}$, Daniel G. Fabricant ${ }^{2}$, Seppo Laine ${ }^{7}$, Mark A. Norris ${ }^{1,8}$, Shardha Jogee $^{9}$, \\ Natasha LePore ${ }^{10}$, LOREn E. Hough ${ }^{11}$, AND JenNifer Weinberg-Wolf ${ }^{1}$ \\ ${ }^{1}$ Department of Physics and Astronomy, University of North Carolina, 290 Phillips Hall CB 3255, Chapel Hill, NC 27599, USA; sheila@ physics.unc.edu \\ ${ }^{2}$ Harvard-Smithsonian Center for Astrophysics, 60 Garden Street MS-20, Cambridge, MA 02138, USA \\ ${ }^{3}$ Atmospheric and Environmental Research, 131 Hartwell Avenue, Lexington, MA 02421, USA \\ ${ }^{4}$ Department of Physics, West Virginia University, P.O. Box 6315, Morgantown, WV 26506, USA \\ ${ }^{5}$ Department of Physics and Astronomy, Rutgers, the State University of New Jersey, 136 Frelinghuysen Road, Piscataway, NJ 08854-8019, USA \\ ${ }^{6}$ Department of Astronomy, University of Maryland, College Park, MD 20742-2421, USA \\ ${ }^{7}$ Spitzer Science Center, Caltech, MS 220-6, Pasadena, CA 91125, USA \\ ${ }^{8}$ Max Planck Institut fur Astronomie, Konigstuhl 17, D-69117, Heidelberg, Germany \\ ${ }^{9}$ Department of Astronomy, University of Texas at Austin, Austin, TX 78712, USA \\ ${ }^{10}$ Department of Radiology, University of Southern California and Children's Hospital Los Angeles, 4650 W Sunset Boulevard, \\ MS\#81, Los Angeles, CA 90027, USA \\ ${ }^{11}$ Department of Physics, University of Colorado at Boulder, Boulder, CO 80309-0390, USA \\ Received 2012 August 8; accepted 2013 July 17; published 2013 October 15
}

\begin{abstract}
We relate transitions in galaxy structure and gas content to refueling, here defined to include both the external gas accretion and the internal gas processing needed to renew reservoirs for star formation. We analyze two $z=0$ data sets: a high-quality 200 galaxy sample (the Nearby Field Galaxy Survey, data release herein) and a volumelimited $\sim 3000$ galaxy sample with reprocessed archival data. Both reach down to baryonic masses $\sim 10^{9} M_{\odot}$ and span void-to-cluster environments. Two mass-dependent transitions are evident: (1) below the "gas-richness threshold" scale ( $\left.V \sim 125 \mathrm{~km} \mathrm{~s}^{-1}\right)$, gas-dominated quasi-bulgeless Sd-Im galaxies become numerically dominant; while (2) above the "bimodality" scale $\left(V \sim 200 \mathrm{~km} \mathrm{~s}^{-1}\right)$, gas-starved E/S0s become the norm. Notwithstanding these transitions, galaxy mass (or $V$ as its proxy) is a poor predictor of gas-to-stellar mass ratio $M_{\text {gas }} / M_{*}$. Instead, $M_{\mathrm{gas}} / M_{*}$ correlates well with the ratio of a galaxy's stellar mass formed in the last Gyr to its preexisting stellar mass, such that the two ratios have numerically similar values. This striking correspondence between past-averaged star formation and current gas richness implies routine refueling of star-forming galaxies on Gyr timescales. We argue that this refueling underlies the tight $M_{\text {gas }} / M_{*}$ versus color correlations often used to measure "photometric gas fractions." Furthermore, the threshold and bimodality scale transitions reflect mass-dependent demographic shifts between three refueling regimes-accretion-dominated, processing-dominated, and quenched. In this picture, gas-dominated dwarfs are explained not by inefficient star formation but by overwhelming gas accretion, which fuels stellar mass doubling in $\lesssim 1$ Gyr. Moreover, moderately gas-rich bulged disks such as the Milky Way are transitional, becoming abundant only in the narrow range between the threshold and bimodality scales.
\end{abstract}

Key word: galaxies: evolution

Online-only material: color figures, machine-readable table

\section{INTRODUCTION}

Galaxies grow both by merging and by fresh gas accretion. Hierarchical models that follow the merger histories of galaxies and their host dark matter halos successfully explain the largescale structure of the universe, yet these models have difficulty reproducing the relative abundance of disk-dominated versus bulge-dominated galaxies across a broad range of environments (e.g., Navarro \& White 1994; Abadi et al. 2003; D’Onghia \& Burkert 2004; Stewart et al. 2008; Martig et al. 2009). Broadly speaking, this failure reflects the disk-destroying nature of stellar-mass-dominated mergers (and can therefore be mitigated by "quiet" merger histories such as may be found in low-density environments; Weinzirl et al. 2009; Fontanot et al. 2011). ${ }^{13}$ On the other hand, gas-rich mergers are much less destructive and

\footnotetext{
${ }^{12}$ Adjunct Assistant Astronomer, National Radio Astronomy Observatory, P.O. Box 2, Green Bank, WV 24944, USA.

13 The failure may also be compounded by the extreme loss of gas angular momentum in some simulations, although implementing star formation feedback and/or higher mass and force resolution can counteract this problem (e.g., Weil et al. 1998; Governato et al. 2007).
}

may even help to build disks. Such mergers are expected to predominate at low galaxy masses and/or early epochs (e.g., Robertson et al. 2006; Hopkins et al. 2009; Stewart et al. 2009). Fresh gas accretion may also rebuild disks in low-mass E/SO merger remnants, potentially restoring late-type morphologies (e.g., Cox et al. 2001; Morganti et al. 2006; Stark et al. 2013).

Observations point to significant cold gas accretion onto galaxies (Sancisi et al. 2008 and references therein). Moreover, the dynamics of halo gas suggest that this gas can in principle provide the angular momentum needed for the rapid growth of disks (Stewart et al. 2011). Cosmological hydro simulations show large-scale "cold" $\left(\sim 10^{5}-10^{6} \mathrm{~K}\right)$ gas flows that travel along the filaments and walls of the cosmic web (e.g., Kereš et al. 2005). Observational signs of such flows have indeed been found (Zitrin \& Brosch 2008; Stanonik et al. 2009; Narayanan et al. 2010; see also Giavalisco et al. 2011; Churchill et al. 2012). It is not yet clear to what extent these flows remain cold or shock-heat upon halo entry (compare Nelson et al. 2013 versus Birnboim \& Dekel 2003 and Kereš et al. 2005). These details may affect angular momentum delivery as well as the onset of rapid accretion, which typically occurs when the cooling radius 
exceeds the virial radius, below a characteristic mass scale that depends on the model ( $\mathrm{Lu}$ et al. 2011). Regardless of these specifics, it is a general feature of recent models that cosmic gas accretion accounts for a larger percentage of galaxy growth in low-mass halos than can be attributed to merging. Thus, the physics of accretion can dramatically change the balance of bulges and disks within the hierarchical merging paradigm.

Clearly mass-dependent gas physics affects patterns of growth by both mergers and accretion, and transitions in gas physics may lie at the heart of understanding the disky morphologies and overall growth histories of galaxies. Two galaxy mass scales have been previously noted as important transition points in morphology, gas richness (defined as gas-to-stellar mass ratio in this paper), and star formation history (SFH): the "bimodality scale" and the "gas-richness threshold scale."

The bimodality scale is typically identified with stellar mass $M_{*} \sim 10^{10.5} M_{\odot}$, which corresponds to rotation velocity $V \sim 200 \mathrm{~km} \mathrm{~s}^{-1}$ (see Section 3 herein). This scale marks the crossover point in relative abundance of young disk-dominated versus old spheroid-dominated stellar populations (Kauffmann et al. 2003a). As traced by late-type versus early-type morphology, this transition appears to shift downward in mass over cosmic time (Bundy et al. 2005). Equivalently, the bimodality scale marks a shift in the relative number density of galaxies on the red and blue sequences in $u-r$ color versus stellar mass $M_{*}$ parameter space (Baldry et al. 2004), which are associated with "red and dead" galaxies that have a strong $4000 \AA$ break and blue star-forming systems, respectively. Active galactic nucleus (AGN) activity in early-type galaxies peaks up just below the bimodality scale in a population that may be evolving toward the red sequence, suggesting black hole growth in tandem with spheroid formation (Schawinski et al. 2010). The slope of the gas-phase metallicity versus $M_{*}$ relation flattens above the bimodality scale, indicating changes in the interplay of gas cooling/infall, gas consumption, and gas loss in metal-enriched outflows (Tremonti et al. 2004). Hot gas halos become common above the bimodality scale (Mulchaey \& Jeltema 2010, translating their $K$-band magnitudes to equivalent stellar masses), potentially enhancing the efficacy of AGN feedback (Dekel \& Birnboim 2006).

A second, lower-mass transition scale was previously highlighted by Dekel \& Silk (1986), in a scenario explaining lowmetallicity diffuse dwarf galaxies as the result of global gas loss caused by supernova winds acting in the shallow potential wells of $V \lesssim 100 \mathrm{~km} \mathrm{~s}^{-1}$ dark matter halos. More recent work has shown that such "blowaway" (as distinct from local "blowout") should occur only in much smaller halos, near $V \sim 30 \mathrm{~km} \mathrm{~s}^{-1}$ (Mac Low \& Ferrara 1999). Blowaway near $V \sim 100 \mathrm{~km} \mathrm{~s}^{-1}$ would in any case be hard to reconcile with the fact that "high-mass dwarf" galaxies are typically gas-rich rather than gas-poor (e.g., Bettoni et al. 2003; Kannappan 2004, hereafter K04). In fact, gas-dominated galaxies become typical of the blue sequence below $M_{*} \sim 10^{9.5-10} M_{\odot}$ (corresponding to $V \sim 125 \mathrm{~km} \mathrm{~s}^{-1}$; Section 3 herein), as gas fractions rise on both sequences (K04 ${ }^{14}$; Kannappan \& Wei 2008; Kannappan et al. 2009, hereafter KGB). We therefore refer to the $V \sim 125 \mathrm{~km} \mathrm{~s}^{-1}$ scale as the "gas-richness threshold scale," following KGB. Calculations by Dalcanton (2007) suggest that an increase in gas-richness is essential to explain changes in

\footnotetext{
14 Identifying this shift in gas richness with the threshold scale requires shifting the stellar-mass zero point from Bell et al. (2003) as used by K04 to coincide with that of Kauffmann et al. (2003a).
}

metallicity at the threshold scale (specifically, the drop in effective yields below $V \sim 125 \mathrm{~km} \mathrm{~s}^{-1}$ reported by Garnett 2002), and that a second essential ingredient is low star formation efficiency (in the sense of star formation rate divided by gas mass; we will revisit this concept in relation to cosmic accretion in Section 4.4.2).

In a separate study of edge-on, "bulgeless" disk galaxies, Dalcanton et al. (2004) reported another presumably related change in interstellar medium (ISM) physics at the threshold scale: thin concentrated dust lanes emerge abruptly above $V \sim 120 \mathrm{~km} \mathrm{~s}^{-1}$. Moreover, despite the authors' best efforts to select for bulgeless morphology, in practice their high-quality follow-up imaging reveals a small "three-dimensional" bulge in every sample galaxy above $V \sim 120 \mathrm{~km} \mathrm{~s}^{-1}$, suggesting a link between changes in gas physics and galaxy structure. The onset of inevitable bulges above the threshold scale (also seen by Bell 2008) occurs simultaneously with a sharp decline in the population of "blue-sequence E/S0s," identified by KGB as gas-rich merger remnants rebuilding disks (although highermass blue E/S0s are more often quenching, especially above the bimodality scale; Schawinski et al. 2009; KGB). Fisher \& Drory (2011) report transitions in bulge demographics at both the threshold and bimodality scales, with bulgeless galaxies dominant below $M_{*} \sim 10^{9.5} M_{\odot}$, pseudobulges dominant between $10^{9.5}$ and $10^{10.5} M_{\odot}$, and classical bulges/elliptical galaxies dominant above $10^{10.5} M_{\odot}$. Interestingly, the E/S0 mass-radius relation bifurcates into two loci below the threshold scale (e.g., KGB; Misgeld \& Hilker 2011).

Given the variety of physical processes and galaxy properties changing at the threshold and bimodality scales, these closely spaced mass scales have been conflated by multiple authors, including K04 and Dekel \& Birnboim (2006), and the blurring of the two has been further exacerbated by systematic differences in stellar-mass zero points between investigators (see Kannappan \& Gawiser 2007; Kannappan \& Wei 2008). Yet appreciating the distinction between the threshold and bimodality scales is of key interest, since we will demonstrate herein that only in the narrow mass range between the threshold and bimodality scales do galaxies like our Milky Wayintermediate between gas-dominated bulgeless disks and gas-starved spheroids-become typical in the galaxy population. An exploration of the transitions occurring at the threshold and bimodality scales may therefore shed light on our Galaxy's past and future.

In what follows we employ multi-wavelength data from two complementary samples described in Section 2 to explore structural and gas-richness $\left(M_{\mathrm{gas}} / M_{*}\right)$ transitions across the threshold and bimodality scales in Section 3. We show that notwithstanding these notable transitions, there is far greater scatter in $M_{\mathrm{gas}} / M_{*}$ versus galaxy mass than has been previously appreciated. In contrast, we demonstrate in Section 4 that $M_{\text {gas }} / M_{*}$ correlates in a surprisingly one-to-one fashion with a quantity we refer to as the long-term fractional stellar mass growth rate $\left(\mathrm{FSMGR}_{\mathrm{LT}}\right)$, which considers star formation integrated over the last Gyr and is defined such that it can exceed one over the unit of time, unlike a specific star formation rate (SSFR). We argue that the $\mathrm{FSMGR}_{\mathrm{LT}}-M_{\mathrm{gas}} / M_{*}$ correlation, and not the Kennicutt-Schmidt Law, underlies the tight observed relation between $M_{\mathrm{gas}} / M_{*}$ and ultraviolet/blue minus near-infrared colors (hereafter, U-NIR colors) previously reported by K04. Moreover, we propose that coordinated changes in morphology and gas richness as a function of $\mathrm{FSMGR}_{\mathrm{LT}}$ can be usefully understood in terms of changes in cosmic accretion and internal 

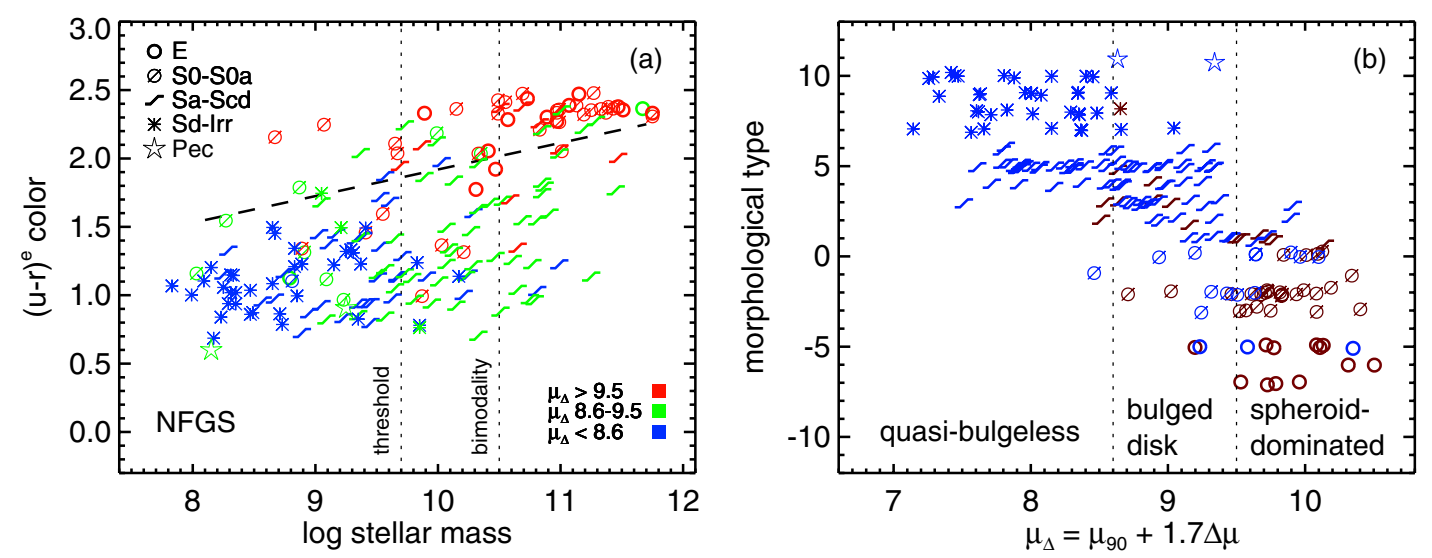

Figure 1. Morphological type, color, and stellar mass distribution of the Nearby Field Galaxy Survey (NFGS). (a) Distribution of NFGS galaxy morphologies in $(u-r)^{e}$ color vs. stellar mass $M_{*}$ parameter space, with symbol color corresponding to the $\mu_{\Delta}$ classes defined by the dividing lines in panel (b). Note that $(u-r)^{e}$ is a de-extincted color estimated by our stellar population modeling code and as such enhances the division between the red and blue sequences. However, it does not shift the basic locus of the red sequence significantly; rather, the bluer color of this locus compared to previous studies (e.g., Baldry et al. 2004) reflects improvements in our photometry compared to the SDSS pipeline, as described in Section 2.1.1 and Figure 2. (b) Calibration of the $\mu_{\Delta}$ parameter used to distinguish quasi-bulgeless, bulged-disk, and spheroid-dominated galaxies (see Section 2.1.1). Symbol color corresponds to the red-/blue-sequence division shown in panel (a). Small random offsets have been applied to the morphological types to separate points.

(A color version of this figure is available in the online journal.)

gas processing between three "refueling regimes": accretiondominated, processing-dominated, and quenched. Finally, we tie our results back to the threshold and bimodality scales, showing that these scales represent transitions in the relative numerical dominance of galaxies in the three regimes, likely tied to the halo mass dependence of cosmic accretion. Our results suggest a reevaluation of dwarf galaxies as not "inefficient" gas consumers but "overwhelmed" gas accretors, and of moderately gas-rich bulged disks like our Milky Way as not "normal" but "transitional" in the galaxy population.

\section{DATA AND METHODS}

Our analysis relies on two samples. The highest-quality $\mathrm{H}_{\mathrm{I}}$ data, multi-band photometry, and kinematic data come from the Nearby Field Galaxy Survey (NFGS; Jansen et al. 2000a, 2000b; Kannappan \& Fabricant 2001; Kannappan et al. 2002; Wei et al. 2010a), a broadly representative sample of $\sim 200$ galaxies spanning stellar masses $M_{*} \sim 10^{8}-10^{12} M_{\odot}$ and all morphologies. Better statistics are offered by a volume-limited sample of 〜3000 galaxies, hereafter the V3000 sample, with flux-limited $\mathrm{H}$ I data and partial kinematic information from the blind $21 \mathrm{~cm}$ Arecibo Legacy Fast ALFA (ALFALFA; Giovanelli et al. 2005; Haynes et al. 2011) survey, which we combine with reprocessed photometry from the Sloan Digital Sky Survey (SDSS; Aihara et al. 2011) Data Release 8 (DR8), the GALEX mission archive (Morrissey et al. 2007), and the Two Micron All Sky Survey (2MASS; Jarrett et al. 2000). We detail new and reprocessed archival data for the NFGS and V3000 samples below.

We assume $H_{0}=70 \mathrm{~km} \mathrm{~s}^{-1} \mathrm{Mpc}^{-1}$ and $d=\mathrm{cz} / H_{0}$ throughout this work. Neglecting $\Lambda$ introduces negligible errors at the low redshifts of our sample galaxies.

\subsection{The Nearby Field Galaxy Survey}

The NFGS was drawn from a $B$-selected parent survey, the CfA 1 Redshift Survey (Huchra et al. 1983), in approximate proportion to the luminosity function, and it preserves the CfA 1 survey's relative frequency of morphological types at each luminosity (Jansen et al. 2000b). The 196 galaxies in the NFGS obey an artificial luminosity-distance correlation imposed to ensure that their apparent diameters do not vary too much, as an observational convenience. Thus, large-scale environments are not uniformly sampled as a function of luminosity, although a wide variety of environments are represented, ranging from underdense regions to the Coma Cluster. Our analysis makes use of 190/196 galaxies in the NFGS, after rejection of 6 objects with nearly point-source morphology due to powerful AGNs or in one case a superposed star.

Figure 1 illustrates the color, stellar mass, and morphology distribution of these 190 galaxies. We note that the Hubble type classifications in Figure 1(a) are reliable but not perfect, due to the inclination-blind selection of the NFGS as well as the fact that when the galaxies were classified, Jansen et al. (2000b) allowed fairly large discrepancies between different classifiers to remain unresolved. Following Kannappan et al. (2009), we reclassify the polar ring galaxy UGC 9562 as an S0. We also reclassify the very round dust-lane galaxy NGC 3499 as an E. Figure 1(b) defines a new quantitative morphology metric used as a complementary diagnostic, discussed in Section 2.1.1 below.

\subsubsection{Photometry and Stellar Masses}

Our analysis combines $U B R$ photometry from Jansen et al. (2000b) and global (integrated slit-scanned) spectrophotometry from Jansen et al. (2000a) with our own custom reprocessed GALEX near-UV (NUV), SDSS ugriz, 2MASS JHK, and Spitzer IRAC $3.6 \mu \mathrm{m}$ photometry, provided in Table 1 . For the new photometric measurements we redetermine the position angle (P.A.) and axial ratio ( $b / a=$ ratio of semi-minor to semi-major axis) without reference to the Jansen et al. values, but our kinematic observations and analysis predate the new photometry and thus make use of measurements from Jansen et al. as detailed in Section 2.1.3 and Table 2. We adopt foreground Milky Way extinction corrections from Schlegel et al. (1998), except at $3.6 \mu \mathrm{m}$ where such corrections are negligible, and we adjust the Jansen et al. photometry and spectrophotometry to match.

Initially, each galaxy is run through an optical photometric pipeline that produces masks and freely determines elliptical apertures from a deep gri coadded image (K. D. Eckert et al., in preparation). The outer disk P.A. and axial ratio are then fixed 
Table 1

Photometry and Stellar Mass Estimates Table Description

\begin{tabular}{|c|c|}
\hline Column & Description \\
\hline 1 & NFGS ID number \\
\hline 2 & Object name \\
\hline 3 & $G A L E X$ NUV magnitude ${ }^{\mathrm{a}, \mathrm{b}}$ \\
\hline 4 & Error on GALEX NUV magnitude \\
\hline 5 & SDSS $u$ magnitude ${ }^{\mathrm{a}}$ \\
\hline 6 & Error on SDSS $u$ magnitude \\
\hline 7 & SDSS $g$ magnitude ${ }^{\mathrm{a}}$ \\
\hline 8 & Error on SDSS $g$ magnitude \\
\hline 9 & SDSS $r$ magnitude ${ }^{a}$ \\
\hline 10 & Error on SDSS $r$ magnitude \\
\hline 11 & SDSS $i$ magnitude ${ }^{\mathrm{a}}$ \\
\hline 12 & Error on SDSS $i$ magnitude \\
\hline 13 & SDSS $z$ magnitude ${ }^{a}$ \\
\hline 14 & Error on SDSS $z$ magnitude \\
\hline 15 & 2MASS $J$ magnitude ${ }^{a}$ \\
\hline 16 & Error on 2MASS $J$ magnitude \\
\hline 17 & 2MASS $H$ magnitude ${ }^{\mathrm{a}}$ \\
\hline 18 & Error on 2MASS $H$ magnitude \\
\hline 19 & 2MASS $K$ magnitude ${ }^{\mathrm{a}}$ \\
\hline 20 & Error on 2MASS $K$ magnitude \\
\hline 21 & Spitzer IRAC $3.6 \mu \mathrm{m}$ magnitude \\
\hline 22 & Error on Spitzer IRAC $3.6 \mu \mathrm{m}$ magnitude \\
\hline 23 & Log of stellar mass \\
\hline 24 & Log of stellar mass from $\mathrm{KGB}^{\mathrm{c}}$ \\
\hline 25 & SDSS $r$-band $50 \%$ light radius \\
\hline 26 & SDSS $r$-band $90 \%$ light radius \\
\hline
\end{tabular}

Notes.

a NUVugrizJHK magnitudes are reported with foreground extinction corrections determined from the Schlegel et al. (1998) dust maps using the extinction curves of O'Donnell (1994) and Cardelli et al. (1989) in the optical and UV, respectively.

b We assume an effective wavelength of $2271 \AA$ for the GALEX NUV filter.

c Stellar masses derived by Kannappan et al. (2009) (not used in this paper)

(This table is available in its entirety in a machinereadable form in the online journal. A portion is shown here for guidance regarding its form and content.)

and used to determine a second set of elliptical apertures imposed on all bands NUV+ugrizJHK+IRAC $3.6 \mu \mathrm{m}$, enabling robust extrapolation of total magnitudes, even at low signal-tonoise ratio $(\mathrm{S} / \mathrm{N})$. Final magnitudes and systematic errors are calculated from a comparison of different methods (exponential profile fitting, curve-of-growth, outer-disk color correction, and large-aperture magnitude) for each band. Our SDSS magnitudes are measured using the newly optimized background sky estimates provided with all DR8 images (though not actually incorporated in DR8 catalog photometry), as described in Blanton et al. (2011). The extended sky coverage of DR8 includes 177 of our 190 galaxies. For GALEX we adopt the background estimation provided by the mission pipeline (Morrissey et al. 2007), and the available coverage yields NUV magnitudes for 93 of the 190 galaxies.

In the near-IR, custom background subtraction is necessary. IRAC $3.6 \mu \mathrm{m}$ images are available for 107 of our sample galaxies, mainly courtesy of the Spitzer Survey of Stellar Structure in Galaxies (S4G; Sheth et al. 2010) and our own program targeting low-mass E/S0 galaxies (GO-30406, PI: Kannappan). We calculate $3.6 \mu \mathrm{m}$ magnitudes using the level 2 (PBCD) images produced by the Spitzer pipeline, which have a residual low-level, non-uniform background. To remove this, we mask the primary galaxy and any other bright objects in each frame, and the remaining image is convolved with a median filter of size roughly four times the optical size of the galaxy to create a smooth background map. This map is subtracted from the original image to yield the image on which we perform photometric measurements. The resulting magnitudes are in good agreement with those from Moffett et al. (2012), who applied a similar background subtraction technique, with typical differences of $\sim 0.04 \mathrm{mag}$. In turn, our 2MASS pipeline incorporates background subtraction methods optimized with reference to the IRAC imaging, which includes deep S4G imaging of dwarf galaxies. With this careful background subtraction and the imposition of ellipses determined from the optical profile fits (using the P.A.s and ellipticities from Jansen et al. 2000b for the 13 galaxies lacking SDSS data), we have found it possible to compute reliable $J H K$ magnitudes for the entire sample, albeit sometimes with large error bars. As in the optical, we use multiple extrapolation techniques to estimate systematic errors in the NIR, although we find that a curve-of-growth approach is generally most robust, especially for shallow 2MASS data (see Stark et al. 2013, hereafter S13, for further details on our NIR pipelines). It is noteworthy that our 2MASS magnitudes are well behaved in combined stellar population fits of optical and NIR data that include high-quality spectrophotometry and deep IRAC photometry.

Table 1 lists our NUV+ugrizJHK+3.6 $\mu \mathrm{m}$ magnitudes (including foreground Milky Way extinction corrections), with uncertainties determined by combining Poisson errors with systematic errors from profile extrapolation. The IRAC magnitude errors include an extra $10 \%$ uncertainty associated with the "aperture corrections" required for profiles extrapolated to infinity, which we have applied as prescribed by the Spitzer IRAC instrument handbook. ${ }^{15}$

As illustrated in Figure 2, our $u-r$ colors are systematically $\sim 0.2$ mag bluer than those determined from SDSS catalog photometry, which is also evident in the fact that the red sequence in Figure 1 is $\sim 0.2 \mathrm{mag}$ bluer than in previous studies using SDSS catalog data. However, the same figure shows that our colors are in close agreement with those determined by Jansen et al. (2000b). We attribute about half of the offset between our $u-r$ colors and those from the SDSS catalog to the improved sky subtraction of Blanton et al. (2011), who demonstrate that their new protocol will yield $\sim 0.1$ mag bluer colors for galaxies with $\log r_{50} \gtrsim 1.4$ (marked in Figure 2). However, we measure greater color differences than expected from the new sky subtraction alone. Some discrepancies certainly reflect catastrophic failures of the SDSS pipeline (a few so extreme they lie outside the plot boundaries), but some likely also reflect the fact that we (like Jansen et al. 2000b) measure magnitudes by methods that permit color gradients, whereas the SDSS model magnitude algorithm enforces a common profile in all bands, which is determined in the $r$ band. ${ }^{16}$ In the case of massive early-type galaxies, for which the centers are redder than the outskirts (e.g., La Barbera et al. 2010), the SDSS model magnitude methodology would be expected to produce an "overly red" red sequence.

Stellar masses and other stellar population parameters are estimated using a variant of the code described in Kannappan \& Gawiser (2007) and improved by KGB, which simultaneously

\footnotetext{
15 http://irsa.ipac.caltech.edu/data/SPITZER/docs/irac/

iracinstrumenthandbook/

16 http://www.sdss.org/dr7/algorithms/photometry.html\#mag_model
} 
Table 2

Kinematic and H I Data for the NFGS

\begin{tabular}{|c|c|c|c|c|c|c|c|c|c|c|c|c|}
\hline ID & $\begin{array}{l}\text { P.A. }^{a} \\
\left({ }^{\circ}\right)\end{array}$ & $\begin{array}{l}i^{\mathrm{b}} \\
\left(^{\circ}\right)\end{array}$ & $\begin{array}{c}V_{\mathrm{pmm}}{ }^{\mathrm{c}} \\
\left(\mathrm{km} \mathrm{s}^{-1}\right)\end{array}$ & $\begin{array}{l}r_{e}{ }^{\mathrm{d}} \\
\left({ }^{\prime \prime}\right)\end{array}$ & $\begin{array}{c}\text { Extent } \\
\left({ }^{\prime \prime}\right)\end{array}$ & $\begin{array}{l}\text { Asym. } \\
(\%)\end{array}$ & $\begin{array}{c}\mathrm{W}_{50} \mathrm{e}^{\mathrm{e}} \\
\left(\mathrm{km} \mathrm{s}^{-1}\right)\end{array}$ & $\begin{array}{c}\sigma_{r_{e / 4}} \\
\left(\mathrm{~km} \mathrm{~s}^{-1}\right)\end{array}$ & $\begin{array}{c}V_{*}{ }^{\mathrm{f}} \\
\left(\mathrm{km} \mathrm{s}^{-1}\right)\end{array}$ & $\begin{array}{c}V^{\mathrm{g}} \\
\left(\mathrm{km} \mathrm{s}^{-1}\right)\end{array}$ & $\begin{array}{l}\log M_{\mathrm{HI}_{\mathrm{I}}}{ }^{\mathrm{r}} \\
\left(\log M_{\odot}\right)\end{array}$ & $\begin{array}{c}\text { H I Range } \\
\left(\mathrm{km} \mathrm{s}^{-1}\right)\end{array}$ \\
\hline 1 & $160 / 160$ & 39.9 & $\cdots$ & 7.1 & $\ldots$ & $\ldots$ & $\ldots$ & $133 \pm 10$ & 120 & $188 \pm 14^{s d}$ & $<8.32$ & $4661-4904$ \\
\hline 2 & $10 / 10$ & 56.9 & 52 & 12.1 & 13.6 & 4.8 & [W10a] & $22 \pm 8^{1}$ & 66 & $99 \pm 19^{s r}$ & $\mathrm{~W} 10 \mathrm{a}^{\ddagger}$ & $\ldots$ \\
\hline 3 & $55 / 48$ & 20.2 & $\ldots$ & 28.8 & $\ldots$ & $\ldots$ & W10a & $188 \pm 11$ & $\ldots$ & $266 \pm 16^{s d}$ & W10a & $\ldots$ \\
\hline 4 & $168 / 160$ & 0.0 & 58 & 11.7 & 22.7 & 2.9 & W10a & $61 \pm 8^{1}$ & $\ldots$ & {$[87 \pm 12]^{s d}$} & W10a & $\ldots$ \\
\hline 5 & $24 / 25$ & 78.0 & 217 & 32.1 & 47.6 & 5.3 & W10a & $106 \pm 10^{1}$ & $\ldots$ & $221 \pm 11^{i r}$ & W10a & $\ldots$ \\
\hline 7 & $-/ 48$ & 34.3 & $\ldots$ & 32.3 & $\ldots$ & $\ldots$ & W10a & $316 \pm 18$ & $\ldots$ & $447 \pm 25^{s d}$ & W10a & $\ldots$ \\
\hline 8 & $24 / 25$ & 62.1 & 173 & 10.6 & 22.7 & 1.3 & W10a & $132 \pm 10^{1}$ & 171 & $215 \pm 28^{s r}$ & W10a & $\ldots$ \\
\hline 10 & $110 * / 110 *$ & 0.0 & $\ldots$ & 6.6 & $\ldots$ & $\ldots$ & $\ldots$ & $174 \pm 11$ & 17 & $247 \pm 16^{s d}$ & $<9.02^{\mathrm{k}}$ & 5094-5339 \\
\hline 11 & $-/ 90$ & 27.9 & $\ldots$ & 4.5 & $\ldots$ & $\ldots$ & {$[54 \pm 40]$} & $115 \pm 9$ & 28 & $163 \pm 13^{s d}$ & $8.62 \pm 0.20^{\dagger}$ & $5322-5471$ \\
\hline 12 & $90 / 90$ & 51.4 & 154 & 9.5 & 12.5 & 1.1 & {$[318 \pm 42]$} & $121 \pm 12^{1}$ & 163 & $232 \pm 31^{s r}$ & $9.67 \pm 0.10^{\dagger}$ & $9650-10716$ \\
\hline 13 & $122.5 /-$ & 0.0 & [37] & 23.2 & 23.8 & 9.0 & [W10a] & $\ldots$ & $\ldots$ & $\ldots$ & $\mathrm{W} 10 \mathrm{a}^{\ddagger}$ & $\ldots$ \\
\hline 14 & $-/ 44$ & 74.2 & $\ldots$ & 17.3 & $\ldots$ & $\ldots$ & $\ldots$ & $49 \pm 8^{1}$ & 76 & $97 \pm 17^{s r}$ & $<7.57$ & $2355-2548$ \\
\hline 15 & $2.5 / 5$ & 49.5 & 9 & 13.1 & 15.9 & 9.4 & W10a & $28 \pm 11^{1}$ & $\ldots$ & $68 \pm 10^{n r}$ & W10a & $\ldots$ \\
\hline 16 & $90 * / 90 *$ & 27.9 & 93 & 8.2 & 19.3 & 2.3 & W10a & $79 \pm 12^{1}$ & $\ldots$ & {$[289 \pm 146]^{n r}$} & W10a & $\ldots$ \\
\hline 17 & $168 /-$ & 39.9 & 87 & 6.0 & 20.4 & 5.8 & W10a & $\ldots$ & $\ldots$ & {$[150 \pm 44]^{i r}$} & W10a & $\ldots$ \\
\hline 18 & $30 /-$ & 37.8 & [11] & 61.7 & 29.5 & 37.0 & W10a & $\ldots$ & $\ldots$ & {$[44 \pm 10]^{i r}$} & W10a & $\ldots$ \\
\hline 19 & $40 / 44$ & 26.4 & 75 & 7.6 & 14.7 & 2.9 & W10a & $\ldots$ & $\ldots$ & {$[358 \pm 172]^{n r}$} & W10a & $\ldots$ \\
\hline 21 & $132.5 /-$ & 62.1 & 61 & 45.2 & 69.2 & 4.9 & W10a & $\ldots$ & $\ldots$ & $83 \pm 10^{i r}$ & W10a & $\ldots$ \\
\hline 22 & $-/ 135$ & 59.7 & $\ldots$ & 10.7 & $\ldots$ & $\ldots$ & {$[374 \pm 10]$} & $134 \pm 10$ & $\ldots$ & $190 \pm 14^{s d}$ & $9.69 \pm 0.02^{\dagger}$ & $3526-3829$ \\
\hline 23 & $54 / 55$ & 62.1 & 164 & 12.9 & 28.3 & 3.8 & [W10a] & $87 \pm 8^{1}$ & $\ldots$ & $189 \pm 22^{i r}$ & $\mathrm{~W} 10 \mathrm{a}^{\ddagger}$ & $\ldots$ \\
\hline 24 & $132.5 * /-$ & 17.3 & 39 & 22.2 & 36.3 & 12.1 & W10a & $\ldots$ & $\ldots$ & {$[187 \pm 61]^{n r}$} & W10a & $\ldots$ \\
\hline 25 & $43 / 43$ & 62.1 & 128 & 16.6 & 27.2 & 0.9 & W10a & $60 \pm 8^{1}$ & $\ldots$ & $163 \pm 19^{n r}$ & W10a & $\ldots$ \\
\hline 26 & $54 /-$ & 66.4 & 223 & 15.5 & 62.4 & 2.7 & W10a & $\ldots$ & $\ldots$ & $249 \pm 15^{n r}$ & W10a & $\ldots$ \\
\hline 27 & $70 * /-$ & 0.0 & 49 & 16.3 & 31.8 & 8.3 & W10a & $\cdots$ & $\cdots$ & $\ldots$ & W10a & $\ldots$ \\
\hline 28 & $12 /-$ & 71.1 & 50 & 29.4 & 30.6 & 4.9 & W10a & $\ldots$ & $\ldots$ & $67 \pm 10^{i r}$ & W10a & $\ldots$ \\
\hline 29 & $120 /-$ & 49.5 & [197] & 22.6 & 17.0 & 1.9 & W10a & $\ldots$ & $\ldots$ & $377 \pm 31^{n r}$ & W10a & $\ldots$ \\
\hline 30 & $70 / 70$ & 75.8 & $\ldots$ & 13.7 & $\ldots$ & $\ldots$ & {$[261 \pm 8]$} & $186 \pm 12$ & 205 & $262 \pm 17^{s d}$ & $9.88 \pm 0.01^{\dagger}$ & $3428-3850$ \\
\hline 32 & $45^{*} /-$ & 39.9 & 41 & 46.1 & 61.2 & 3.5 & W10a & $\ldots$ & $\ldots$ & {$[84 \pm 11]^{i r}$} & W10a & $\ldots$ \\
\hline 33 & $-/ 70$ & 24.1 & $\ldots$ & 11.4 & $\ldots$ & $\ldots$ & {$[84 \pm 3]$} & $177 \pm 14$ & $\ldots$ & $250 \pm 19^{s d}$ & $9.15 \pm 0.10$ & $6367-6529$ \\
\hline 34 & $90 * / 90 *$ & 0.0 & 113 & 8.9 & 19.3 & 2.3 & {$[203 \pm 6]$} & $122 \pm 11^{1}$ & 79 & {$[172 \pm 15]^{s d}$} & $10.24 \pm 0.03^{\dagger}$ & $8100-8418$ \\
\hline 35 & $110 / 110$ & 42.5 & $\ldots$ & 22.6 & $\ldots$ & $\ldots$ & {$[277 \pm 15]$} & $241 \pm 15$ & $\ldots$ & $341 \pm 21^{s d}$ & $9.10 \pm 0.06$ & $4598-4957$ \\
\hline 36 & $165 / 170$ & 78.0 & $\ldots$ & 19.8 & $\ldots$ & $\ldots$ & W10a & $170 \pm 11^{1}$ & $\ldots$ & $240 \pm 15^{s d}$ & W10a & $\ldots$ \\
\hline 37 & $1 / 170$ & 48.1 & 259 & 21.5 & 35.2 & 1.9 & W10a & $234 \pm 14^{1}$ & $\ldots$ & $348 \pm 35^{n r}$ & W10a & $\ldots$ \\
\hline 38 & $1 /-$ & 85.0 & 54 & 33.7 & 52.2 & 3.0 & [W10a] & $\ldots$ & $\ldots$ & $67 \pm 10^{i r}$ & $\mathrm{~W} 10 \mathrm{a}^{\ddagger}$ & $\ldots$ \\
\hline 39 & $150 / 151$ & 46.8 & 123 & 15.2 & 22.7 & 4.0 & W10a & $47 \pm 19^{1}$ & 99 & $194 \pm 39^{n r}$ & W10a & $\ldots$ \\
\hline 40 & $80 / 90$ & 54.7 & $\ldots$ & 40.9 & $\ldots$ & $\ldots$ & $\ldots$ & $\ldots$ & $\ldots$ & $\ldots$ & $<6.58$ & $522-675$ \\
\hline 41 & $130 /-$ & 82.4 & 53 & 25.2 & 31.8 & 6.9 & [W10a] & $\ldots$ & $\ldots$ & $66 \pm 10^{i r}$ & $\mathrm{~W} 10 \mathrm{a}^{\ddagger}$ & $\ldots$ \\
\hline 42 & $-/ 170$ & 54.7 & $\ldots$ & 45.6 & $\ldots$ & $\cdots$ & {$[146 \pm 86]$} & $122 \pm 9$ & $\ldots$ & $172 \pm 13^{s d}$ & $8.44 \pm 0.19$ & $2570-2930$ \\
\hline 43 & $13 / 14$ & 64.0 & 148 & 18.4 & 43.1 & 2.6 & W10a & $110 \pm 9^{1}$ & $\ldots$ & $174 \pm 11^{n r}$ & W10a & $\ldots$ \\
\hline 44 & $52 / 50$ & 27.9 & 59 & 6.7 & 18.1 & 5.4 & $159 \pm 4$ & $54 \pm 7^{1}$ & 24 & {$[170 \pm 86]^{n r}$} & $8.53 \pm 0.03$ & $1420-1665$ \\
\hline 45 & $115^{*} /-$ & 29.6 & 3 & 12.4 & 18.1 & 14.4 & W10a & $\ldots$ & $\ldots$ & {$[415 \pm 221]^{n r}$} & W10a & $\ldots$ \\
\hline 46 & $1 * /-$ & 31.7 & 77 & 10.9 & 19.3 & 5.6 & [W10a] & $\ldots$ & $\ldots$ & {$[167 \pm 94]^{i r}$} & $\mathrm{~W} 10 \mathrm{a}^{\ddagger}$ & $\ldots$ \\
\hline 47 & 76/- & 57.3 & {$[68]$} & 37.2 & 28.3 & 6.1 & W10a & $\ldots$ & $\ldots$ & $102 \pm 7^{n r}$ & W10a & $\ldots$ \\
\hline 48 & 130/- & 49.5 & 136 & 21.4 & 37.4 & 3.8 & W10a & $\ldots$ & $\ldots$ & $186 \pm 28^{i r}$ & W10a & $\ldots$ \\
\hline 49 & $103 / 100$ & 66.1 & 127 & 13.8 & 29.5 & 0.6 & W10a & $66 \pm 15^{1}$ & $\ldots$ & $146 \pm 12^{i r}$ & W10a & $\ldots$ \\
\hline 50 & $40 /-$ & 51.7 & 59 & 46.8 & 60.1 & 10.2 & W10a & $\ldots$ & $\ldots$ & $91 \pm 11^{i r}$ & W10a & $\ldots$ \\
\hline 51 & $103 /-$ & 62.1 & 61 & 25.1 & 31.8 & 1.6 & W10a & $\ldots$ & $\ldots$ & $82 \pm 11^{i r}$ & W10a & $\ldots$ \\
\hline 52 & $1 /-$ & 62.1 & 53 & 20.8 & 28.3 & 6.1 & [W10a] & $\ldots$ & $\cdots$ & $73 \pm 11^{i r}$ & $\mathrm{~W} 10 \mathrm{a}^{\ddagger}$ & $\cdots$ \\
\hline 53 & $144 /-$ & 54.7 & 78 & 36.8 & 64.6 & 5.8 & W10a & $\ldots$ & $\ldots$ & $123 \pm 6^{n r}$ & W10a & $\ldots$ \\
\hline 54 & $-/ 50$ & 81.2 & $\ldots$ & 13.6 & $\ldots$ & $\cdots$ & $\ldots$ & $195 \pm 11$ & 218 & $276 \pm 16^{s d}$ & $<8.97$ & $6913-7433$ \\
\hline 55 & $101 /-$ & 37.8 & 27 & 15.2 & 22.7 & 6.1 & [W10a] & $\ldots$ & $\ldots$ & {$[68 \pm 21]^{i r}$} & $\mathrm{~W} 10 \mathrm{a}^{\ddagger}$ & $\ldots$ \\
\hline 56 & $130 / 140$ & 44.5 & 64 & 18.7 & 29.5 & 3.1 & W10a & $36 \pm 10^{1}$ & $\ldots$ & $109 \pm 21^{n r}$ & W10a & $\ldots$ \\
\hline 57 & $1 /-$ & 67.2 & 55 & 41.7 & 76.0 & 9.4 & W10a & $\ldots$ & $\ldots$ & $74 \pm 3^{n r}$ & W10a & $\ldots$ \\
\hline 58 & $154 /-$ & 90.0 & 170 & 43.3 & 45.4 & 1.5 & W10a & $\ldots$ & $\ldots$ & $173 \pm 10^{i r}$ & W10a & $\ldots$ \\
\hline 59 & $110 / 110$ & 27.9 & 41 & 6.5 & 7.9 & 2.7 & $104 \pm 27$ & $38 \pm 10^{1}$ & 73 & {$[193 \pm 99]^{s r}$} & $8.60 \pm 0.05$ & $3100-3600$ \\
\hline 60 & $1 /-$ & 85.5 & 145 & 24.4 & 31.8 & 1.6 & W10a & $\ldots$ & $\ldots$ & $151 \pm 7^{n r}$ & W10a & $\ldots$ \\
\hline 61 & $114 /-$ & 59.7 & 169 & 15.3 & 30.6 & 2.4 & W10a & $\ldots$ & $\ldots$ & $200 \pm 18^{i r}$ & W10a & $\ldots$ \\
\hline 62 & $54 /-$ & 75.8 & 68 & 19.4 & 31.8 & 6.5 & W10a & $\ldots$ & $\ldots$ & $82 \pm 10^{i r}$ & W10a & $\ldots$ \\
\hline 63 & $40 /-$ & 84.6 & 71 & 18.1 & 31.8 & 5.5 & W10a & $\ldots$ & $\ldots$ & $82 \pm 10^{i r}$ & W10a & $\ldots$ \\
\hline 64 & $40 /-$ & 31.7 & 40 & 7.4 & 10.2 & 6.4 & W10a & $\ldots$ & $\ldots$ & {$[210 \pm 118]^{n r}$} & W10a & $\ldots$ \\
\hline 65 & $121 / 120$ & 84.8 & 333 & 23.6 & 36.3 & 1.4 & $708 \pm 7$ & $231 \pm 14^{1}$ & 306 & $355 \pm 4^{n r}$ & $10.16 \pm 0.03$ & $10367-11208$ \\
\hline 66 & $130 / 140$ & 0.0 & 95 & 9.1 & 23.8 & 2.6 & W10a & $78 \pm 8^{1}$ & $\ldots$ & {$[110 \pm 12]^{s d}$} & W10a & $\ldots$ \\
\hline 67 & $144 / 140$ & 26.4 & 136 & 15.4 & 26.1 & 7.1 & $279 \pm 3$ & $124 \pm 10^{1}$ & $\ldots$ & {$[319 \pm 154]^{i r}$} & $9.90 \pm 0.04$ & $7920-8242$ \\
\hline
\end{tabular}


Table 2

(Continued)

\begin{tabular}{|c|c|c|c|c|c|c|c|c|c|c|c|c|}
\hline ID & $\begin{array}{l}\text { P.A. }^{\mathrm{a}} \\
\left({ }^{\circ}\right)\end{array}$ & $\begin{array}{l}i^{\mathrm{b}} \\
\left(^{\circ}\right)\end{array}$ & $\begin{array}{c}V_{\mathrm{pmm}^{\mathrm{c}}} \\
\left(\mathrm{km} \mathrm{s}^{-1}\right)\end{array}$ & $\begin{array}{l}r_{e}^{\mathrm{d}} \\
\left({ }^{\prime \prime}\right)\end{array}$ & $\begin{array}{c}\text { Extent } \\
\left({ }^{\prime \prime}\right)\end{array}$ & $\begin{array}{c}\text { Asym. } \\
(\%)\end{array}$ & $\begin{array}{c}\mathrm{W}_{50}{ }^{\mathrm{e}} \\
\left(\mathrm{km} \mathrm{s}^{-1}\right)\end{array}$ & $\begin{array}{c}\sigma_{r_{e / 4}} \\
\left(\mathrm{~km} \mathrm{~s}^{-1}\right)\end{array}$ & $\begin{array}{c}V_{*}^{\mathrm{f}} \\
\left(\mathrm{km} \mathrm{s}^{-1}\right)\end{array}$ & $\begin{array}{c}V^{\mathrm{g}} \\
\left(\mathrm{km} \mathrm{s}^{-1}\right)\end{array}$ & $\begin{array}{l}\log M_{\mathrm{HI}_{\mathrm{I}}}^{\mathrm{h}} \\
\left(\log M_{\odot}\right)\end{array}$ & $\begin{array}{l}\text { Hi Range }{ }^{\mathrm{j}} \\
\left(\mathrm{km} \mathrm{s}^{-1}\right)\end{array}$ \\
\hline 68 & $172 / 170$ & 66.1 & 67 & 14.5 & 19.3 & 8.1 & $140 \pm 7$ & $\ldots$ & $\ldots$ & $85 \pm 13^{i r}$ & $7.89 \pm 0.02$ & $535-869$ \\
\hline 69 & $100 * / 100 *$ & 0.0 & 8 & 4.0 & 5.7 & 19.3 & $136 \pm 10$ & $46 \pm 11^{1}$ & 21 & {$[64 \pm 16]^{s d}$} & $9.64 \pm 0.02$ & $5644-6023$ \\
\hline 70 & 116/- & 86.6 & 102 & 31.3 & 45.4 & 2.0 & W10a & $\ldots$ & $\ldots$ & $111 \pm 10^{i r}$ & W10a & $\ldots$ \\
\hline 71 & $54 /-$ & 79.6 & 111 & 23.0 & 39.7 & 4.5 & W10a & $\ldots$ & $\ldots$ & $123 \pm 4^{n r}$ & W10a & $\ldots$ \\
\hline 72 & $-/ 50 *$ & $90(29.6)$ & $\ldots$ & 7.6 & $\ldots$ & $\ldots$ & {$[257 \pm 11]$} & $61 \pm 7^{1}$ & 49 & {$[133 \pm 74]^{s r}$} & $8.07 \pm 0.06$ & $1358-1725$ \\
\hline 73 & $165 /-$ & 85.5 & 86 & 42.9 & 88.5 & 6.4 & W10a & $\ldots$ & $\ldots$ & $96 \pm 10^{i r}$ & W10a & $\ldots$ \\
\hline 75 & $-/ 122$ & 56.0 & $\ldots$ & 14.8 & $\ldots$ & $\ldots$ & {$[245 \pm 60]$} & $99 \pm 8$ & $\ldots$ & $141 \pm 12^{s d}$ & $8.62 \pm 0.05$ & 934-1489 \\
\hline 76 & $90 /-$ & 63.6 & 107 & 43.8 & 62.4 & 4.6 & W10a & $\ldots$ & $\ldots$ & $128 \pm 11^{i r}$ & W10a & $\ldots$ \\
\hline 77 & $165 / 170$ & 69.3 & 163 & 27.0 & 65.8 & 1.0 & [W10a] & $74 \pm 12^{1}$ & $\ldots$ & $178 \pm 12^{i r}$ & W10 $\mathrm{a}^{\ddagger}$ & $\ldots$ \\
\hline 78 & $-/ 100$ & 45.6 & $\ldots$ & 8.1 & $\ldots$ & $\ldots$ & $\ldots$ & $195 \pm 13$ & $\ldots$ & $276 \pm 18^{s d}$ & $<8.87$ & $7368-7750$ \\
\hline 79 & $20 /-$ & $43.0(58.1)$ & 113 & 8.4 & 15.9 & 5.2 & {$[263 \pm 16]$} & $\ldots$ & $\ldots$ & $177 \pm 31^{i r}$ & $10.67 \pm 0.01^{\dagger}$ & $12500-13076$ \\
\hline 80 & $-/ 18$ & 62.1 & $\ldots$ & 20.6 & $\ldots$ & $\ldots$ & $\ldots$ & $88 \pm 7$ & $\ldots$ & $124 \pm 10^{s d}$ & $<6.62$ & 549-772 \\
\hline 81 & $165 / 170$ & 76.2 & 90 & 24.3 & 29.5 & 2.5 & W10a & $69 \pm 21^{1}$ & $\ldots$ & $103 \pm 11^{i r}$ & W10a & $\ldots$ \\
\hline 82 & 76/- & 74.2 & {$[151]$} & 23.1 & 20.4 & 3.9 & [W10a] & $\ldots$ & $\ldots$ & {$[161 \pm 12]^{i r}$} & $\mathrm{~W} 10 \mathrm{a}^{\ddagger}$ & $\ldots$ \\
\hline 83 & $125 / 125$ & 42.5 & 11 & 6.0 & 7.9 & 21.6 & [W10a] & $\ldots$ & 23 & $59 \pm 25^{s r}$ & $\mathrm{~W} 10 \mathrm{a}^{\ddagger}$ & $\ldots$ \\
\hline 84 & $11 /-$ & 52.1 & 51 & 64.6 & 72.6 & 4.1 & W10a & $\ldots$ & $\ldots$ & $83 \pm 4^{n r}$ & W10a & $\ldots$ \\
\hline 85 & $133 / 140$ & 75.4 & 131 & 19.3 & 26.1 & 3.2 & W10a & $56 \pm 11^{l}$ & $\ldots$ & $142 \pm 11^{i r}$ & W10a & $\ldots$ \\
\hline 86 & $-/ 170$ & 51.4 & $\ldots$ & 19.9 & $\ldots$ & $\ldots$ & $\ldots$ & $257 \pm 15$ & $\ldots$ & $363 \pm 21^{s d}$ & $<9.07$ & $9245-9786$ \\
\hline 87 & $122 / 122$ & 62.1 & [23] & 9.5 & 7.9 & 2.4 & $100 \pm 6$ & $49 \pm 9^{1}$ & $\ldots$ & {$[70 \pm 13]^{s d}$} & $8.65 \pm 0.02$ & $1489-1794$ \\
\hline 88 & $172 /-$ & 90.0 & 94 & 32.0 & 56.7 & 2.8 & W10a & $\ldots$ & $\ldots$ & $105 \pm 4^{n r}$ & W10a & $\ldots$ \\
\hline 89 & $80 /-$ & 37.8 & 46 & 37.9 & 54.4 & 11.2 & W10a & $\ldots$ & $\ldots$ & {$[95 \pm 13]^{i r}$} & W10a & $\ldots$ \\
\hline 90 & $54 /-$ & 29.6 & 115 & 9.2 & 22.7 & 4.0 & $171 \pm 5$ & $\ldots$ & $\ldots$ & {$[248 \pm 132]^{i r}$} & $10.64 \pm 0.01$ & $10660-11110$ \\
\hline 91 & $52 / 50$ & 80.2 & 106 & 25.6 & 53.3 & 1.9 & W10a & $\ldots$ & $\ldots$ & $116 \pm 10^{i r}$ & W10a & $\ldots$ \\
\hline 92 & $73 / 73$ & 66.1 & 48 & 7.6 & 11.3 & 0.9 & [W10a] & $40 \pm 14^{1}$ & 34 & $67 \pm 12^{i r}$ & W10a & $\ldots$ \\
\hline 93 & $18 / 18$ & 54.7 & [4] & 7.6 & 9.1 & 19.5 & $51 \pm 3$ & $\ldots$ & $\ldots$ & {$[31 \pm 8]^{n r}$} & $7.44 \pm 0.02$ & $682-824$ \\
\hline 94 & $40 /-$ & 31.7 & 60 & 23.1 & 44.2 & 4.8 & W10a & $\ldots$ & $\ldots$ & {$[143 \pm 24]^{n r}$} & W10a & $\ldots$ \\
\hline 95 & $131 /-$ & 65.4 & 82 & 36.3 & 45.4 & 7.7 & [W10a] & $\ldots$ & $\ldots$ & $102 \pm 11^{i r}$ & $\mathrm{~W} 10 \mathrm{a}^{\ddagger}$ & $\ldots$ \\
\hline 96 & $100 / 100$ & 45.6 & [24] & 5.6 & 5.7 & 20.7 & $102 \pm 10$ & $34 \pm 12^{1}$ & 14 & {$[71 \pm 21]^{n r}$} & $7.83 \pm 0.05$ & $992-1252$ \\
\hline 97 & $165 /-$ & 21.5 & [23] & 40.7 & 39.7 & 7.7 & W10a & $\ldots$ & $\ldots$ & {$[102 \pm 22]^{i r}$} & W10a & $\ldots$ \\
\hline 98 & $170 / 170$ & 79.4 & [93] & 15.5 & 11.3 & 3.0 & W10a & $\ldots$ & $\ldots$ & $136 \pm 6^{n r}$ & W10a & $\ldots$ \\
\hline 99 & 146/- & 29.0 & 82 & 39.5 & 61.2 & 3.1 & W10a & $\ldots$ & $\ldots$ & {$[189 \pm 31]^{i r}$} & W10a & $\ldots$ \\
\hline 100 & $90.5 / 100$ & 20.2 & 96 & 16.4 & 36.3 & 3.1 & W10a & $174 \pm 15^{1}$ & $\ldots$ & {$[304 \pm 114]^{i r}$} & W10a & $\ldots$ \\
\hline 101 & $40 * /-$ & 17.5 & 53 & 48.5 & 79.4 & 9.8 & W10a & $\ldots$ & $\ldots$ & {$[217 \pm 36]^{i r}$} & W10a & $\ldots$ \\
\hline 102 & $80 /-$ & 51.1 & 67 & 21.3 & 36.3 & 2.6 & [W10a] & $\ldots$ & $\ldots$ & $100 \pm 14^{i r}$ & $\mathrm{~W} 10 \mathrm{a}^{\ddagger}$ & $\ldots$ \\
\hline 103 & $1 / 6$ & 44.9 & 91 & 25.2 & 37.4 & 3.5 & [W10a] & $\ldots$ & $\ldots$ & $142 \pm 19^{i r}$ & $\mathrm{~W} 10 \mathrm{a}^{\ddagger}$ & $\ldots$ \\
\hline 104 & $172 /-$ & 77.3 & 81 & 25.2 & 44.2 & 3.9 & W10a & $\ldots$ & $\ldots$ & $94 \pm 10^{i r}$ & W10a & $\ldots$ \\
\hline 105 & $100 / 100$ & 62.1 & 11 & 8.4 & 10.2 & 6.7 & $73 \pm 4$ & $38 \pm 14^{1}$ & 31 & $54 \pm 18^{s r}$ & $8.81 \pm 0.01$ & $1389-1663$ \\
\hline 106 & $18 / 18$ & 72.2 & {$[46]$} & 16.8 & 7.9 & 3.2 & {$[253 \pm 39]$} & $66 \pm 8^{1}$ & $\ldots$ & {$[94 \pm 11]^{s d}$} & $8.51 \pm 0.04^{\dagger}$ & $873-1150$ \\
\hline 107 & $165 /-$ & 84.3 & 100 & 30.4 & 45.4 & 3.4 & W10a & $\ldots$ & $\ldots$ & $109 \pm 10^{i r}$ & W10a & $\ldots$ \\
\hline 108 & $80 /-$ & 53.6 & [49] & 16.9 & 13.6 & 3.6 & [W10a] & $\ldots$ & $\ldots$ & {$[77 \pm 13]^{i r}$} & $\mathrm{~W} 10 \mathrm{a}^{\ddagger}$ & $\ldots$ \\
\hline 109 & $80 /-$ & 51.4 & 72 & 18.2 & 35.2 & 3.2 & W10a & $\ldots$ & $\ldots$ & $106 \pm 16^{i r}$ & W10a & $\ldots$ \\
\hline 110 & $40 /-$ & 69.3 & 75 & 18.4 & 34.0 & 7.6 & W10a & $\ldots$ & $\ldots$ & $93 \pm 8^{n r}$ & W10a & $\ldots$ \\
\hline 111 & $146 /-$ & 0.0 & 19 & 61.5 & 70.3 & 9.3 & W10a & $\ldots$ & $\ldots$ & $\ldots$ & W10a & $\ldots$ \\
\hline 112 & $40 /-$ & 81.2 & 118 & 26.9 & 44.2 & 2.8 & W10a & $\ldots$ & $\ldots$ & $126 \pm 10^{i r}$ & W10a & $\ldots$ \\
\hline 113 & $108 / 110$ & 68.8 & {$[25]$} & 40.7 & 37.4 & 6.1 & W10a & $\ldots$ & $\ldots$ & {$[43 \pm 10]^{i r}$} & W10a & $\ldots$ \\
\hline 114 & $40 /-$ & 77.3 & 146 & 26.3 & 46.5 & 1.9 & W10a & $\ldots$ & $\ldots$ & $154 \pm 11^{i r}$ & W10a & $\ldots$ \\
\hline 115 & $-/ 110 *$ & 26.4 & $\ldots$ & 15.6 & $\ldots$ & $\ldots$ & {$[227 \pm 14]$} & $248 \pm 15$ & $\ldots$ & $351 \pm 21^{s d}$ & $10.11 \pm 0.02^{\dagger}$ & $8312-8634$ \\
\hline 116 & $131 /-$ & 54.7 & 65 & 32.3 & 64.6 & 8.0 & W10a & $\ldots$ & $\ldots$ & $106 \pm 6^{n r}$ & W10a & $\ldots$ \\
\hline 117 & $-/ 46^{*}$ & 29.6 & $\ldots$ & 8.4 & $\ldots$ & $\ldots$ & $\ldots$ & $59 \pm 7$ & 24 & $83 \pm 10^{s d}$ & $<6.02$ & $607-703$ \\
\hline 118 & $1 * /-$ & 23.1 & 10 & 25.0 & 30.6 & 15.4 & W10a & $\ldots$ & $\ldots$ & {$[86 \pm 37]^{n r}$} & W10a & $\ldots$ \\
\hline 120 & $63 /-$ & 29.6 & [20] & 59.1 & 47.6 & 3.9 & W10a & $\ldots$ & $\ldots$ & {$[70 \pm 12]^{i r}$} & W10a & $\ldots$ \\
\hline 121 & $89 /-$ & 52.8 & 24 & 15.9 & 29.5 & 21.2 & W10a & $\ldots$ & $\ldots$ & $48 \pm 11^{i r}$ & W10a & $\ldots$ \\
\hline 122 & $18 /-$ & 39.4 & 31 & 24.3 & 39.7 & 3.1 & [W10a] & $\ldots$ & $\ldots$ & {$[71 \pm 13]^{i r}$} & $\mathrm{~W} 10 \mathrm{a}^{\ddagger}$ & $\ldots$ \\
\hline 123 & $40 /-$ & 78.9 & 105 & 51.6 & 93.0 & 5.1 & W10a & $\ldots$ & $\ldots$ & $115 \pm 10^{i r}$ & W10a & $\ldots$ \\
\hline 124 & $33 /-$ & 54.7 & 28 & 10.5 & 21.5 & 30.2 & [W10a] & $\ldots$ & $\ldots$ & $52 \pm 12^{i r}$ & W10 $\mathrm{a}^{\ddagger}$ & $\ldots$ \\
\hline 125 & $100 / 100$ & 45.6 & 99 & 11.7 & 21.5 & 6.0 & $242 \pm 4$ & $61 \pm 13^{1}$ & $\ldots$ & $169 \pm 49^{n r}$ & $9.97 \pm 0.02$ & $6680-7124$ \\
\hline 126 & $80 /-$ & 44.9 & 27 & 30.1 & 35.2 & 25.6 & W10a & $\ldots$ & $\ldots$ & $59 \pm 12^{i r}$ & W10a & $\ldots$ \\
\hline 127 & $159 /-$ & 79.6 & [86] & 47.6 & 57.8 & 6.9 & W10a & $\ldots$ & $\ldots$ & {$[97 \pm 10]^{i r}$} & W10a & $\ldots$ \\
\hline 128 & $115 / 116$ & 31.7 & 34 & 22.9 & 10.2 & 28.7 & [W10a] & $160 \pm 10^{1}$ & 178 & {$[374 \pm 64]^{s r}$} & $\mathrm{~W} 10 \mathrm{a}^{\ddagger}$ & $\ldots$ \\
\hline 129 & $-/ 30^{*}$ & 42.5 & $\ldots$ & 8.2 & $\ldots$ & $\ldots$ & $\ldots$ & $203 \pm 12$ & $\ldots$ & $286 \pm 17^{s d}$ & $<8.83$ & $6801-7176$ \\
\hline 130 & $-/ 135^{*}$ & 0.0 & $\ldots$ & 8.0 & $\ldots$ & $\ldots$ & $\ldots$ & $172 \pm 11$ & $\ldots$ & $243 \pm 15^{s d}$ & $<9.67^{\mathrm{k}}$ & $6170-6408$ \\
\hline 131 & $-/ 71^{*}$ & 25.2 & $\ldots$ & 13.1 & $\ldots$ & $\ldots$ & $\ldots$ & $266 \pm 15$ & $\ldots$ & $376 \pm 22^{s d}$ & $<8.77$ & $7710-8075$ \\
\hline 132 & $100 / 100$ & 47.8 & 105 & 17.1 & 36.3 & 2.7 & W10a & $49 \pm 12^{1}$ & $\ldots$ & $152 \pm 20^{i r}$ & W10a & $\ldots$ \\
\hline
\end{tabular}


Table 2

(Continued)

\begin{tabular}{|c|c|c|c|c|c|c|c|c|c|c|c|c|}
\hline ID & $\begin{array}{l}\text { P.A. }^{\text {a }} \\
\left({ }^{\circ}\right)\end{array}$ & $\begin{array}{l}i^{\mathrm{b}} \\
\left(^{\circ}\right)\end{array}$ & $\begin{array}{c}V_{\mathrm{pmm}^{\mathrm{c}}} \\
\left(\mathrm{km} \mathrm{s}^{-1}\right)\end{array}$ & $\begin{array}{l}r_{e}^{\mathrm{d}} \\
\left({ }^{\prime \prime}\right)\end{array}$ & $\begin{array}{c}\text { Extent } \\
\left({ }^{\prime \prime}\right)\end{array}$ & $\begin{array}{c}\text { Asym. } \\
(\%)\end{array}$ & $\begin{array}{c}\mathrm{W}_{50}{ }^{\mathrm{e}} \\
\left(\mathrm{km} \mathrm{s}^{-1}\right)\end{array}$ & $\begin{array}{c}\sigma_{r_{e} / 4} \\
\left(\mathrm{~km} \mathrm{~s}^{-1}\right)\end{array}$ & $\begin{array}{c}V_{*}^{\mathrm{f}} \\
\left(\mathrm{km} \mathrm{s}^{-1}\right)\end{array}$ & $\begin{array}{c}V^{\mathrm{g}} \\
\left(\mathrm{km} \mathrm{s}^{-1}\right)\end{array}$ & $\begin{array}{l}\log M_{\mathrm{H}_{\mathrm{I}}}{ }^{\mathrm{h}} \\
\left(\log M_{\odot}\right)\end{array}$ & $\begin{array}{l}\text { H I Rangej } \\
\left(\mathrm{km} \mathrm{s}^{-1}\right)\end{array}$ \\
\hline 133 & $80 /-$ & 75.8 & 85 & 23.3 & 35.2 & 5.7 & $208 \pm 2$ & $\cdots$ & $\ldots$ & $107 \pm 4^{n r}$ & $9.79 \pm 0.01$ & $2340-2760$ \\
\hline 134 & $172 /-$ & 90.0 & 88 & 35.7 & 57.8 & 4.8 & W10a & $\cdots$ & $\ldots$ & $98 \pm 10^{i r}$ & W10a & $\ldots$ \\
\hline 135 & $40 /-$ & 29.6 & 65 & 11.1 & 19.3 & 7.4 & $156 \pm 2$ & $\cdots$ & $\cdots$ & {$[158 \pm 84]^{n r}$} & $9.39 \pm 0.02$ & 3179-3596 \\
\hline 136 & $153 /-$ & 65.4 & 101 & 27.4 & 53.3 & 4.9 & W10a & $\ldots$ & $\ldots$ & $121 \pm 11^{i r}$ & W10a & $\ldots$ \\
\hline 137 & $100^{*} / 100^{*}$ & 39.7 & [82] & 9.4 & 6.8 & $\cdots$ & W10a & $101 \pm 8$ & $\ldots$ & $143 \pm 12^{s d}$ & W10a & $\cdots$ \\
\hline 138 & $1 / 170 *$ & 75.1 & 285 & 26.3 & 55.6 & 4.9 & W10a & $196 \pm 12^{1}$ & $\ldots$ & $309 \pm 24^{n r}$ & W10a & $\ldots$ \\
\hline 139 & $-/ 170$ & 71.1 & $\ldots$ & 18.0 & $\ldots$ & $\ldots$ & {$[402 \pm 10]$} & $257 \pm 15$ & $\ldots$ & $363 \pm 22^{s d}$ & $9.50 \pm 0.05^{\ddagger \mathrm{k}}$ & $\ldots$ \\
\hline 140 & $1 * /-$ & 31.7 & 23 & 28.1 & 46.5 & 9.9 & W10a & $\ldots$ & $\ldots$ & {$[141 \pm 23]^{n r}$} & W10a & $\cdots$ \\
\hline 141 & $100 /-$ & 77.3 & 69 & 27.4 & 31.8 & 4.0 & W10a & $\ldots$ & $\ldots$ & $95 \pm 5^{n r}$ & W10a & $\ldots$ \\
\hline 142 & $60 / 56$ & 72.4 & 290 & 21.7 & 32.9 & 1.4 & W10a & $175 \pm 12^{1}$ & 249 & $298 \pm 17^{i r}$ & W10a & $\ldots$ \\
\hline 143 & $168 /-$ & 76.2 & 114 & 20.3 & 30.6 & 3.2 & W10a & $\ldots$ & $\ldots$ & $125 \pm 11^{i r}$ & W10a & $\ldots$ \\
\hline 144 & $100 / 100$ & 57.3 & {$[21]$} & 22.9 & 4.5 & 11.5 & $46 \pm 16^{\dagger \dagger}$ & $45 \pm 11^{1}$ & $\ldots$ & {$[64 \pm 16]^{s d}$} & $7.50 \pm 0.06$ & $668-925$ \\
\hline 145 & $18 / 16$ & 79.6 & [139] & 52.9 & 62.4 & 3.5 & W10a & $34 \pm 9^{1}$ & $\ldots$ & {$[146 \pm 10]^{i r}$} & W10a & $\ldots$ \\
\hline 146 & $-/ 30$ & 78.7 & $\ldots$ & 10.9 & $\ldots$ & $\ldots$ & $\ldots$ & $142 \pm 10$ & 153 & $201 \pm 14^{s d}$ & $<7.94$ & $1900-2282$ \\
\hline 147 & $-/ 100$ & 45.6 & $\ldots$ & 19.1 & $\cdots$ & $\cdots$ & $\ldots$ & $194 \pm 12$ & $\ldots$ & $275 \pm 16^{s d}$ & $<8.50$ & $5230-5610$ \\
\hline 148 & $127 /-$ & 82.6 & 109 & 28.1 & 48.8 & 1.4 & W10a & $\ldots$ & $\ldots$ & $118 \pm 10^{i r}$ & W10a & $\ldots$ \\
\hline 149 & $40 / 36$ & 83.3 & $\ldots$ & 14.7 & $\ldots$ & $\ldots$ & $\ldots$ & $151 \pm 10$ & 164 & $213 \pm 14^{s d}$ & $<8.39$ & $3632-4040$ \\
\hline 150 & $65 / 56$ & 90.0 & [120] & 46.2 & 59.0 & 2.1 & W10a & $30 \pm 15^{1}$ & $\ldots$ & {$[127 \pm 10]^{i r}$} & W10a & $\ldots$ \\
\hline 151 & $80 /-$ & 52.8 & 227 & 17.1 & 44.2 & 1.9 & [W10a] & $\ldots$ & $\ldots$ & $283 \pm 28^{i r}$ & $\mathrm{~W} 10 \mathrm{a}^{\ddagger}$ & $\ldots$ \\
\hline 152 & $-/ 140$ & 0.0 & $\ldots$ & 22.3 & $\ldots$ & $\ldots$ & $\ldots$ & $341 \pm 19$ & $\ldots$ & $483 \pm 27^{s d}$ & $<9.10$ & $7214-7676$ \\
\hline 153 & $1 /-$ & 39.9 & 156 & 10.3 & 18.1 & 2.0 & {$[339 \pm 13]$} & $\ldots$ & $\ldots$ & {$[250 \pm 71]^{i r}$} & $10.19 \pm 0.02^{\dagger}$ & $7540-7860$ \\
\hline 154 & $-/ 100$ & 42.5 & $\ldots$ & 9.4 & $\ldots$ & $\ldots$ & {$[71 \pm 143]$} & $113 \pm 10$ & $\ldots$ & $160 \pm 14^{s d}$ & $8.80 \pm 0.11$ & $2934-3588$ \\
\hline 155 & $100 /-$ & 57.2 & 41 & 33.2 & 42.0 & 8.1 & W10a & $\ldots$ & $\ldots$ & $64 \pm 10^{i r}$ & W10a & $\ldots$ \\
\hline 156 & $100 / 100$ & 62.1 & 98 & 16.3 & 31.8 & 2.9 & W10a & $\ldots$ & $\ldots$ & $129 \pm 9^{n r}$ & W10a & $\ldots$ \\
\hline 157 & $-/ 100$ & 33.4 & $\cdots$ & 19.8 & $\cdots$ & $\cdots$ & $\ldots$ & $164 \pm 11$ & $\ldots$ & $231 \pm 16^{s d}$ & $<8.24$ & $3981-4234$ \\
\hline 158 & $140 / 140$ & 42.5 & 80 & 19.2 & 47.6 & 2.8 & W10a & $75 \pm 7^{1}$ & $\ldots$ & $134 \pm 18^{i r}$ & W10a & $\ldots$ \\
\hline 159 & 68/- & 75.8 & 28 & 7.4 & 22.7 & 47.4 & W10a & $\ldots$ & $\cdots$ & $93 \pm 6^{n r}$ & W10a & $\cdots$ \\
\hline 160 & $29 /-$ & $68.0(0.0)$ & 61 & 10.7 & 22.7 & 16.4 & W10a & $\ldots$ & $\ldots$ & $92 \pm 7^{n r}$ & W10a & $\cdots$ \\
\hline 161 & $68 / 75$ & 56.9 & 93 & 17.5 & 34.0 & 3.4 & [W10a] & $44 \pm 7^{1}$ & $\ldots$ & $122 \pm 15^{i r}$ & $\mathrm{~W} 10 \mathrm{a}^{\ddagger}$ & $\ldots$ \\
\hline 162 & $80 /-$ & 66.6 & 19 & 11.6 & 17.0 & 10.6 & W10a & $\ldots$ & $\ldots$ & $38 \pm 3^{n r}$ & W10a & $\ldots$ \\
\hline 164 & $60 /-$ & 46.8 & 110 & 12.7 & 22.7 & 3.7 & $279 \pm 7$ & $\ldots$ & $\ldots$ & $192 \pm 37^{n r}$ & $9.98 \pm 0.02$ & $6240-7040$ \\
\hline 165 & $55 / 54$ & 50.6 & [119] & 35.4 & 45.4 & 2.5 & W10a & $75 \pm 8^{1}$ & $\ldots$ & $195 \pm 14^{n r}$ & W10a & $\ldots$ \\
\hline 166 & 100/- & 27.9 & 22 & 10.6 & 17.0 & 9.8 & $123 \pm 5$ & $\ldots$ & $\ldots$ & {$[132 \pm 66]^{n r}$} & $8.76 \pm 0.02$ & $2310-2650$ \\
\hline 167 & $159 / 140 *$ & 54.7 & 256 & 18.5 & 26.1 & 1.9 & $544 \pm 6$ & $181 \pm 12^{1}$ & $\ldots$ & $333 \pm 31^{n r}$ & $9.97 \pm 0.03$ & $8400-9100$ \\
\hline 168 & $80 / 75$ & 66.1 & 180 & 11.8 & 29.5 & 1.6 & W10a & $100 \pm 10^{1}$ & 157 & $199 \pm 21^{i r}$ & W10a & $\ldots$ \\
\hline 170 & $12 /-$ & 42.5 & 92 & 17.4 & 34.0 & 3.0 & W10a & $\ldots$ & $\ldots$ & $187 \pm 27^{n r}$ & W10a & $\ldots$ \\
\hline 171 & $140 / 140$ & $32.0(42.5)$ & [71] & 13.2 & 15.9 & 17.9 & W10a & $\ldots$ & $\ldots$ & {$[161 \pm 40]^{n r}$} & W10a & $\ldots$ \\
\hline 172 & $94 / 100$ & 26.4 & 44 & 6.0 & 18.1 & 11.2 & $194 \pm 5$ & $98 \pm 9$ & $\ldots$ & $139 \pm 13^{s d}$ & $9.52 \pm 0.02$ & $4249-4524$ \\
\hline 173 & $-/ 100$ & 45.6 & $\ldots$ & 6.9 & $\ldots$ & $\ldots$ & $\ldots$ & $205 \pm 14$ & $\ldots$ & $290 \pm 20^{s d}$ & $<9.07$ & 11987-12387 \\
\hline 174 & $66 / 66$ & 46.3 & 173 & 20.2 & 45.4 & 3.5 & W10a & $133 \pm 10^{1}$ & $\ldots$ & $270 \pm 31^{n r}$ & W10a & $\ldots$ \\
\hline 175 & $177 / 178$ & 78.0 & 101 & 10.9 & 15.9 & 2.4 & $\ldots$ & $34 \pm 13^{1}$ & $\ldots$ & $112 \pm 12^{i r}$ & $<8.40^{\mathrm{k}}$ & $2115-2354$ \\
\hline 176 & $-/ 140$ & 33.0 & $\ldots$ & 16.7 & $\ldots$ & $\ldots$ & {$[113 \pm 15]$} & $314 \pm 18$ & $\ldots$ & $444 \pm 26^{s d}$ & $8.77 \pm 0.08^{\dagger}$ & $5880-6088$ \\
\hline 177 & $140 / 140$ & 45.6 & $\ldots$ & 17.1 & $\ldots$ & $\ldots$ & W10a & $189 \pm 11$ & $\ldots$ & $267 \pm 16^{s d}$ & W10a & $\ldots$ \\
\hline 178 & $-/ 2$ & 81.2 & $\ldots$ & 10.8 & $\ldots$ & $\ldots$ & W10a & $134 \pm 9$ & 182 & $203 \pm 19^{s r}$ & W10a & $\ldots$ \\
\hline 179 & $140 * /-$ & 0.0 & 99 & 20.1 & 30.6 & 3.5 & W10a & $\ldots$ & $\ldots$ & $\ldots$ & W10a & $\ldots$ \\
\hline 180 & $1 / 2$ & 49.5 & [238] & 19.0 & 7.9 & 3.8 & $\ldots$ & $236 \pm 14^{1}$ & 163 & {$[333 \pm 20]^{s d}$} & $<9.47$ & 10039-10631 \\
\hline 181 & $44 * / 45^{*}$ & $40.0(0.0)$ & 32 & 7.0 & 10.2 & 5.5 & [W10a] & $38 \pm 14^{1}$ & $\ldots$ & {$[71 \pm 11]^{i r}$} & $\mathrm{~W} 10 \mathrm{a}^{\ddagger}$ & $\ldots$ \\
\hline 182 & $-/ 20$ & 47.4 & $\ldots$ & 18.8 & $\ldots$ & $\ldots$ & [W10a] & $268 \pm 15$ & $\ldots$ & $379 \pm 22^{s d}$ & $\mathrm{~W} 10 \mathrm{a}^{\ddagger}$ & $\ldots$ \\
\hline 183 & $145 / 145.1$ & 49.5 & 79 & 9.9 & 15.9 & 2.8 & W10a & $28 \pm 7^{1}$ & 68 & $117 \pm 36^{i r}$ & $\mathrm{~W} 10 \mathrm{a}$ & $\ldots$ \\
\hline 184 & $88 / 90$ & 74.2 & 151 & 24.8 & 53.3 & 2.9 & W10a & $82 \pm 10^{1}$ & 146 & $171 \pm 18^{s r}$ & W10a & $\ldots$ \\
\hline 185 & $153 / 153$ & 67.2 & 167 & 12.6 & 21.5 & 4.9 & W10a & $124 \pm 9$ & $\ldots$ & $184 \pm 21^{i r}$ & W10a & $\ldots$ \\
\hline 186 & 10/- & 61.0 & 110 & 50.6 & 73.7 & 9.1 & W10a & $\ldots$ & $\ldots$ & $134 \pm 11^{i r}$ & W10a & $\ldots$ \\
\hline 187 & $90 / 90$ & $57.0(42.5)$ & 101 & 5.5 & 7.9 & 2.8 & {$[196 \pm 7]$} & $78 \pm 13^{1}$ & 104 & $145 \pm 19^{s r}$ & $9.65 \pm 0.03^{\dagger}$ & $5605-5846$ \\
\hline 188 & $-/ 90 *$ & 0.0 & $\ldots$ & 28.1 & $\ldots$ & $\ldots$ & $\ldots$ & $275 \pm 16$ & $\ldots$ & $389 \pm 22^{s d}$ & $<9.42^{\mathrm{k}}$ & $7233-7611$ \\
\hline 189 & $45 / 45$ & 25.2 & 91 & 17.9 & 30.6 & 2.2 & W10a & $45 \pm 12^{1}$ & $\ldots$ & {$[236 \pm 109]^{i r}$} & W10a & $\ldots$ \\
\hline 190 & $79 / 90 *$ & 82.4 & 146 & 24.4 & 62.4 & 3.1 & W10a & $66 \pm 9^{1}$ & $\ldots$ & $155 \pm 5^{n r}$ & W10a & $\ldots$ \\
\hline 191 & $90 * / 90 *$ & 37.8 & 74 & 15.0 & 22.7 & 13.8 & W10a & $182 \pm 12$ & $\ldots$ & $258 \pm 16^{s d}$ & W10a & $\ldots$ \\
\hline 192 & $158 / 158.1$ & 78.0 & 153 & 22.4 & 38.6 & 1.6 & W10a & $70 \pm 7^{1}$ & 154 & $177 \pm 18^{s r}$ & W10a & $\ldots$ \\
\hline 193 & $158^{*} / 158^{*}$ & 23.1 & 88 & 10.5 & 21.5 & 3.4 & W10a & $85 \pm 9^{1}$ & $\ldots$ & {$[303 \pm 129]^{n r}$} & W10a & $\ldots$ \\
\hline 194 & $-/ 90 *$ & 31.7 & $\ldots$ & 18.4 & $\ldots$ & $\ldots$ & W10a & $297 \pm 17$ & $\ldots$ & $420 \pm 25^{s d}$ & W10a & $\ldots$ \\
\hline 195 & $94 / 94$ & 79.9 & 216 & 28.3 & 49.9 & 5.7 & W10a & $127 \pm 13^{1}$ & $\ldots$ & $219 \pm 11^{i r}$ & W10a & $\ldots$ \\
\hline
\end{tabular}


Table 2

(Continued)

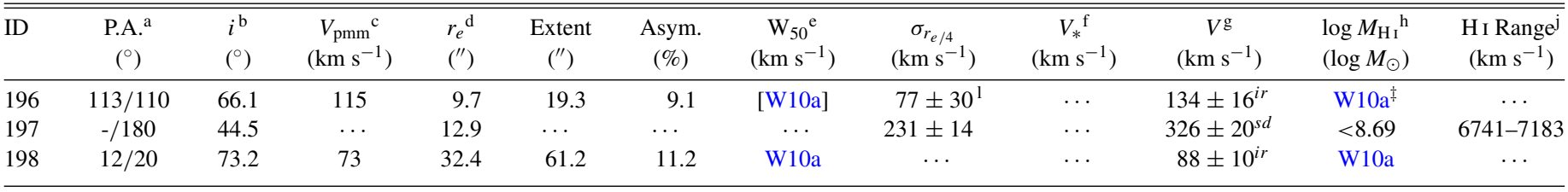

Notes.

${ }^{\text {a }}$ Slit P.A.s of observations used to derive ionized gas rotation curve and stellar absorption line kinematics, respectively.

${ }^{\mathrm{b}}$ Parentheses indicate inclination estimates from KFF, usually inferior except for UGC 9562 and NGC 3499, for which the new estimate applies only to the gas, not the stars.

${ }^{\mathrm{c}}$ Bracketed numbers denote unreliable velocities (Section 2.1.3). Uncertainties on $V_{\mathrm{pmm}}$ are set to $11 \mathrm{~km} \mathrm{~s}^{-1}$ following KFF (Section 2.1.3).

${ }^{\mathrm{d}}$ Half-light radius in the $B$ band from Jansen et al. (2000b), converted from the authors' geometric mean aperture radius convention to a major axis radius convention. This $B$-band radius is denoted $r_{e}$ as distinct from the $r$-band half-light radius denoted $r_{50}^{r}$ in Table 1 .

${ }^{\mathrm{e}}$ Bracketed numbers denote unreliable linewidths (Section 2.1.3).

${ }^{\mathrm{f}}$ Uncertainties in $V_{*}$ are set to $17 \mathrm{~km} \mathrm{~s}^{-1}$ (Section 2.1.3).

$\mathrm{g}$ Bracketed numbers denote unreliable linewidths.

${ }^{\mathrm{h}}$ Mass includes factor of 1.4 to account for He.

${ }^{\mathrm{j}}$ Range of heliocentric velocities used in the $\mathrm{H}$ I flux measurement or upper limit determination.

${ }^{\mathrm{k}} \mathrm{H}$ i data from ALFALFA survey, with upper limits estimated from the survey sensitivity limit (Haynes et al. 2011).

${ }^{1}$ Stellar velocity dispersion may be an unreliable metric of characteristic velocity $V$, based on either late-type morphology or low dispersion (Section 2.1.3).

* P.A. uncertain or slit P.A. misaligned by more than $10^{\circ}$ with the galaxy major axis.

$\dagger$ Confirmed likely confused; H I flux corrected as described in Section 2.1.2 and H I linewidth designated as unreliable.

$\ddagger$ Literature H I data possibly subject to confusion based on identification of a close companion or ongoing interaction/merger by KFF.

$\dagger^{\dagger}$ Linewidth inconsistent with much larger ALFALFA value; both come from low-S/N ( 6) spectra and are inconsistent with the Tully-Fisher relation.
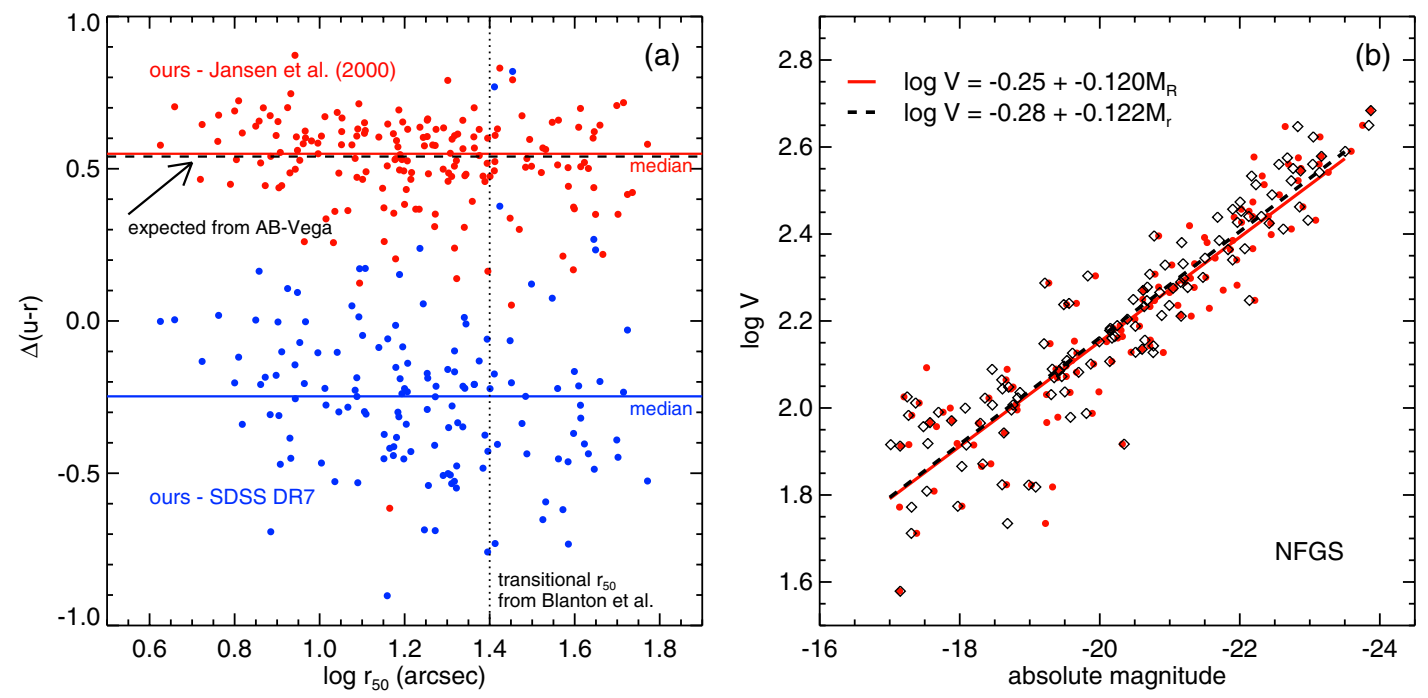

Figure 2. Comparisons of photometric and kinematic data for the NFGS. (a) Our newly measured $u-r$ colors compared to catalog $u-r$ colors from SDSS DR7 (Abazajian et al. 2009) and $U-R$ colors from Jansen et al. (2000b), as a function of $R$-band half-light radius (converted from the $\sqrt{a b}$ convention of Jansen et al. to a major-axis convention). Our colors are in excellent agreement with those of Jansen et al. after allowing for the known 0.04 mag AB offset of SDSS $u$ and the 0.58 mag expected offset from Vega-to-AB conversions ( $m_{\text {Vega, } \mathrm{AB}}$ of 0.79 and 0.21 for $U$ and $R$, respectively; see Blanton \& Roweis 2007). The disagreement with SDSS catalog colors likely reflects (1) our use of the improved sky subtraction of Blanton et al. (2011), which those authors find to give bluer colors for log $r_{50} \gtrsim 1.4$; (2) the existence of color gradients, which are included in our photometry and that of Jansen et al. but forcibly set to zero by the SDSS model magnitude algorithm; and (3) catastrophic errors in the SDSS pipeline, contributing to the large scatter, including five extreme outliers outside the plot bounds, all with blue offsets $\Delta(u-r)<-1$. (b) Characteristic velocity $V$ (rotation speed or $\sqrt{2} \times$ stellar dispersion $\sigma$; see Section 2.1.3) vs. $M_{r}$ and $M_{R}$. Lines show forward fits minimizing residuals in $V$ with coefficients as indicated. The close coincidence of zero points is spurious, reflecting the canceling effects of the 0.21 mag Vega-to-AB conversion for $R$ and the $\sim 0.2$ mag across-the-board zero-point difference between the Jansen et al. UBR photometry and our own (see footnote 17). Both $M_{r}$ and $M_{R}$ include foreground extinction and $k$-corrections but omit internal extinction corrections.

(A color version of this figure is available in the online journal.)

fits both the spectral energy distribution (SED) and the integrated spectrum (if available) of a galaxy with a suite of composite stellar population models. The code combines old+young simple stellar populations (SSPs) from Bruzual \& Charlot (2003) in a grid of models with varying age, metallicity, and percentage of young stars. The models allow for 11 different extinction/ reddening values $\left(\tau_{V}=0,0.12,0.24, \ldots, 1.2\right)$ applied to the young SSP using the Calzetti (2001) dust law. Likelihoods and stellar masses are computed for all models in the grid, and the median of the likelihood-weighted stellar mass distribution provides the most robust final stellar mass estimate, with uncertainties determined from the $68 \%$ confidence interval around the median.

In the present work we consider two different model grids. The first, also used by S13, assumes a "diet" Salpeter initial mass function (IMF; Bell et al. 2003). It combines an old SSP 
(age $1.4,2.5,3.5,4.5, \ldots, 13.5 \mathrm{Gyr}$ ) with a young SSP (age $5,25,100,290$, or $1000 \mathrm{Myr}$ ), with the younger component contributing $0 \%, 1 \%, 2 \%, 4 \%, 8 \%, 16 \%, 32 \%$, or $64 \%$ of the mass. This grid differs from that of KGB only in adding a young population age of $5 \mathrm{Myr}$. We note also that although not stated explicitly in KGB, the code does allow a "middleaged" young population, i.e., one with any of the "old" ages younger than the designated old population age (for example, 2.5 Gyr combined with $13.5 \mathrm{Gyr}$ ). We compensate for the overrepresentation of $\lesssim 1$ Gyr SSPs in old+young pairings by downweighting the likelihoods for these pairings such that all $\lesssim 1$ Gyr SSPs together have equal weight to one "middleaged" population option, approximating a uniform prior on the age of the younger component. Following KGB, this model set includes three metallicities $Z=0.008,0.02$, and 0.05 (solar/2.5, solar, and solar $\times 2.5)$.

The second model grid is our primary one for the present work. It is designed to enable estimation of the ratio of stellar mass formed within the last Gyr to preexisting stellar mass (where this ratio equals the long-term fractional stellar mass growth rate, $\mathrm{FSMGR}_{\mathrm{LT}}$ ) and therefore includes both single-burst and continuous SFH options for the young population. To facilitate comparison with the work of Salim et al. (2007, hereafter S07) in Section 4.2, our model grid emulates theirs in including an additional low-metallicity choice $(Z=0.004)$ and adopting a Chabrier IMF. We further consider that even continuous star formation can be bursty on 200 Myr timescales (e.g., Weisz et al. 2012), which S07 choose to model with superposition of random bursts. To keep the grid size manageable, since only the most recent bursts are likely to strongly affect the model fits, we take the alternative approach of constructing a set of young population models that have constant star formation running from $1015 \mathrm{Myr}$ ago to a turn-off point sometime between 0 and $195 \mathrm{Myr}$ ago, sampled every 15 Myr. The young population options also include 5 models representing quenching bursts without subsequent star formation: SSPs with ages 360, 509, 641, 806, and 1015 Myr. For computational convenience, we restrict the old SSP choices in this model grid to six ages (2, 4, 6, 8, 10, and 12 Gyr). However, we now consider 13 young stellar population mass fractions $(0.001,0.002,0.005,0.011,0.025,0.053,0.112,0.220$, $0.387,0.585,0.760,0.876,0.941)$, equally spaced logarithmically in FSMGR $\mathrm{LT}_{\mathrm{LT}}$. Once again, an old SSP can serve as the younger model in an old+young pair, and FSMGR $\mathrm{FT}_{\mathrm{LT}}=0$ in such a case. The overrepresentation of pairings with young age $\lesssim 1 \mathrm{Gyr}$ is approximately canceled by downweighting the likelihoods for these models, where we treat all of the continuous SFH models together as having combined weight equal to one of the five $\lesssim 1$ Gyr bursts and then further downweight both types of young models relative to the "middle-aged" SSPs to approximate a uniform prior on the age of the younger component.

As data are available, we simultaneously fit these model grids to NUV+ugrizJHK+IRAC $3.6 \mu \mathrm{m}$ SEDs as well as optical spectroscopy. We apply the $0.04 \mathrm{mag} \mathrm{AB}$ offset for the $u$ band $^{17}$ inside the code. To the individual magnitude errors we add extra photometric uncertainties to account for variations between methods of foreground extinction correction and sky level estimation: $0.1 \mathrm{mag}$ in the NUV, $0.05 \mathrm{mag}$ in $u, 0.03 \mathrm{mag}$ in griz, and $0.1 \mathrm{mag}$ in $J H K+\mathrm{IRAC} 3.6 \mu \mathrm{m}$, with an extra $0.1 \mathrm{mag}$ in $J H K$ for faint blue galaxies $\left(M_{r}\right.$ or $M_{R}>-19$ and $u-r<1.4$ or $U-R<0.7)$. These choices are motivated by the analyses

\footnotetext{
17 http://www.sdss.org/dr7/algorithms/fluxcal.html\#sdss2ab
}

of Abazajian et al. (2004), Morrissey et al. (2007), and Blanton et al. (2011), as well as our own analysis of the agreement of our JHK magnitudes with the best fits. When SDSS data are unavailable, we substitute the $U B R$ magnitudes from Jansen et al. (2000b), applying a uniform 0.2 mag offset to reconcile the $U B R$ zero points with our brighter SDSS magnitudes, where this offset value is estimated by fitting both ugriz and $U B R$ simultaneously when possible in an initial round of SED fits. Our final fits do not include $U B R$ when we have ugriz, however, to minimize systematics. We note that our estimated acrossthe-board $\sim 0.2 \mathrm{mag}$ offset is opposite to the Vega-to-AB offset for the $R$ band and thus yields fortuitous zero-point agreement between the $r$ and $R$ bands (as seen, for example, by comparing the merged NFGS Tully-Fisher/Faber-Jackson relations shown in Figure 2; see Section 2.1.3). ${ }^{18}$

Stellar masses estimated with the second model grid are provided in Table 1. With so many independent data points, these mass estimates are quite robust; estimates derived using the first model grid are offset 0.1 dex higher than those derived using the second model grid but otherwise agree within $0.1 \mathrm{dex}$ rms (less than the typical uncertainty of 0.15 dex from the stellar mass distributions). The only obviously unreliable case is UGC 4879 , an extremely nearby system for which our photometry is notably inconsistent, possibly due to the technical difficulty of sky subtraction or to a complex post-starburst SED (the same galaxy lacks any emission or absorption features and thus also defies kinematic analysis). For reference, Table 1 also provides the stellar masses previously derived by KGB using a model set very similar to our first model grid, but with inferior photometry (uncorrected $U B R+$ catalog $2 \mathrm{MASS}$ data); these masses show 0.2 dex scatter and +0.06 dex median offset relative to our preferred masses. We include the KGB masses because they have been used in several recent papers (Wei et al. 2010a, 2010b; Moffett et al. 2012) and compared to stellar masses from Kauffmann et al. (2003b); this comparison demonstrates similar stellar-mass zero points (Kannappan \& Wei 2008).

To ensure uniform color data, we use our stellar population model fits to interpolate likelihood-weighted $(u-r)^{m}$ and $(u-J)^{m}$ colors for all galaxies, regardless of the availability of these specific bands, where the superscript $m$ is a reminder that these colors come from the models and thus include the $\mathrm{AB}$ correction to the $u$ band as well as $k$-corrections to $z=0$. Selfconsistent internal extinction corrections can also be determined with our newer model grid, enabling us to examine the behavior of the de-extincted colors, denoted $(u-r)^{e}$ and $(u-J)^{e}$.

Half-light and $90 \%$ light radii are also given in Table 1 , as these radii are used to compute $\mu_{\Delta}$, a new quantitative morphology metric introduced in this work to facilitate comparison of the NFGS and V3000 samples. We define $\mu_{\Delta}$ as

$$
\mu_{\Delta}=\mu_{90}+1.7 \Delta \mu
$$

\footnotetext{
18 Applying the sky subtraction technique described in Jansen et al. (2000b) to SDSS DR8 images (which are pre-sky-subtracted using the methods of Blanton et al. 2011) reveals that $\sim 0.1$ mag of the offset we measure is probably due to oversubtraction of sky by Jansen et al. compared to the new protocol (as expected from Figure 12 of Blanton et al.). The remainder may be partly due to a mismatch between the $U B R$ filter systems used in our stellar population fitting code and used by Jansen et al., and/or partly due to differing profile extrapolation techniques. Regarding the latter, we note that the zero-point discrepancy increases for low surface brightness galaxies, in the sense that our reprocessed SDSS magnitudes are brighter and the spurious agreement between the $V-M_{r} / M_{R}$ relations in Figure 2 becomes tighter.
} 
combining an overall surface mass density

$$
\mu_{90}=\log \frac{0.9 M_{*}}{\pi r_{90, r}^{2}}
$$

with a surface mass density contrast

$$
\Delta \mu=\log \frac{0.5 M_{*}}{\pi r_{50, r}^{2}}-\log \frac{0.4 M_{*}}{\pi r_{90, r}^{2}-\pi r_{50, r}^{2}}
$$

representing the difference between the surface mass densities within the $50 \%$ light radius and between the $50 \%$ and $90 \%$ light radii, where all radii are converted to physical kiloparsec units. The 1.7 multiplier helps to separate quasi-bulgeless, bulge+disk, and spheroid-dominated types as illustrated in Figure 1(b), yielding approximate divisions at $\mu_{\Delta}=8.6$ and 9.5 as shown. For galaxies without $r_{50, r}$ measurements, we use the $R$-band half-light radii from Jansen et al. (2000b), converted from the authors' geometric mean aperture radius convention to a major axis radius convention. We find a one-to-one correspondence between the $r$ - and $R$-band half-light radii, with rms scatter $\lesssim 15 \%$ and a small $3.3 \%$ offset, in the sense of larger $r$-band radii. When using $r_{50, R}$ we assume the median value of $r_{90, r} / r_{50, r}=$ 2.6 to infer $r_{90, R}$.

\subsubsection{Gas Masses and H I Linewidths}

The Hi data set presented in Table 2 expands on that of Wei et al. (2010a, hereafter W10a) with 30 new Robert C. Byrd Green Bank Telescope (GBT) $21 \mathrm{~cm}$ observations taken for the NFGS under program GBT10A-070 in 2010 January, February, and July (PI: Kannappan). We add these to the 27 GBT observations obtained by W10a under programs GBT07A072 and GBT07C-148 in 2007 March and October. We have reprocessed the $\mathrm{W} 10 \mathrm{a}$ data along with our new data since discovering that the default GBTIDL flux calibration, which was used by W10a, is 15\%-20\% lower than that obtained from the observed flux calibrators, and also since finding an error in how W10a estimated rms noise during linewidth measurement, which led to overestimation of linewidths by $\gtrsim 10 \%$. Other than these adjustments, our flux and linewidth measurements follow the methods of W10a closely for unconfused detections. In particular, we do not correct for self-absorption, which is expected to alter total $\mathrm{H}$ I flux estimates by $<30 \%$, even for the most inclined systems (Giovanelli et al. 1994). Note that the masses denoted $M_{\mathrm{H}}$ in Table 2 and the rest of this paper are measured directly from $21 \mathrm{~cm}$ fluxes using the equation $M_{\mathrm{HI}}=1.4 \times 2.36 \times 10^{5} f_{\mathrm{HI}}\left(c z / H_{0}\right)^{2} M_{\odot}$, which combines the expression from Haynes \& Giovanelli (1984) with a $1.4 \times$ correction factor for $\mathrm{He}$.

For non-detections and/or galaxies confused with companions in the beam, we have adopted a slightly different approach than W10a in our definition of integration bounds for estimating $3 \sigma$ upper limits and for dividing flux between objects. Upper limits are now calculated using the equivalent $W_{20}$ linewidths determined either from (a) the optically derived $V$, where $V$ is based on $\mathrm{H} \alpha$ or stellar rotation curves extending beyond $1.3 r_{e}$ (see Sections 2.1.3 and 2.1.4), or, in the absence of such data, from (b) the implied $V$ from the $R$-band absolute magnitude- $V$ relation $\log V=-0.25-0.120 M_{R}$, which has been calibrated using all galaxies with reliable $V$ and $M_{R}<-17$ in the NFGS (Figure 2). Here $V$ is estimated from either stellar or ionized gas kinematics as described in Sections 2.1.3 and 2.1.4 and is defined to scale as $W_{50} /(2 \sin i$ ) (so we adjust for an assumed offset of $W_{20}-W_{50}=20 \mathrm{~km} \mathrm{~s}^{-1}$; see Kannappan et al. 2002). We also account for the projection factor $\sin i$, adopting a minimum $i$ of $30^{\circ}$ for E galaxies to avoid overly strong upper limits, given the lack of reliable inclination information for such round objects. Likewise, we avoid overly strong upper limits for faceon disks by employing a minimum final integration linewidth of $40 \mathrm{~km} \mathrm{~s}^{-1}$ reflecting non-rotational line broadening. These definitions ensure that we compute conservative upper limits.

We have confirmed 11 cases of profiles confused with companion galaxies in our GBT data from a thorough search within twice the GBT half-power beam diameter of 9', assuming typical redshift uncertainties and linewidths and inspecting each of the 15 potential cases by eye. To isolate the flux for the primary target, we typically assign the primary all flux within the equivalent $W_{50}$ linewidth derived from the measured or inferred $V$ as discussed above, omitting the offset to the $W_{20}$ scale. Alternatively, if one half of the profile is obviously more contaminated, we integrate the uncontaminated half and double that flux. In the case of UGC $12265 \mathrm{~N}$, which is strongly interacting with a similar size companion and thus more severely confused than usual, W10a employ a Very Large Array $21 \mathrm{~cm}$ map to determine that only $\sim 25 \%$ of the flux belongs to the target galaxy, and we retain this flux division. Our flux separations are validated by the absence of significant outliers in our analysis (see especially Section 4.1).

Table 2 summarizes the final derived GBT $M_{\mathrm{H}_{\mathrm{I}}}$ values/ uncertainties or $3 \sigma$ upper limits, plus linewidths and velocity integration ranges. For galaxies that share the beam with one or more companions, linewidths represent the full $\mathrm{H}$ i profiles not deblended to account for confusion. Confused linewidths are thus enclosed in brackets to indicate that they are unreliable. Likewise, linewidths derived from profiles with peak $\mathrm{S} / \mathrm{N}<$ 6 are bracketed. Linewidth uncertainties are estimated using $\sigma_{W_{50}}=4.1(P /(\mathrm{S} / \mathrm{N}))^{0.85}$, where $P$ is the steepness parameter defined as $P=\left(W_{50}-W_{20}\right) / 2$ (this formula is derived as in $\mathrm{S} 13$ but assuming $5 \mathrm{~km} \mathrm{~s}^{-1}$ spectral resolution; see $\mathrm{S} 13$ for further details).

We have further augmented the $21 \mathrm{~cm}$ inventory for the NFGS using the literature compilation of W10a (not duplicated in Table 2) and the ALFALFA survey (Haynes et al. 2011). In particular, one detection and four upper limits inferred from ALFALFA are given in Table 2, with the upper limits estimated using the median rms noise as a function of declination (typically 2.3 mJy; Haynes et al. 2011). Unlike our GBT upper limits, ALFALFA upper limits are determined at $5 \sigma$, matching the survey detection threshold. The literature compilation of W10a includes the remaining 128 galaxies, so together, these data sets yield $M_{\mathrm{HI}}$ values or upper limits for all 190 galaxies in the sample, with only 1 of the 26 upper limits weaker than $10 \%$ of that galaxy's stellar mass (Section 2.1.1). As it is infeasible to uniformly assess and decompose confusion in the literature $\mathrm{H}$ i measurements, we have simply flagged likely cases of confusion in literature data based on the presence of a companion, interaction, or merger identified by Kannappan et al. (2002); such cases are marked in Table 2.

Molecular gas data are not uniformly available for the NFGS, but we have made use of $39 \mathrm{CO}$-derived $\mathrm{H}_{2}$ masses tabulated in Wei et al. (2010b) and/or in S13 to determine that including molecular gas in the total gas inventory has negligible impact on our conclusions except to reinforce them. The effect of molecular gas is illustrated in several figures in Sections 3 and 4, always with a $1.4 \times$ correction factor for $\mathrm{He}$. 


\subsubsection{Optical Kinematics}

Ionized gas and stellar rotation velocities and stellar velocity dispersions for the NFGS are also given in Table 2. These measurements are based on emission- and absorption-line observations previously reported in Kannappan et al. (2002, hereafter KFF) and Kannappan \& Fabricant (2001), coming from either the FAST Spectrograph on the Tillinghast telescope or the Blue Channel Spectrograph on the MMT telescope. For stellar dispersions $\sigma$, the instrumental resolution is at least $\sigma_{\text {instr }} \sim 60 \mathrm{~km} \mathrm{~s}^{-1}$ at the $\mathrm{Mg}$ I triplet near $5175 \AA$, improving to $\sim 40 \mathrm{~km} \mathrm{~s}^{-1}$ for the MMT data. We tabulate the stellar dispersion $\sigma_{r_{e} / 4}$ measured within one-fourth the $B$-band half-light radius $r_{e}$. Comparison of dispersions derived with various template star spectra (broadened and shifted to match the galaxy spectrum using the Fourier-space fitting code of van der Marel \& Franx 1993) indicates $\sim 5 \%$ systematic errors, which we convolve into the reported uncertainties. For ionized gas rotation velocities, we adopt a non-parametric statistical estimator of the maximum rotation velocity, the "probable min-max" $V_{\text {pmm }}$ of Raychaudhury et al. (1997) as implemented in KFF. The raw error for $V_{\mathrm{pmm}}$ is set to the $11 \mathrm{~km} \mathrm{~s}^{-1}$ systematic uncertainty estimated by KFF from comparison to radio linewidths (scaled from the $20 \mathrm{~km} \mathrm{~s}^{-1}$ scatter in the conversion $W_{50}=33+0.92\left(2 V_{\text {pmm }}\right)$ from KFF's Appendix B3). Stellar rotation velocities $V_{*}$ are also available for some galaxies, where we have applied the probable min-max technique to a rotation curve extracted with the IRAF cross-correlation task xcsao (Kurtz et al. 1992). Table 2 also provides the extent and asymmetry of the gas rotation curves (see Kannappan \& Barton 2004), as well as the slit P.A.s for both gas and stellar observations, with an asterisk in case the slit alignment with the galaxy major axis is uncertain or misaligned by more than $\sim 10^{\circ}$. Comparisons using (1) different instrumental setups and (2) different rotation curve extraction techniques indicate that both asymmetry and $V_{\text {pmm }}$ measurements are quite repeatable for rotation curves of reasonable $\mathrm{S} / \mathrm{N}$ (Barton et al. 2000; Kannappan \& Barton 2004), while extent measurements are somewhat more dependent on $\mathrm{S} / \mathrm{N}$.

Values of $V_{\text {pmm }}$ may be bracketed as unreliable in Table 2 if the ionized gas rotation curve is unlikely to adequately probe the potential well due to limited radial extent (e.g., Pisano et al. 2001; Kannappan \& Barton 2004). For morphological types earlier than Sc, a reliable rotation curve is defined to extend past $1.3 r_{e}$ (using the average extent of the two sides), which corresponds to the canonical turnover radius for large spiral galaxy rotation curves (KFF). For types Sc or later, we relax this criterion slightly in requiring only extent $>r_{e}$, because rotation curves that do not turn over are typical for very late-type galaxies and do not generally yield low- $V$ outliers in the Tully-Fisher relation. No values of stellar $\sigma$ are bracketed in Table 2 because data with inadequate $\mathrm{S} / \mathrm{N}$ or resolution to obtain reliable $\sigma$ values are not reported; likewise, we do not report $V_{*}$ when the stellar rotation curve extends to $<1.3 r_{e}$. Although all stellar kinematic measurements we report should therefore be reliable, dispersions marked with an $l$ should be used cautiously as dynamical mass estimators, due to the likelihood of substantial rotational support not accounted for in the stellar $\sigma$ alone (see the next section).

\subsubsection{Characteristic Velocity Assignments and Inclinations}

For each galaxy, we assign the largest reliable velocity derived from either gas or stellar kinematics as the characteristic internal velocity $V$ listed in Table 2 . In principle $V$ should be equal to the inclination-corrected gas rotation velocity for an idealized, purely dynamically cold system. Because in practice gas turbulence and other non-circular motions included in $\mathrm{H}$ I linewidths contribute to the dynamical support of galaxies, we adopt the inclination-corrected $\mathrm{H}_{\mathrm{I}}$ linewidth $W_{50} /(2 \sin i)$ as our fiducial gas-derived $V$, converting the ionized-gas $V_{\text {pmm }}$ to a pseudo- $W_{50}$ via the empirical optical-radio calibration in KFF. This approach restores non-rotational gas support to our ionizedgas-derived $V$ estimates in an average way (and is validated by the tighter Tully-Fisher relation obtained by Kassin et al. 2007 when they explicitly measure and include ionized gas dispersion for a high-redshift galaxy sample). To put stellar $V_{*}$ measurements on the same scale, we first multiply them by 1.1 (the typical $V_{\mathrm{pmm}} / V_{*}$ ratio measured in cases where both rotation curves extend to $>1.3 r_{e}$ ) and then scale them just like ionized gas $V_{\text {pmm }}$ measurements.

We further attempt to put rotation- and dispersion-derived estimates of $V$ on the same scale by applying the rule that for a pure dynamically hot system, $V$ equals $\sqrt{2} \times$ the stellar $\sigma$ (an approximation based on the scaling for an isothermal sphere; Burstein et al. 1997). However, low-mass E/S0 galaxies may be significantly supported by stellar rotation (Davies et al. 1983), and we cannot assess this support uniformly with the data in hand. In several cases, gas or stellar rotation curves confirm substantial rotational support, generally yielding strong outliers from the Faber-Jackson relation below $V \sim$ $125 \mathrm{~km} \mathrm{~s}^{-1}$ (Section 3). Thus, we consider stellar dispersionderived $V$ values unreliable for E-S0a galaxies with $V<$ $125 \mathrm{~km} \mathrm{~s}^{-1}$, except for two that are clearly dynamically hot, with dispersions consistent with the Faber-Jackson relation for highmass galaxies (NGC 3605 and NGC 4308). For the same reason we consider stellar dispersion-derived $V$ values unreliable for galaxies of type $\mathrm{Sa}$ or later. We mark low-mass/late-type $\sigma$ measurements with an $l$ in Table 2 to indicate likely unreliability. However, if such a $\sigma$ exceeds a reliable gas-derived $V$, then we accept the stellar-derived $V$ as reliable.

Superscripts $i r, n r, s r$, and $s d$ indicate the origin of the final $V$ estimates in Table 2 from ionized-gas rotation $V_{\text {pmm }}$ (79 galaxies), neutral gas rotation $W_{50}$ (53 galaxies), stellar rotation $V_{*}$ (12 galaxies), or stellar dispersion $\sigma_{r_{e} / 4}$ (47 galaxies), where 24, 17,2 , and 8 of the final $V$ values in each category are bracketed as unreliable, respectively, leaving 140 reliable final $V$ values. In combining the four types of kinematic data, we adopt two additional unreliability criteria besides those detailed in Sections 2.1.2 and 2.1.3: (1) although we assume that $\mathrm{H}_{\mathrm{I}} W_{50}$ measurements probe the full potential well for late-type galaxies, for types SOa and earlier we consider the $\mathrm{H}_{\mathrm{I}} W_{50}$ measurement unreliable if the ionized gas rotation curve is truncated, based on experience that $\mathrm{H}_{\mathrm{I}}$ in S0s often fails to probe the full potential well (KFF); and (2) for rotation-derived $V$ values, we treat the inclination correction as reliable only for $i>40^{\circ}$.

Our current best inclination angle estimates are provided in Table 2, mostly derived from photometric axial ratios as in KFF. A few values have been updated, with the old values used by KFF given in parentheses. For the polar ring galaxy UGC 9562 we adopt $i=68^{\circ}$ for the gas ring based on interferometric CO data (Wei et al. 2010b), and similarly for NGC 3499 we adopt $i=90^{\circ}$ for the gas (but not the stars) given the appearance of an edge-on dust lane in an otherwise round system. For NGC 7077 we list the $i=40^{\circ}$ inclination estimate from Wei et al. (2010b) for the record, although it just misses our inclination cut so the change does not affect our analysis. For UGC 6206 we adopt $i=43^{\circ}$ as a compromise between the highly discrepant 


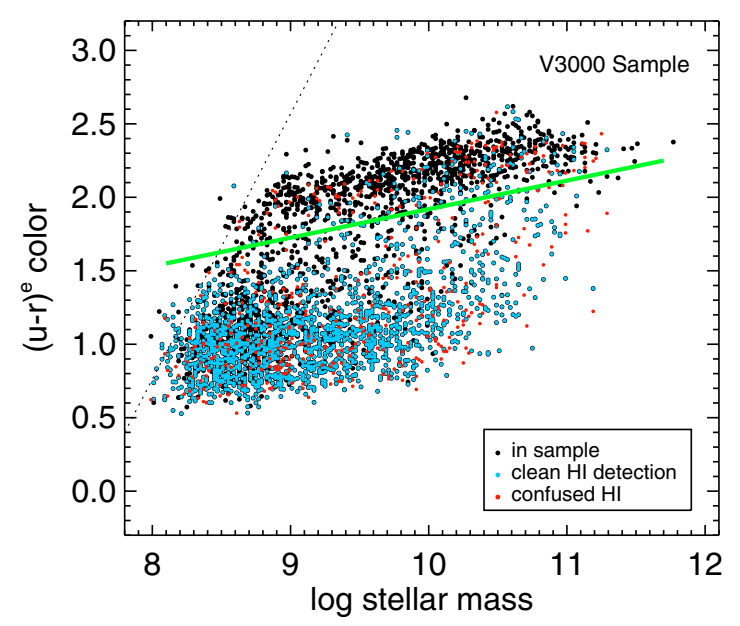

Figure 3. V3000 sample shown in $(u-r)^{e}$ color vs. stellar mass parameter space. As in Figure 1, we use de-extincted colors to enhance separation of the sequences. Galaxies with unconfused $\mathrm{H}$ I detections (blue dots) lie primarily on the blue sequence. Red dots mark possibly confused H I detections, which are excluded from the remainder of our analysis. The obviously slanting selection effect marked by the dashed line at the low-mass end of the plot reflects the fact that the $r$ band used to define the sample correlates most directly with baryonic rather than stellar mass, as shown in Figure 5 (see also Kannappan \& Wei 2008). (A color version of this figure is available in the online journal.)

axial ratios quoted by Mazzarella \& Boroson (1993) and Jansen et al. (2000b). For UGC $12265 \mathrm{~N}$, which has a very small angular size, the Jansen et al. axial ratio would be calculated with too few significant digits to give a meaningful constraint on the inclination, so we use the axial ratio from the NASA Extragalactic Database to estimate $i=57^{\circ}$. Finally, for the strongly distorted galaxy NGC 5993 we reduce the inclination to $i=32^{\circ}$ based on our analysis of the SDSS photometry (implying $i<40^{\circ}$ or "unreliable" status, consistent with this galaxy's extreme Tully-Fisher outlier behavior discussed in $\mathrm{KFF}$ ).

\subsection{The V3000 Sample}

The V3000 sample spans a redshift range of $2530-7000 \mathrm{~km} \mathrm{~s}^{-1}$ within two SDSS sky regions that offer uniform, public $\mathrm{H}$ I data from the blind $21 \mathrm{~cm}$ ALFALFA survey in the $\alpha .40$ data release (R.A. $130^{\circ}-237^{\circ} .5$, decl. $4^{\circ}-16^{\circ}$ and $24^{\circ}-28^{\circ}$; Haynes et al. 2011). Including all galaxies known to be in the volume down to $M_{r}=-17$, the sample comprises 3834 galaxies, not including 57 galaxies with irretrievable photometry (usually due to a missing/corrupted SDSS image or a very bright star). Of these,
1911 have clean Hi detections, 1527 have Hi upper limits, and 396 have possibly confused $\mathrm{H}$ I detections. The distribution of $\mathrm{HI}$ data in the color versus stellar mass diagram for the V3000 sample is shown in Figure 3. The high rate of upper limits $(\sim 40 \%)$ is obviously unsatisfying, but increasing the survey volume toward redshifts $<2500 \mathrm{~km} \mathrm{~s}^{-1}$ in order to add more detections would present substantial challenges for robust photometry and stellar mass estimation, while adding a modest number of galaxies and introducing a strong bias toward the Virgo Cluster. As defined, the V3000 sample reflects the natural diversity of galaxy environments ranging from voids to clusters (Figure 4).

The V3000 sample has been designed as a superset of a sample that would be complete to a limiting baryonic mass of $\sim 10^{9.3} M_{\odot}$, allowing for variable mass-to-light ratio. The $r$ band is optimal for selection on baryonic mass due to the modest scatter and luminosity dependence of $r$-band baryonic mass-to-light ratios (Figure 5; see also Kannappan \& Wei 2008). The nominal completeness limit of the SDSS redshift survey, Petrosian $r=17.77 \mathrm{mag}$ (using SDSS DR7 catalog magnitudes corrected for foreground extinction), corresponds to Petrosian $M_{r}=-17.23$ at $7000 \mathrm{~km} \mathrm{~s}^{-1}$, the far side of the V3000 volume. Our extrapolated $r$-band magnitudes are systematically brighter than catalog Petrosian magnitudes by $\sim 0.1$ dex (with large outliers; see Figure 5), due to improved sky subtraction and robust extrapolation (Section 2.1.1), so our equivalent limit is $M_{r} \sim-17.33$. We have furthermore reprocessed photometry for many galaxies near the survey limit, extending the sample down to $M_{r}=-17$ where redshifts are available and recovering bright galaxies shredded by the SDSS pipeline. For this effort, we have made use of a merged redshift catalog that draws on SDSS DR6/DR7/DR8, Updated Zwicky Catalog, HyperLEDA, ALFALFA, 6dF, 2dF, and GAMA data (Adelman-McCarthy et al. 2008; Abazajian et al. 2009; Aihara et al. 2011; Falco et al. 1999; Paturel et al. 2003; Haynes et al. 2011; Martin et al. 2010; Jones et al. 2009; Colless et al. 2003; Driver et al. 2011). Roughly $8 \%$ of the V3000 sample above $M_{r}=-17.33$ has been recovered in this way. It is worth noting that most of the extra redshifts come from historical surveys with fairly bright limiting magnitudes, so our inventory of low surface brightness dwarfs remains incomplete.

The scatter in Figure 5(a) suggests high completeness to $M_{r}=$ -17.73 , corresponding to a typical baryonic mass of $\sim 10^{9.1} M_{\odot}$, as shown in Figure 5(b). However, variations in baryonic massto-light ratio up to $\sim 3$ would mandate extension to $M_{r} \sim-17$ to retain unbiased completeness at $M_{\text {bary }} \sim 10^{9.1} M_{\odot}$. As we have only partial completeness from $M_{r}=-17$ to -17.33 , we

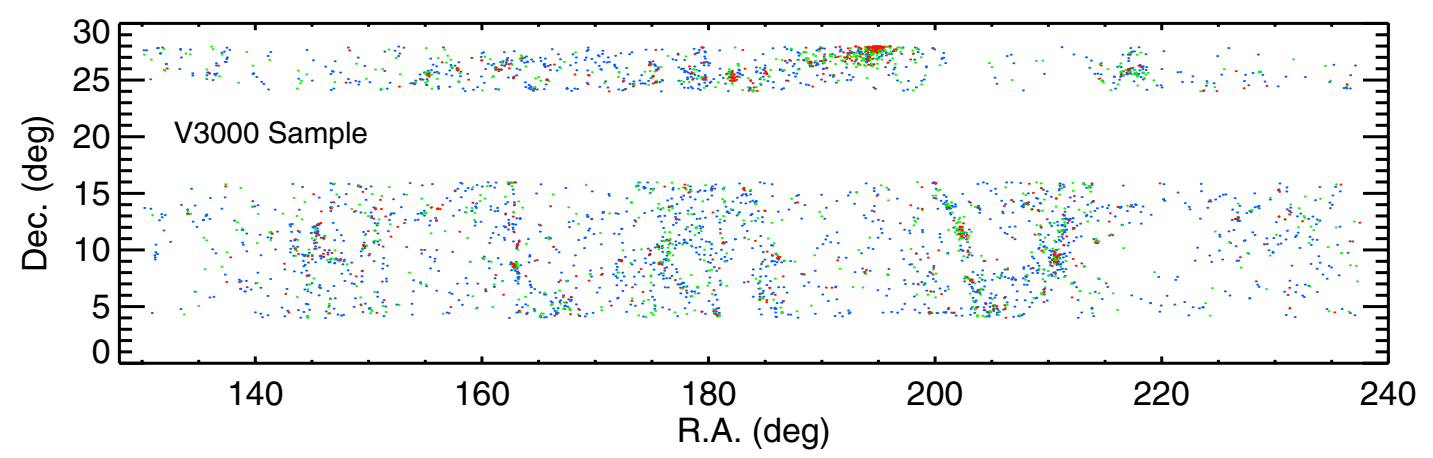

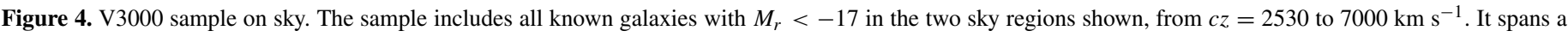

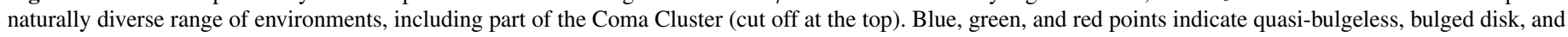
spheroid-dominated galaxies based on $\mu_{\Delta}$ class (Figure 1). Points are overplotted in that order to highlight sites of spheroid-dominated galaxy concentration.

(A color version of this figure is available in the online journal.) 

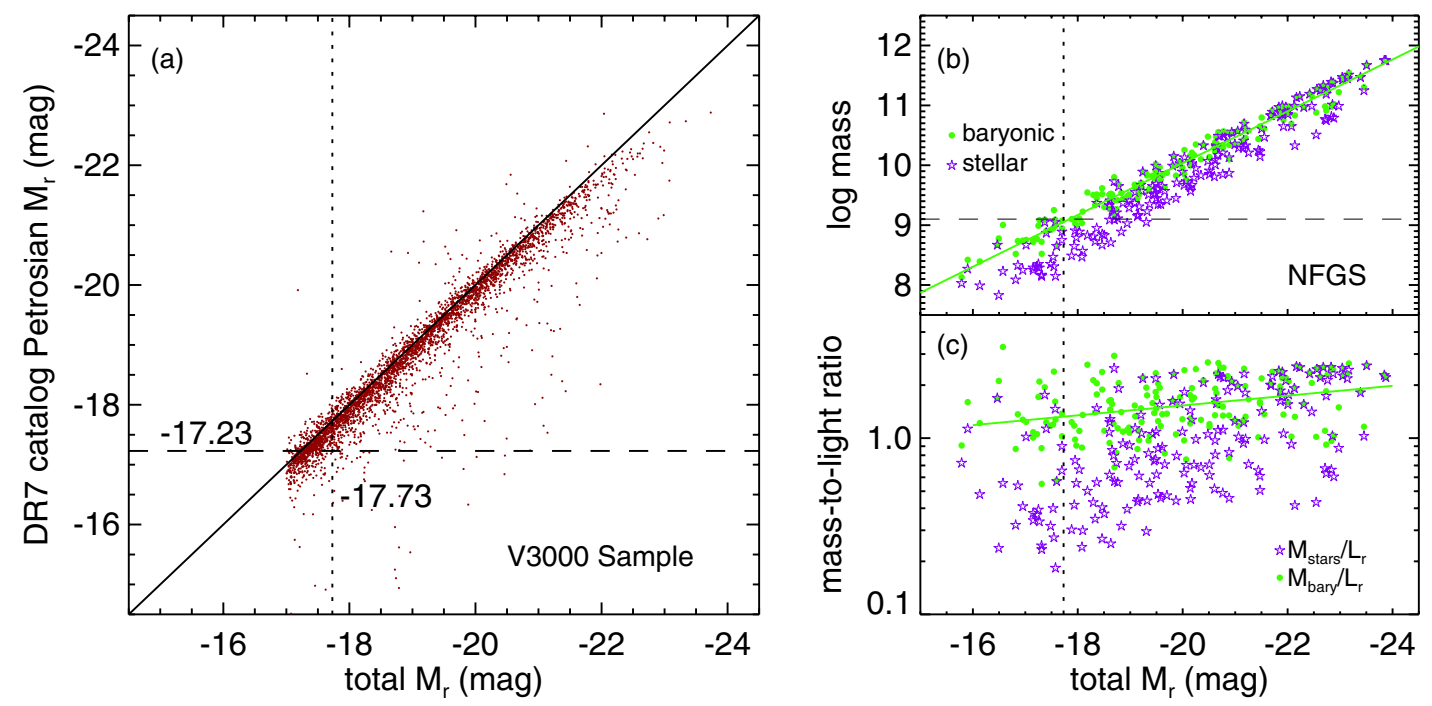

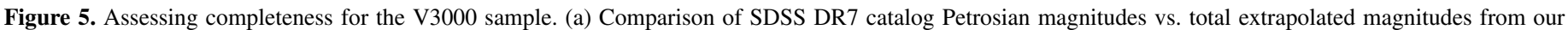

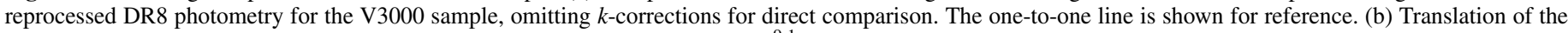

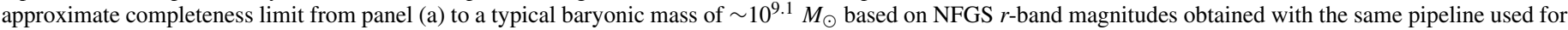

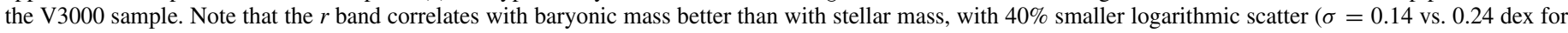

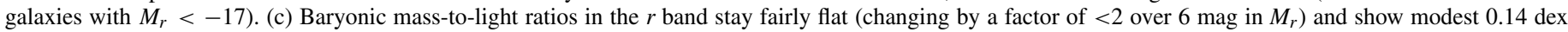

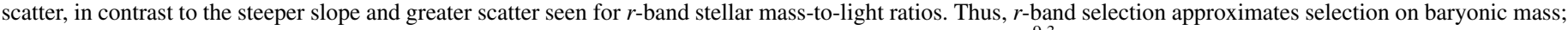
nonetheless, scatter up to mass-to-light ratios of $\sim 3$ implies a final baryonic mass completeness limit of $\sim 10^{9.3} M_{\odot}$.

(A color version of this figure is available in the online journal.)

estimate that the $\mathrm{V} 3000$ sample is complete to $M_{\text {bary }} \sim 10^{9.3} M_{\odot}$ and $M_{*} \sim 10^{9.1} M_{\odot}$. We define an initial approximately baryonic mass limited sample by $M_{r}<-17.73$, which yields 3020 galaxies, of which 1510 have unconfused Hi detections and 1171 have upper limits. In Section 4.4.1 we attempt to define a more precisely baryonic mass-limited sample by estimating gas content via "photometric gas fractions" (K04) for galaxies with upper limits and then selecting all galaxies with $M_{\text {gas }}+M_{*}>$ $10^{9.3} M_{\odot}$ down to $M_{r}=-17$.

Photometric measurements for the V3000 sample have been performed as part of the ongoing construction of two other volume-limited surveys not restricted to the ALFALFA $\alpha .40$ footprint (RESOLVE, REsolved Spectroscopy Of a Local VolumE; S. J. Kannappan et al., in preparation; and a larger survey encompassing both the RESOLVE and V3000 samples; A. J. Moffett et al., in preparation). These surveys will enable analysis within environmental context, which we defer to future work. We reprocess GALEX, SDSS DR8, and 2MASS imaging in the same way as for the NFGS (Section 2.1.1); GALEX imaging is available for $\sim 30 \%$ of the V3000 sample. Stellar-mass estimates for the V3000 sample are derived from the NUV+ugrizJHK magnitudes using the same updated model grid used to determine new mass estimates for the NFGS (Section 2.1.1). From a comparison of colors and masses for the V3000 sample and the SDSS-derived "HyperLEDA+" sample of KGB, we conclude that our new photometry and updated model grid together yield similar, slightly lower stellar masses and $\sim 0.2$ mag bluer $u-r$ colors than would be obtained with SDSS catalog photometry, consistent with the discussion in Section 2.1.1.

For V3000 galaxies with $21 \mathrm{~cm}$ detections we obtain H I fluxes and $W_{50}$ linewidths from ALFALFA catalog measurements, implying likely underestimation of the true characteristic velocity in the case of gas-poor systems. We therefore treat $W_{50}$ as unreliable for detections below $M_{\mathrm{HI}} / M_{*}=0.1$, which rejects a large fraction of the outliers in the ALFALFA $W_{50}$-based Tully-Fisher relation. Formal errors from the ALFALFA catalog typically imply $\lesssim 5-10 \mathrm{~km} \mathrm{~s}^{-1}$ error on $W_{50} /(2 \sin i)$, so we treat cases with $>20 \mathrm{~km} \mathrm{~s}^{-1}$ error as unreliable as well. In either case, when our analysis requires a velocity we use the equivalent $V$ inferred from the $r$-band absolute magnitude-velocity relation $\log V=$ $-0.29-0.123 M_{r}$ (calibrated using all galaxies with reliable $V$ and $M_{r}<-17$ in the NFGS; this calibration is very slightly different from that in Figure 2 due to evolution in the photometry after the $\mathrm{H}$ I measurements were performed). An $M_{r}$-inferred $V$ is also used to compute $M_{\mathrm{HI}}$ upper limits (defined at $5 \sigma$ ); we combine the inferred $V$ with the measured inclination angle and the declination-dependent ALFALFA rms noise to derive the limit value as well as provide a $W_{50} /(2 \sin i)$ surrogate. Finally, because we estimate inclinations for the V3000 sample from axial ratios determined by our automated pipeline, we cannot individually vet inclination estimates in the borderline $i=40^{\circ}-50^{\circ}$ range as we could for the NFGS, so $W_{50} /(2 \sin i)$ is designated unreliable for $i<50^{\circ}$ and again replaced with an $M_{r}$-inferred $V$.

Possible confusion of $\mathrm{H}$ I sources is flagged within a $3^{\prime}$ radius, which combines the $2^{\prime}$ half-power radius of the smoothed ALFALFA resolution element with another $1^{\prime}$ to allow for the typical $\sim 0.5-1^{\prime}$ diameters of galaxies in this redshift range. We assume catalog redshift uncertainties (minimum $50 \mathrm{~km} \mathrm{~s}^{-1}$ ) and $\sim 100 \mathrm{~km} \mathrm{~s}^{-1}$ profile widths (the approximate average of the $\mathrm{H} \mathrm{I}$ velocity width function of Papastergis et al. 2011) to assess potential overlap, employing the same merged redshift catalog described above. Potentially confused detections are excluded from our $\mathrm{H}$ I analysis but are shown as red dots in Figure 3.

\section{DISTINGUISHING THE GAS-RICHNESS THRESHOLD AND BIMODALITY SCALES}

Figure 6 combines the Tully-Fisher and Faber-Jackson relations for the NFGS into a unified mass-velocity $(M-V)$ relation for stellar mass in panel (a) and baryonic mass in panel (b), illustrating multiple equivalent definitions of the threshold and bimodality scales in terms of $V, M_{*}$, and $M_{\text {bary }}$ (stars + cold 

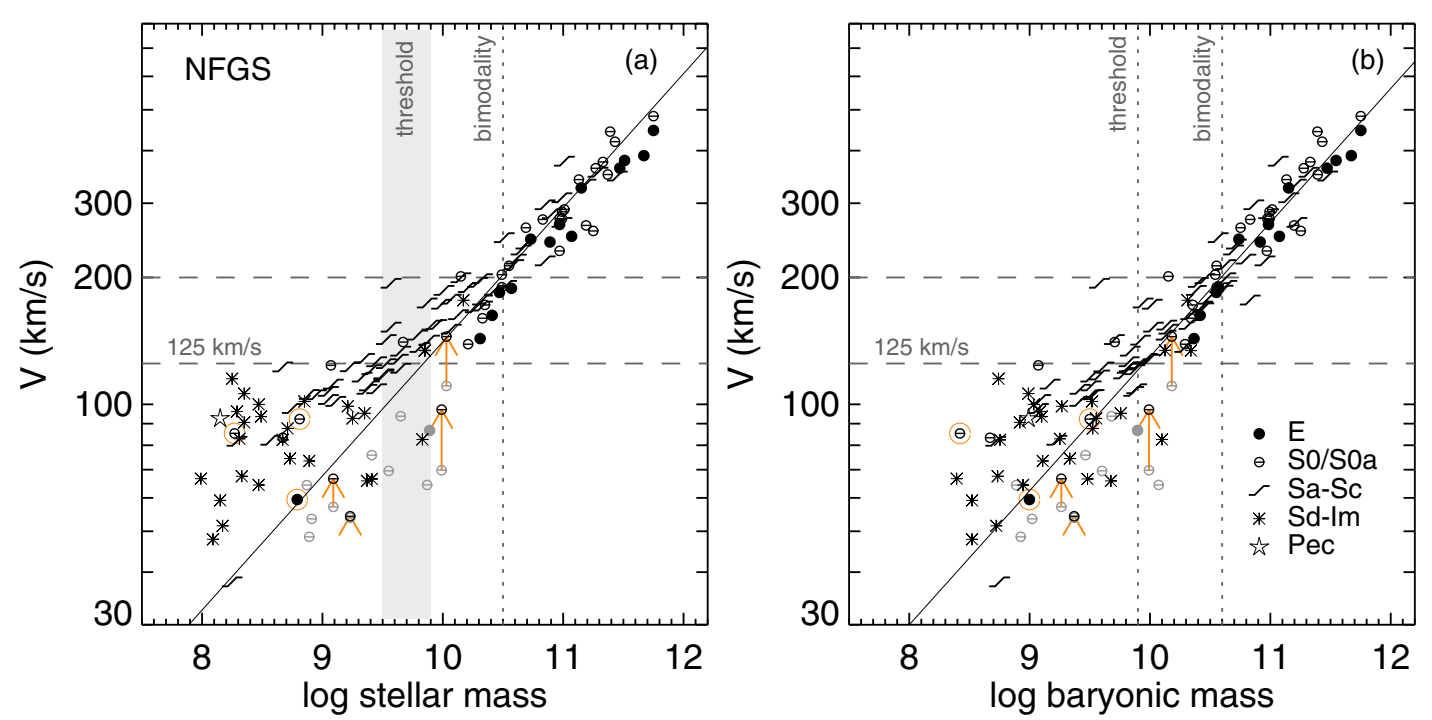

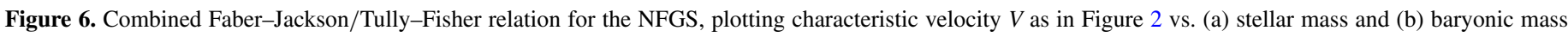

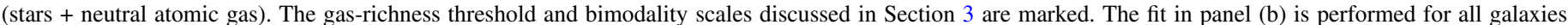

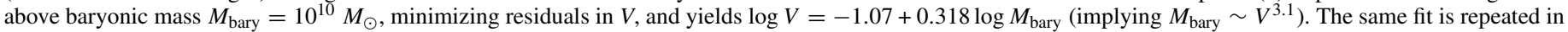

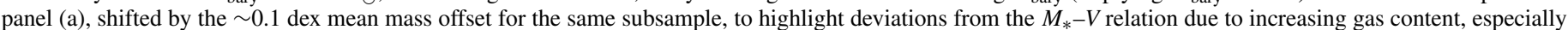

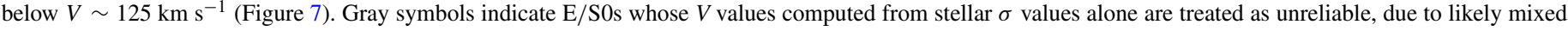

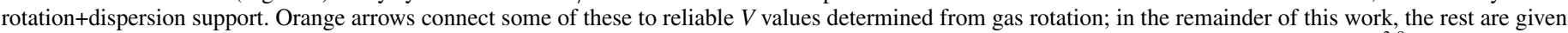

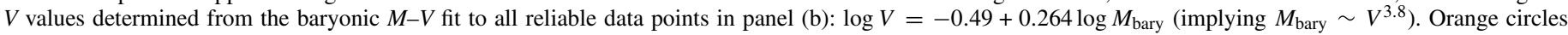
indicate additional $\mathrm{E} / \mathrm{S} 0 \mathrm{~s}$ with gas-derived $V$ values, in this case with no stellar $\sigma$ values for comparison.

(A color version of this figure is available in the online journal.)

gas). The characteristic velocity $V$ used is the optimal choice of stellar or gas-derived internal velocity for each galaxy as described in Section 2.1.4. The bimodality scale corresponds to the $M_{*} \sim 10^{10.5} M_{\odot}$ scale highlighted by Kauffmann et al. (2003a), which we identify with $V \sim 200 \mathrm{~km} \mathrm{~s}^{-1}$ in Figure 6. The threshold scale corresponds to the $V \sim 125 \mathrm{~km} \mathrm{~s}^{-1}$ scale highlighted by Garnett (2002) and Dalcanton et al. (2004) ${ }^{19}$, which we identify with $M_{\text {bary }} \sim 10^{9.9} M_{\odot}$ in Figure 6. The wide range of gas-to-stellar mass ratios at the threshold scale (Figure 7) permits no well-defined stellar mass equivalent, but a reasonable fiducial is $M_{*} \sim 10^{9.7} M_{\odot}$ in the middle of the 0.4 dex band shown.

The gas-richness threshold and bimodality scales are distinguished by simultaneous changes in structure (morphology/ dynamics) and gas-richness. Figure 1(a) shows that quasibulgeless Sd-Im morphologies ("dwarf" late types) become notably more common below the threshold scale, while spheroiddominated E-SOa types become similarly abundant above the bimodality scale. This pattern is consistent with previous work showing the emergence of bulges above the threshold scale (Dalcanton et al. 2004; Bell 2008) and the transition from diskdominated to spheroid-dominated systems above the bimodality scale (Kauffmann et al. 2003a). In the NFGS, traditional Sa-Sc spirals are most prominent in the narrow mass range between the threshold and bimodality scales, but whether they numerically predominate is unclear. We defer consideration of this question to Section 4.4.3, where we will use the volume-limited V3000 sample to examine what defines the transition range between the threshold and bimodality scales.

Symbol colors in Figure 1(a) indicate quasi-bulgeless, bulged disk, and spheroid-dominated galaxies according to our $\mu_{\Delta}$ metric (Section 2.1.1 and Figure 1(b)), which combines overall

19 Dalcanton et al. adopt a slightly different $V=120 \mathrm{~km} \mathrm{~s}^{-1}$ value as compared to Garnett's $125 \mathrm{~km} \mathrm{~s}^{-1}$ value adopted here. surface mass density with central versus outer-disk surface mass density contrast. We see that below the threshold scale, most NFGS galaxies typed as spirals are quasi-bulgeless, while earlier type galaxies, particularly those on the blue sequence, often have $\mu_{\Delta}$ comparable to that of $\mathrm{Sa}-\mathrm{Sc}$ spirals. The latter result is consistent with the idea that low-mass blue-sequence E/S0s are associated with disk rebuilding after gas-rich mergers, likely leading to the regeneration of late-type morphologies (KGB; W10a; Wei et al. 2010b; Moffett et al. 2012; S13). Like spirals, S0s display the full range of $\mu_{\Delta}$ classes (as also emphasized by the classification system of van den Bergh 1976).

E/S0s above the bimodality scale are generally dynamically hot (as judged by the fact that stellar velocity dispersions alone can provide most of the virial support required for the $M-V$ relation ${ }^{20}$; Figure 6), but the E/S0 population diversifies at lower masses, with only a minority remaining dynamically hot. For $\mathrm{E} / \mathrm{S} 0 \mathrm{~s}$ just below the bimodality scale, the scatter in dispersionderived $V$ estimates stays within $\sim 20 \%$ of the baryonic and stellar $M-V$ relations, but below the threshold scale, severe $M-V$ outliers would be common if we accepted stellar $\sigma$ values as reliable estimators of $V$ (light gray symbols in Figure 6(a)). Significant rotational support most naturally explains this outlier behavior, as confirmed for a few cases that have reliable gas-derived $V$ values (see $V$ estimates connected with orange arrows in Figure 6(b)). In keeping with this interpretation, the low-mass E/S0s whose only reliable $V$ is gas derived are not

\footnotetext{
20 It is interesting that massive S0 and early-type spiral galaxies may have photometrically prominent disks and yet still have sufficiently high stellar $\sigma$ values to account for most of their virial support. Barway et al. (2007, 2009) find that lenticular galaxies above a $K$-band magnitude of -24.5 (which roughly equates to $M_{*} \sim 10^{10.8-10.9} M_{\odot} ; \mathrm{KGB}$ ) have bulges consistent with formation involving mergers rather than pure secular evolution. The final analysis of Laurikainen et al. (2010, updating their earlier papers) also confirms that lenticular galaxies above this luminosity behave more like ellipticals in the central surface brightness versus effective radius relation than like pseudobulge-dominated SOs, which are more typical at lower luminosities.
} 

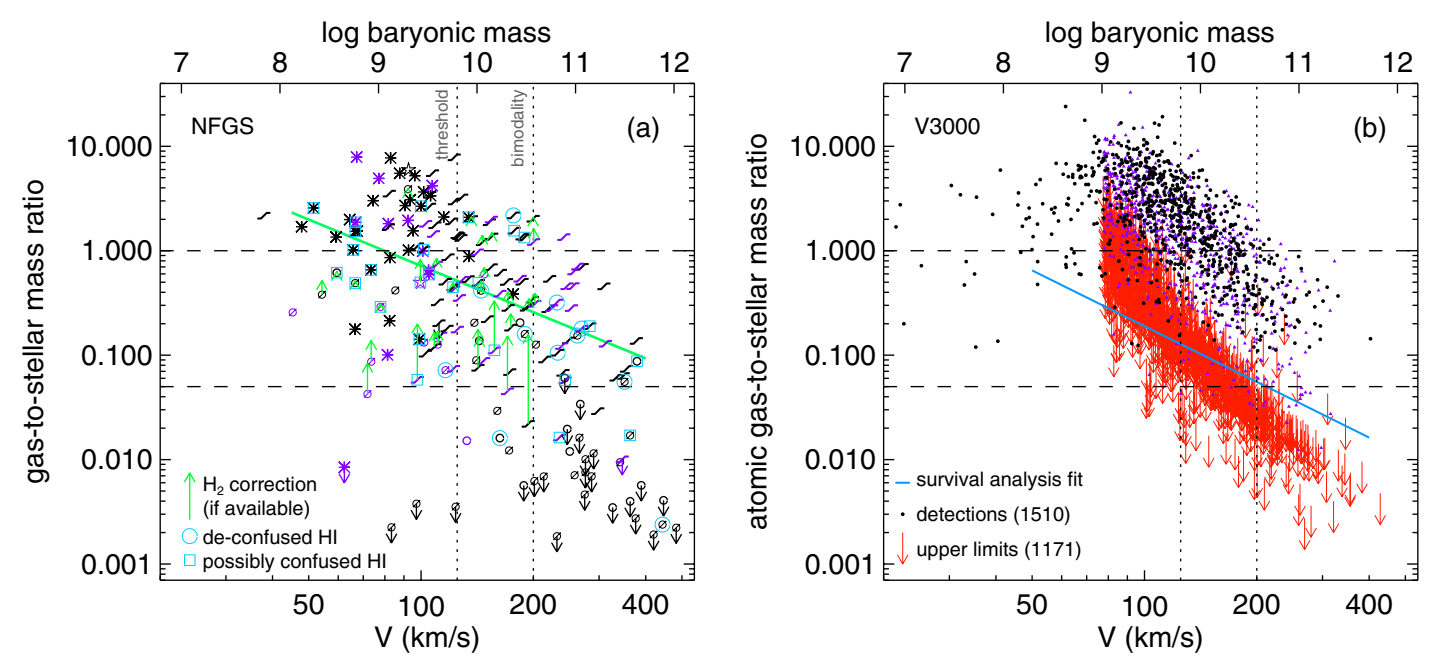

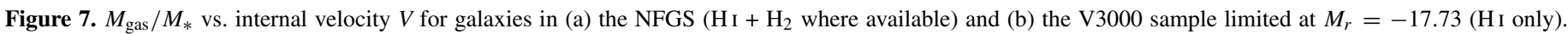

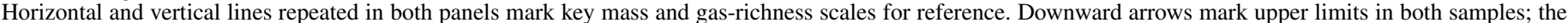

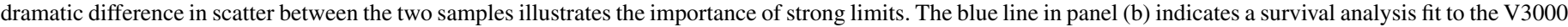

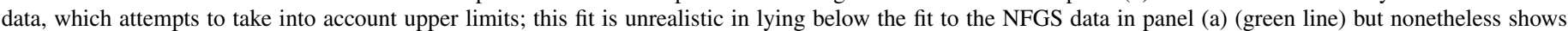

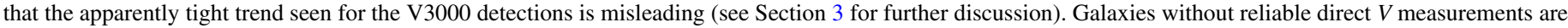

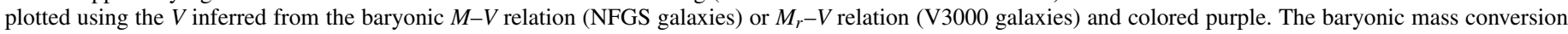

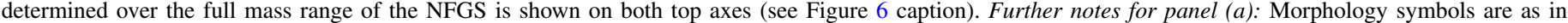

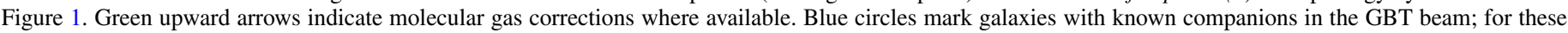

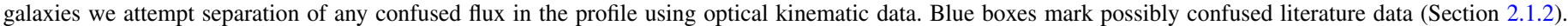

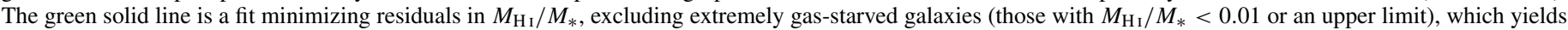

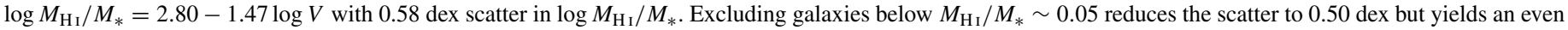
shallower slope: $\log M_{\mathrm{H}_{\mathrm{I}}} / M_{*}=2.40-1.24 \log V$.

(A color version of this figure is available in the online journal.)

outliers (circled in orange in Figure 6). We infer that most $\mathrm{E} / \mathrm{S} 0$ s are rotation supported below the threshold scale (and therefore treat their $V$ measurements as unreliable unless they are unambiguously dynamically hot or have reliable gas-derived velocities). This population includes many blue-sequence $\mathrm{E} / \mathrm{S} 0$ s in low-density environments, consistent with a formation scenario involving increasingly gas-dominated mergers below the threshold scale, as expected from Figure 7 (and logically extending the wet/dry merger scenario used by Emsellem et al. 2007 to explain the fast/slow rotator dichotomy for E/S0s above the bimodality scale).

Late-type galaxies also deviate from the stellar $M-V$ relation below the threshold scale, in the sense of higher $V$ at a given mass. However, this deviation is diminished by the inclusion of $M_{\mathrm{H} \text { I }}$ to recover a tight "baryonic" Tully-Fisher relation (McGaugh et al. 2000). The onset of significant deviations is most easily seen by comparing the stellar $M-V$ relation to the fit from the baryonic $M-V$ relation, which is derived for all galaxies with $M_{\text {bary }}>10^{10} M_{\odot}$ in Figure 6(b) and shifted to the mean $M_{*}$ for the late types among these galaxies in Figure 6(a). The gas mass correction becomes important below $V \sim 125 \mathrm{~km} \mathrm{~s}^{-1}$, exactly where gas-dominated galaxies become abundant (Figure 7). It is intriguing that even after we add $M_{\mathrm{HI}}$, the $M-V$ relation remains slightly offset to higher $V$ below $V \sim 125 \mathrm{~km} \mathrm{~s}^{-1}$. The remaining offset could reflect either a change in relative baryonic-to-dark matter concentration below the threshold scale or an additional undetected gas reservoir. Previous studies of the baryonic Tully-Fisher relation have argued that adding a multiple of the $\mathrm{H}$ I-derived gas mass yields the tightest $M-V$ relation, implying the possibility of undetected gas in ionized, CO-dark molecular, or optically thick atomic form (Pfenniger \& Revaz 2005; Begum et al. 2008).

The threshold and bimodality scales are associated with two physically significant transitions in galaxy gas content, seen most clearly in the NFGS (Figure 7(a)): below the threshold scale, gas-dominated galaxies with gas-to-stellar mass ratios $M_{\mathrm{HI}} / M_{*}>1$ become noticeably more abundant, while above the bimodality scale, gas-starved galaxies with $M_{\mathrm{HI}} / M_{*}<0.05$ start to predominate. ${ }^{21}$ Molecular gas corrections are provided where $\mathrm{CO}$ data are available (green arrows) and do not significantly affect the trends; $\mathrm{H}_{2}$ contributions generally become significant only when overall gas content is low. To a significant extent, bulgeless and bulge-dominated galaxies correspond to gas-dominated and gas-starved galaxies, respectively. However, S0-S0a galaxies are not always bulge-dominated (see Figure 1(b)), and they may have significant gas (as reported by many authors; e.g., Hawarden et al. 1981; Sage \& Welch 2006), which complicates analysis by Hubble type.

Patterns of gas content are obscured by weak upper limits in the V3000 sample (Figure 7(b)), creating a misleadingly tight trend. To illustrate the extent of the problem, the blue line in Figure 7(b) shows a fit using survival analysis to include upper limits. Specifically, we make use of the Buckley-James estimator (discussed as most robust to non-Gaussian scatter by Isobe et al. 1986), as implemented in the ASURV package (Rev. 1.2; Lavalley et al. 1992). The survival analysis fit lies well below the detected data and in fact even below the conventional fit for the NFGS data in Figure 7(a). However, we stress that the survival analysis result should not be overinterpreted, because survival analysis assumes that the incidence of upper limits is uncorrelated with the underlying values, an assumption often treated as roughly valid for flux-limited surveys but clearly

\footnotetext{
21 The choice of $M_{\mathrm{HI}} / M_{*} \lesssim 0.05$ as a transitional value is motivated by the fact that nearly all spiral-type systems are detected with $M_{\mathrm{HI}} / M_{*}>0.05$; however, the preponderance of upper limits for E/S0s even down to $M_{\mathrm{HI}} / M_{*} \lesssim 0.002$ leaves open the possibility that typical $M_{\mathrm{HI}} / M_{*}$ values for gas-starved E/S0s may actually be much lower than 0.05 .
} 
violated once distance limits are imposed as in the volumelimited V3000 sample. In Section 4.4.1 we will use an updated calibration of the photometric gas fraction technique (K04) to show that the full V3000 $M_{\mathrm{HI}} / M_{*}$ distribution likely looks quite similar to that of the NFGS.

In fact, the lack of a tight relationship between $M_{\mathrm{H}_{\mathrm{I}}} / M_{*}$ and $V$ for the NFGS may be the most important result seen in Figure 7. We stress that the extreme diversity of $M_{\mathrm{HI}} / M_{*}$ in the NFGS is real, reflecting its selection as a broadly representative sample of the galaxy population, whereas the seemingly tighter $M_{\mathrm{HI}} / M_{*}$ versus $V$ relation for the V3000 sample is created by weak upper limits as just discussed. Moreover, other data sets that may have seemed to show a tight dependence of gas content on galaxy mass have also been inherently detection biased and/or selection biased (for example, as in the work of K04 and McGaugh 2005, both widely used to estimate gas mass from stellar mass, e.g., as in Stewart 2011). We also note that past studies have generally plotted $M_{\mathrm{H}} / M_{*}$ versus $M_{*}$, in which case the usual $\gtrsim 0.2$ dex errors in $M_{*}$ combined with the covariance between plot axes will spuriously enhance the impression of a correlation. Figure 7 shows gas-richness transitions using $V$ as our preferred mass proxy, since it correlates tightly with baryonic mass and avoids covariance with $M_{\mathrm{HI}} / M_{*}$. With this choice, we do see some measurable trend in $M_{\mathrm{HI}} / M_{*}$ versus $V$, but the large scatter around the fit ( $\gtrsim 0.5$ dex excluding quenched galaxies) implies little predictive power not already contained in the statement that gas-dominated galaxies start to appear in large numbers below the threshold scale. Furthermore, compared to gas-dominated galaxies, gas-starved galaxies appear less closely tied to their characteristic mass scale, with examples at all galaxy masses in spite of the potential bias against gas-poor dwarfs in the $B$-selected NFGS.

Previous work has suggested abrupt changes in ISM physics at the threshold scale (Dalcanton et al. 2004), so the question of whether gas-richness is changing sharply at $V \sim 125 \mathrm{~km} \mathrm{~s}^{-1}$ in Figure 7 is of interest. The small number statistics of the NFGS do not allow us to establish whether the transition is sharp or continuous in that sample. The V3000 sample is larger, but also fails to confirm an abrupt transition, although a sharp transition could perhaps be hidden by its larger measurement uncertainties. Regardless, we will argue in Section 4 that galaxy mass (or its proxy $V$ ) is not the most fundamental variable underlying transitions in gas richness, and that the broad scatter in $M_{\mathrm{HI}} / M_{*}$ at low $V$ implies additional physics.

\section{GAS RICHNESS, LONG-TERM FSMGR, AND REFUELING REGIMES}

We have argued that distinct transitions occur at the threshold and bimodality scales, coupling gas-richness changes with structural changes. Yet we have also seen considerable diversity in galaxy properties at any given mass. Here we demonstrate that U-NIR colors perform far better than galaxy masses in predicting gas-richness and morphology. We show that these colors can be understood as proxies for the long-term fractional stellar mass growth rate, FSMGR $\mathrm{LT}_{\mathrm{LT}}$, averaged over the last Gyr. We further argue that the most natural interpretation of the tight $M_{\mathrm{HI}} / M_{*}$ versus FSMGR $\mathrm{LT}_{\mathrm{LT}}$ relation involves routine fresh gas accretion, and we identify three distinct regimes in FSMGR $\mathrm{FT}_{\mathrm{LT}}$, $M_{\mathrm{HI}} / M_{*}$, and morphology that we link to qualitatively different states of external gas accretion and internal gas processing ("refueling regimes"). Finally, we examine the mass dependence of the refueling regimes to show how they give rise to the threshold and bimodality scales.

\subsection{The Tight Relation of $M_{\mathrm{HI}} / M_{*}$ versus $U-N I R$ Color}

U-NIR colors define a surprisingly tight correlation with $M_{\mathrm{HI}} / M_{*}$, providing the basis for the photometric gas fraction technique of K04. In contrast to the $\sigma \sim 0.58$ dex scatter in the $M_{\mathrm{HI}} / M_{*}$ versus $V$ relation (Figure $7(\mathrm{a})$ ), we measure 0.36 dex scatter in the $M_{\mathrm{HI}} / M_{*}$ versus $(u-J)^{m}$ color relation (Figure 8(a)), using the same subset of NFGS galaxies (i.e., excluding extremely gas-starved systems with $M_{\mathrm{H}} / M_{*}<0.01$ or upper limits). We obtain 0.30 dex scatter in $M_{\mathrm{HI}} / M_{*}$ versus $(u-J)^{m}$ when excluding gas-starved systems with $M_{\mathrm{HI}} / M_{*}<$ 0.05 , as compared to the 0.50 dex scatter measured for the same galaxies in $M_{\mathrm{HI}} / M_{*}$ versus $V$. Moreover, since exclusions based on gas content are impractical from the point of view of predicting gas content, it is especially interesting that gasstarved galaxies are tightly confined in Figure 8(a) to colors redder than $(u-J)^{m}=3.7$. U-NIR color alone does not separate these gas-starved, spheroid-dominated galaxies from the reddest bulged disks (which have non-negligible gas and continue the $M_{\mathrm{HI}} / M_{*}$ versus $(u-J)^{m}$ correlation). Galaxies bluer than $(u-J)^{m}=3.7$ display 0.34 dex scatter around the fit shown, excluding the one outlier, whose nature is uncertain. ${ }^{22}$ As the typical stellar mass uncertainty for the NFGS is $0.15 \mathrm{dex}$, and systematic errors in stellar mass estimation are likely to be significantly larger (see Kannappan \& Gawiser 2007), measurement uncertainties likely contribute much of the observed scatter in Figure 8(a), implying an impressively tight underlying correlation.

The $M_{\mathrm{HI}} / M_{*}$ versus $(u-J)^{m}$ relation given in Figure 8(a) supersedes that of K04, with higher quality data (reprocessed SDSS and 2MASS imaging; carefully vetted, largely new H I measurements), superior stellar mass estimation (from SED modeling rather than the $g-r$ versus $M_{*} / L_{K}$ calibration of Bell et al. 2003), and a more representative galaxy sample spanning the natural diversity of galaxy gas-richness (as opposed to the inhomogeneous literature compilation used by K04). Given the far greater intrinsic diversity of the NFGS compared to prior samples used to calibrate the photometric gas fraction technique (K04; Zhang et al. 2009; Catinella et al. 2010; Li et al. 2012), it is remarkable that the scatter in our U-NIR relation is the same as has been obtained by these authors only by combining multiple parameters (e.g., optical color, surface brightness, stellar mass, and/or color gradient). We infer that U-NIR colors capture the physics of galaxy gas richness in an essential way, to be discussed in Section 4.2. However, we also note that internal extinction is responsible for some fortuitous straightening and tightening of the $(u-J)^{m}$ relation: if replotted using the extinction-corrected $(u-J)^{e}$, Figure 8 appears more similar to the bowed NUV $-r$ relation (e.g., as reported by Catinella et al. 2010). Moreover, our reprocessed $u-r$ colors (unlike SDSS catalog $u-r$ colors) are comparable to U-NIR colors in predictive power (K. D. Eckert et al., in preparation).

\subsection{U-NIR Color as a Long-term FSMGR Metric}

As shown in Figure 8(b), the primary physics underlying the $(u-J)^{m}-M_{\mathrm{HI}} / M_{*}$ correlation is another tight correlation,

\footnotetext{
22 The plot location of the outlier in Figure 8(a), UGC 4879, is either spurious or intriguing, as it is both (1) a post-starburst galaxy, with strong Balmer absorption and no detectable $\mathrm{H} \alpha$ emission; and (2) very nearby, with individually resolved star clusters and clumpy, irregular structure, making our photometry highly unreliable (Section 2.1.1).
} 

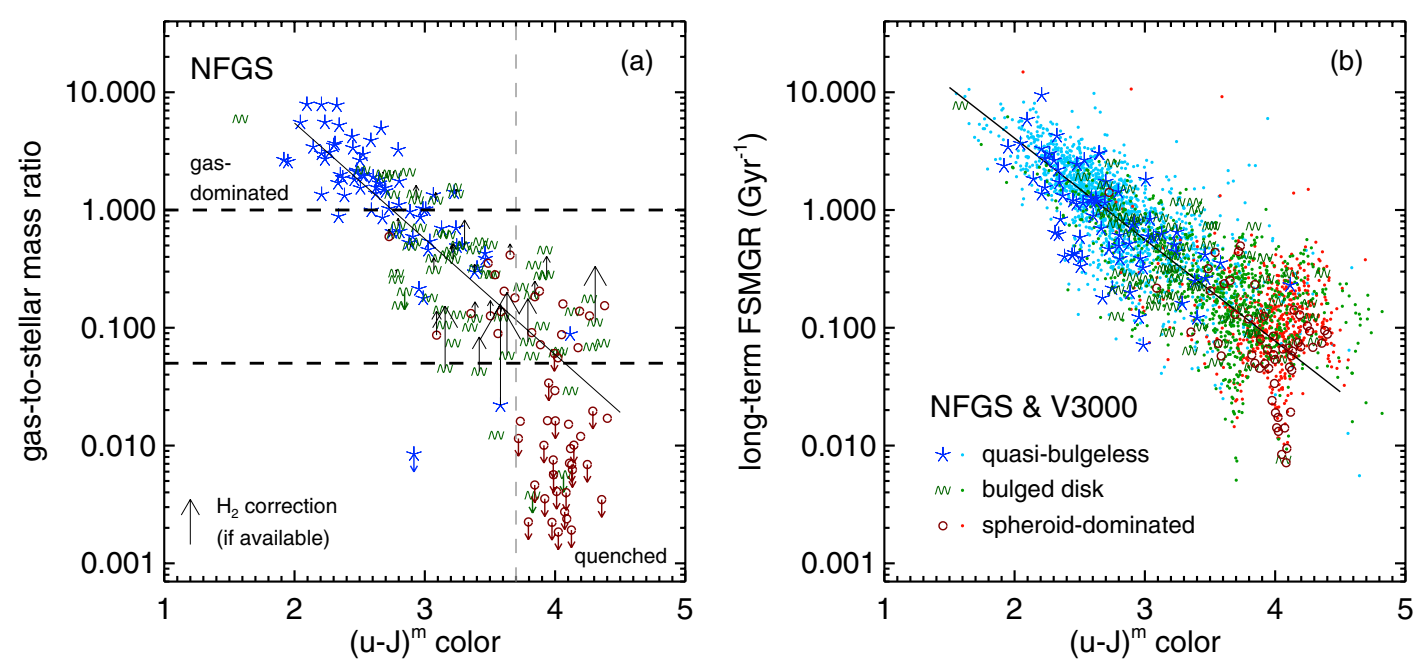

Figure 8. Gas-richness and long-term fractional stellar mass growth rate FSMGR $\mathrm{LT}_{\mathrm{L}}$ in relation to U-NIR color, with symbols indicating $\mu_{\Delta}$ morphology class as defined in Figure 1(b). (a) $M_{\mathrm{HI}} / M_{*}$ vs. $(u-J)^{m}$ color for the NFGS. The solid line is a fit minimizing residuals in $M_{\mathrm{H}_{\mathrm{I}}} / M_{*}$ for all galaxies bluer than $(u-J)^{m}=3.7$ (excluding the outlier, see footnote 22), which yields $\log M_{\mathrm{HI}} / M_{*}=2.70-0.98(u-J)^{m}$ with 0.34 dex scatter. If available, molecular gas corrections are shown with black arrows but are not used in the fit. (b) Mapping of $(u-J)^{m}$ color to FSMGR $\mathrm{LT}_{\mathrm{LT}}$, determined by fitting to a suite of two-component old+young stellar population models as described in Section 4.2. Small dots show V3000 sample galaxies with $M_{r}<-17.73$. We measure 0.30 dex rms scatter around the V3000 sample fit (black line: $\log \mathrm{FSMGR}_{\mathrm{LT}}=2.33-0.861(u-J)^{m}$ for galaxies bluer than $\left.(u-J)^{m}=3.7\right)$, which is comparable to the scatter in panel (a).

(A color version of this figure is available in the online journal.)
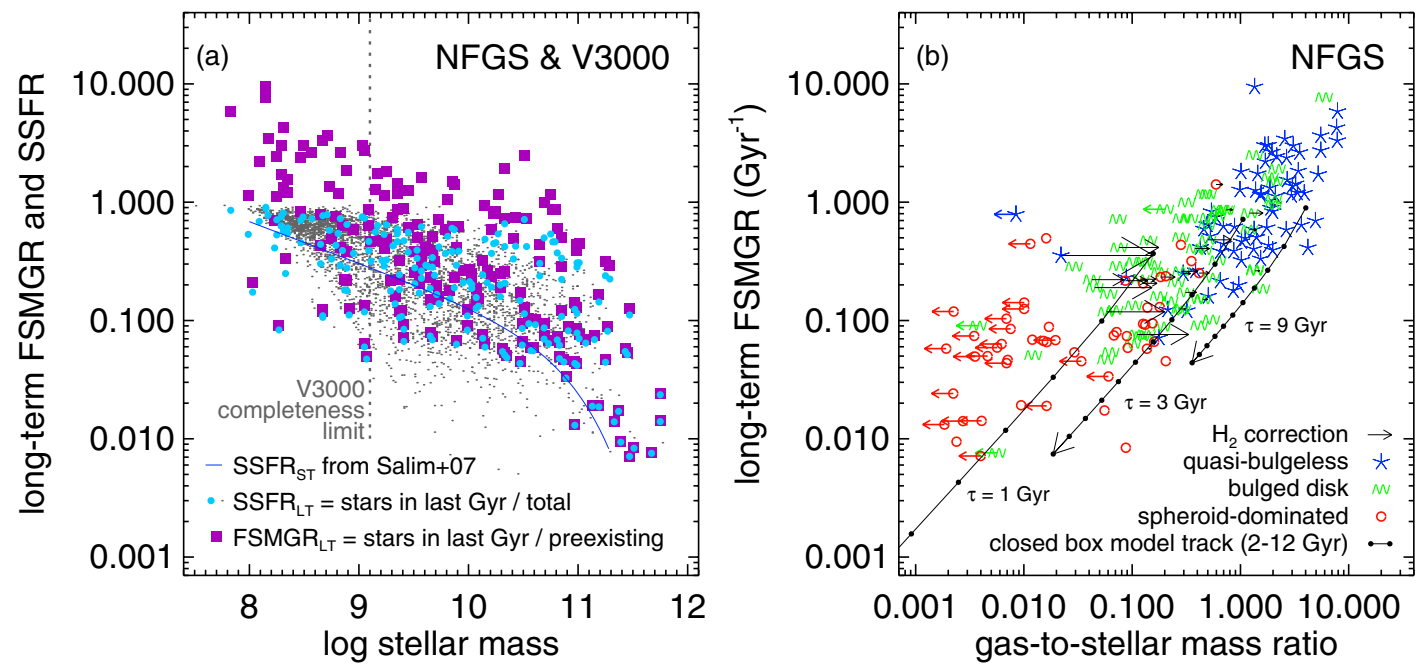

Figure 9. FSMGR $\mathrm{LT}_{\mathrm{LT}}$ as a function of stellar mass and $M_{\mathrm{H}} / M_{*}$. (a) Comparison of FSMGR $\mathrm{LT}_{\mathrm{LT}}$ and SSFR $\mathrm{LT}_{\mathrm{LT}}$ derived from stellar population modeling of NFGS galaxies (large colored symbols) reveals the essential difference between these quantities: FSMGR divides by preexisting rather than by total mass, so it does not asymptote to one over the unit of time at high growth rates. Small background dots represent SSFR $\mathrm{LT}_{\mathrm{L}}$ values for the V3000 sample (down to $M_{r}=-17$, hence incomplete below the $M_{*}$ limit shown; Section 2.2). Comparison of the V3000 and NFGS distributions confirms that the NFGS is broadly representative of the general galaxy population, albeit with overrepresentation of high-SSFR galaxies consistent with its parent survey's $B$-band selection. Comparison of the V3000 distribution with the short-term SSFR trend (blue line) provided by S07 reveals an overall shift toward higher SSFRs in our long-term measurements. While some of this effect may reflect the timescale difference, we suspect that photometry differences are more important: as discussed in Section 2.1.1, our photometry yields significantly bluer $u-r$ colors than the SDSS DR7 catalog photometry used by S07. (b) FSMGR $\mathrm{LT}_{\mathrm{L}}$ and $M_{\mathrm{H}} / M_{*}$ define a nearly one-to-one relation on Gyr timescales (note the identical axis ranges). Closed box model tracks (whose arrows point in the direction of greater age) are inconsistent with the data except for the first few time steps, representing galaxies born $\sim 2-5$ Gyr ago. We interpret these results in terms of cosmic accretion in Sections 4.3 and 4.4. We identify three refueling regimes: (1) a gas-dominated, stellar mass doubling (FSMGR > 1) regime; (2) a transitional regime with growth rates and gas fractions at tens of percentage levels; and (3) a gas-starved, minimal growth regime considered quenched. Symbols indicate $\mu_{\Delta}$ classes, which approximately map to these regimes, with interesting complexity in the transition range (see Section 4.4).

(A color version of this figure is available in the online journal.)

between $(u-J)^{m}$ and FSMGR $\mathrm{LT}_{\mathrm{T}}$ where:

$$
\mathrm{FSMGR}_{\mathrm{LT}}=\frac{\text { mass }_{\text {formedinlastGyr }}}{1 \mathrm{Gyr} \times\left(\text { mass }_{\text {preexisting }}\right)} .
$$

We measure FSMGR $\mathrm{LT}_{\mathrm{LT}}$ as part of the same stellar population modeling used to determine stellar masses (Section 2.1.1). Our model grid is designed to sample FSMGR $\mathrm{LT}_{\mathrm{LT}}$ uniformly in the logarithm, with 13 values from $10^{-3}, 10^{-2.65}, 10^{-2.3}, \ldots$, up to $10^{1.2}$. We estimate both the likelihood-weighted mean and

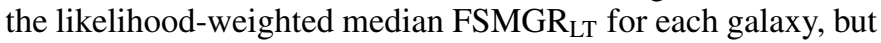
since they are very similar, we plot the means for visual clarity (the medians are discretized by construction).

The definition of FSMGR $\mathrm{LT}_{\mathrm{L}}$ may seem superficially similar to that of a specific star formation rate (star formation rate normalized to current stellar mass), e.g., as traced by $\mathrm{EW}(\mathrm{H} \alpha)$ over short timescales. However, FSMGR $\mathrm{LT}_{\mathrm{LT}}$ is not equivalent to a 
long-term SSFR, because the newly formed stellar mass appears only in the numerator. In contrast, conventional definitions of SSFR (e.g., as in the work of S07) include new stellar mass in both the numerator and the denominator, so that at high growth rates the SSFR cannot exceed one over the unit of time in which the SFR is measured (see Figure 9(a)). Thus, SSFRs offer limited insight into star formation in high fractional growth regimes.

Plotting FSMGR $_{\mathrm{LT}}$ directly against $M_{\mathrm{HI}} / M_{*}$, we find a remarkably linear relationship for star-forming galaxies (Figure 9(b)). It is particularly striking that in per Gyr units, the newly formed-to-preexisting stellar mass ratios for quasibulgeless and bulged disk galaxies are not merely proportional to their gas-to-stellar mass ratios, but instead are almost the same. The numerical coincidence of scales on the FSMGR $\mathrm{LT}_{\mathrm{LT}}$ and $M_{\mathrm{HI}} / M_{*}$ axes has profound implications to be discussed in Section 4.3, so it is worth noting that our estimates of FSMGR LT $_{\text {T }}$ are higher than would be expected from previous work. Specifically, if we convert our FSMGRs to SSFRs (dots in Figure 9(a)), we find that both the V3000 sample and the NFGS lie above the fit line for short-term SSFRs from S07. A small amount of this difference may be due to the short-term versus long-term measurement (see Salim et al. 2009), and the excess of scatter for the NFGS may certainly reflect the blue selection of its parent survey (Jansen et al. 2000b), but the majority of the effect is probably due to differences in photometry: as discussed in Section 2.1.1, our reprocessed SDSS magnitudes yield significantly bluer colors than the SDSS DR7 catalog photometry used by $\mathrm{S} 07$.

\subsection{Interpreting the $M_{\mathrm{HI}} / M_{*}$ versus FSMGR $R_{\mathrm{LT}}$ Relation}

Since gas fuels star formation, it may seem self-evident that FSMGR ${ }_{\mathrm{LT}}$ should correlate with $M_{\mathrm{H}} / M_{*}$. Indeed, Zhang et al. (2009) have argued that the Kennicutt-Schmidt relation can be used to explain the correlation of another long-term star formation tracer, $g-r$ color, with $M_{\mathrm{HI}_{\mathrm{I}}} / M_{*}$. Motivated by the Kennicutt-Schmidt relation, they add $i$-band surface brightness to $g-r$ color to obtain a correlation with $M_{\mathrm{HI}} / M_{*}$ with 0.31 dex scatter, albeit with a less diverse/representative sample than we have presented (following K04, they analyze galaxies with $\mathrm{H}$ I detections in HyperLEDA). Despite the appeal of their straightforward interpretation, there are two reasons to rethink the underpinnings of the photometric gas fraction technique.

First, most astronomers agree that stars form in molecular gas (e.g., Krumholz et al. 2011). Yet U-NIR colors correlate only with atomic gas or the combination of atomic+molecular gas-a plot of U-NIR colors versus $M_{\mathrm{H}_{2}} / M_{*}$ is a scatter plot (red points in Figure 10). A typical plot of the Kennicutt-Schmidt relation would show that the $\mathrm{H} \alpha$-derived star formation rate has a direct correlation with the mass in $\mathrm{H}_{2}$, its immediate precursor, where both are typically divided by surface areas to yield surface densities (e.g., Bigiel et al. 2008; Schruba et al. 2011). The timescales involved in the Kennicutt-Schmidt relation are measured in tens of Myr, so long-lasting $U$-band flux is not an ideal real-time tracer of star formation and molecular gas consumption. In contrast, U-NIR color tracks FSMGR $_{\mathrm{LT}}$ over timescales long enough to register whether molecular gas is resupplied from a larger reservoir. Because $\mathrm{H}$ I can resupply $\mathrm{H}_{2}$ and makes up most of the gas in a typical galaxy $\left(\mathrm{H}_{2}\right.$-rich galaxies are relatively less common and also typically gas-poor; Figures 7(a), 8(a), and 10), H I measurements generally trace a galaxy's potential for long-term molecular gas consumption better than $\mathrm{H}_{2}$ measurements, which reflect only current $\mathrm{H}_{2}$. For the few galaxies in Figure 8(a) with large $\mathrm{H}_{2}$ corrections (green arrows), the $\mathrm{H}_{2}$ represents a large fraction of the total gas reservoir and thus helps tighten the U-NIR versus $M_{\mathrm{gas}} / M_{*}$ relation. Of course, short-term processes that affect $\mathrm{H}_{2}$ and $\mathrm{EW}(\mathrm{H} \alpha)$ also affect U-NIR colors-we see that $\mathrm{EW}(\mathrm{H} \alpha)$ varies with $(u-J)^{m}$ in Figure 10(a) - but the scatter in the plot of $M_{\mathrm{HI}} / M_{*}$ versus $\mathrm{EW}(\mathrm{H} \alpha)$ is $>20 \%$ higher than in the plot of $M_{\mathrm{HI}} / M_{*}$ versus $(u-J)^{m}$ (Figure 10(b) versus Figure 10(a)). The long timescale of U-NIR colors would seem optimal for predicting $M_{\mathrm{H}} / M_{*}$, implying that the underlying physics is distinct from the short timescale physics driving the Kennicutt-Schmidt relation.

Moreover, the long U-NIR timescale suggests a second level of reinterpretation. We have shown that U-NIR colors reflect FSMGR $_{\mathrm{LT}}$ past-averaged over a Gyr (Section 4.2). Why such long-term past-averaged star formation should correlate tightly with present gas richness is not obvious in a "gas reservoir causes star formation" picture. One might suppose that the correlation could result from a well-behaved time lag between $M_{\mathrm{HI}} / M_{*}$ and $\mathrm{FSMGR}_{\mathrm{LT}}$ in a closed box scenario, but again we note the surprising fact that these quantities are not just proportional, but nearly the same when star formation is integrated over the last Gyr. This fact, along with the modest scatter for non-gasstarved galaxies in Figure 9(b), rules out any reasonable closed box model. To illustrate the difficulty, we show three model tracks in Figure 9(b), with gas depletion times of 1, 3, and 9 Gyr. The tracks obey pure closed box evolution:

$$
\begin{gathered}
M_{\mathrm{gas}} / M_{*}=\frac{e^{-t / \tau}}{1-e^{-t / \tau}}, \\
\mathrm{FSMGR}_{\mathrm{LT}}=\frac{e^{-(t-1) / \tau}-e^{-t / \tau}}{1-e^{-(t-1) / \tau}},
\end{gathered}
$$

where $\tau$ is measured in Gyr. Intersection with the data requires an implausibly young box (just a few Gyr), which should ideally also have short gas depletion time.

The most natural way out of this cause-effect conundrum is to suppose that most galaxies are routinely refueled, both in the sense of external gas accretion and in the sense of internal gas transport and $\mathrm{H}$ I-to- $\mathrm{H}_{2}$ conversion. In this view, the coincidence of axes in Figure 9(b) implies that the entire cold gas reservoir of an unquenched galaxy is routinely turned into stars and fully replenished over the time span of a Gyr, within a factor of a few. It follows that Gyr timescales must be longer than the typical time intervals between any discrete processes necessary to maintain refueling and star formation, such as gas accretion events, development of internal instabilities, and/or interactions with companions. Thus, the fundamental physics underlying the $M_{\mathrm{HI}} / M_{*}$ and FSMGR $\mathrm{LT}_{\mathrm{LT}}$ relation involves gas refueling on roughly Gyr timescales.

\subsection{Refueling Regimes}

From a refueling perspective, three regimes emerge naturally from Figure 9(b), reflecting coordinated shifts in gas richness, growth rate, and morphology (as parameterized by $\mu_{\Delta}$ class in Figures 8 and 9). The accretion-dominated regime of gas-dominated quasi-bulgeless disks is characterized by extremely rapid growth $\left(\mathrm{FSMGR}_{\mathrm{LT}} \sim 1\right.$ implies stellar mass doubling on Gyr timescales) and ultra-blue colors. The processing-dominated regime of "normal" bulged disks is mostly characterized by moderate growth and gas content at tens of percentage levels, but these transitional systems show greater diversity in color and gas content than quasi-bulgeless or spheroid-dominated systems (Figure 11). We will argue in 


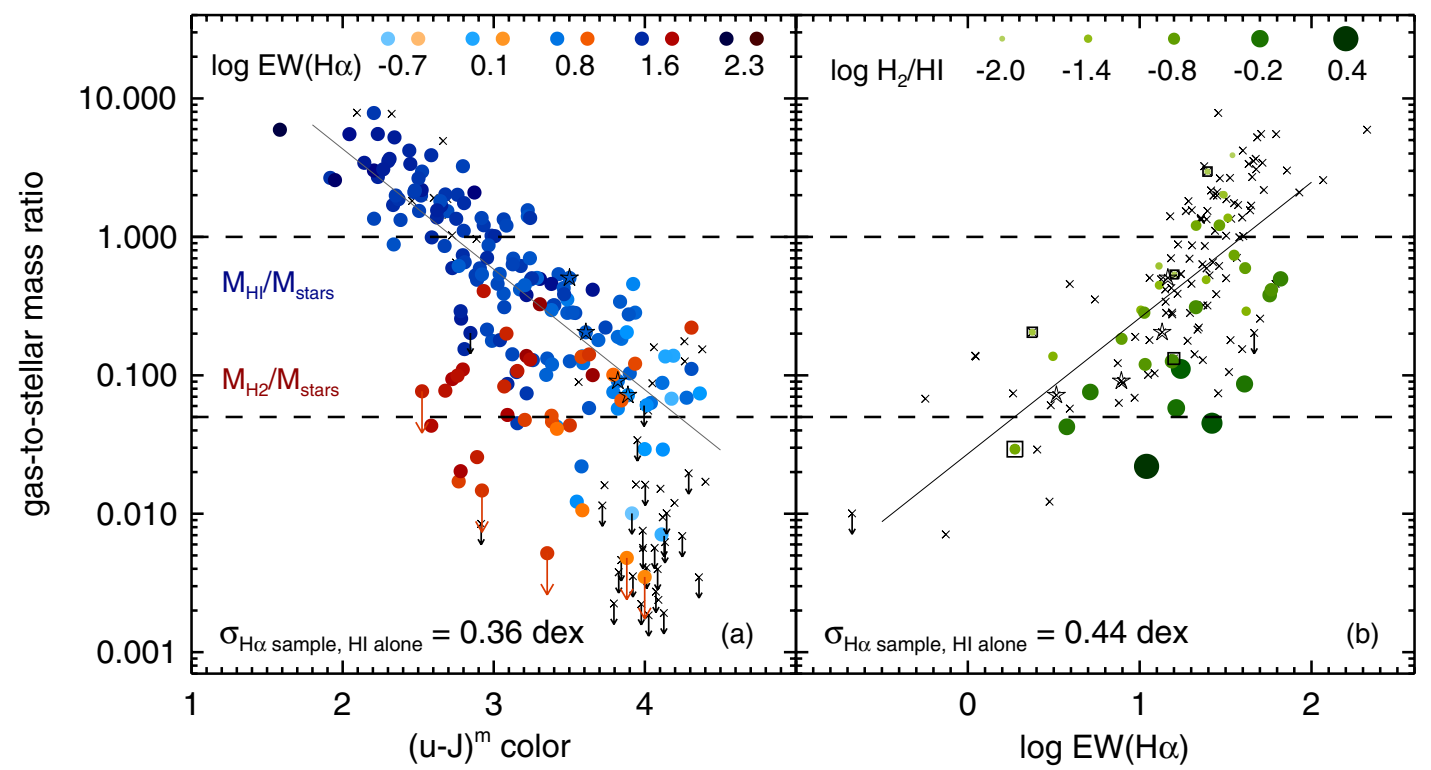

Figure 10. Relationship of gas-to-stellar mass ratios to long-term and short-term 〈SSFR〉, as parameterized by U-NIR color and EW(H $\alpha$ ), respectively, for the NFGS. (a) $M_{\mathrm{HI}} / M_{*}$ (blue) and $M_{\mathrm{H} 2} / M_{*}$ (red) vs. $(u-J)^{m}$ color as in Figure 8(a), with points coded by EW(H $\left.\alpha\right)$ as shown in the legend ( $\times$ symbols mark galaxies either lacking $\mathrm{H} \alpha$ data or undetected in $\mathrm{H} \alpha$ ). Downward arrows indicate upper limits. Stars mark AGNs as classified by Jansen et al. (2000a). (b) $M_{\mathrm{H}_{\mathrm{I}}} / M_{*}$ vs. EW(H $\alpha$ ), with points coded by $\mathrm{H}_{2} / \mathrm{H}$ I mass ratio as shown in the legend. Additional symbols are as in panel (a), except now $\times$ symbols mark galaxies lacking $\mathrm{H}_{2}$ data and boxed points denote $\mathrm{H}_{2}$ upper limits. The scatter in panel (b) is notably higher than in panel (a), relative to fits restricted to $\mathrm{H} \alpha$-detected galaxies in both panels.

(A color version of this figure is available in the online journal.)
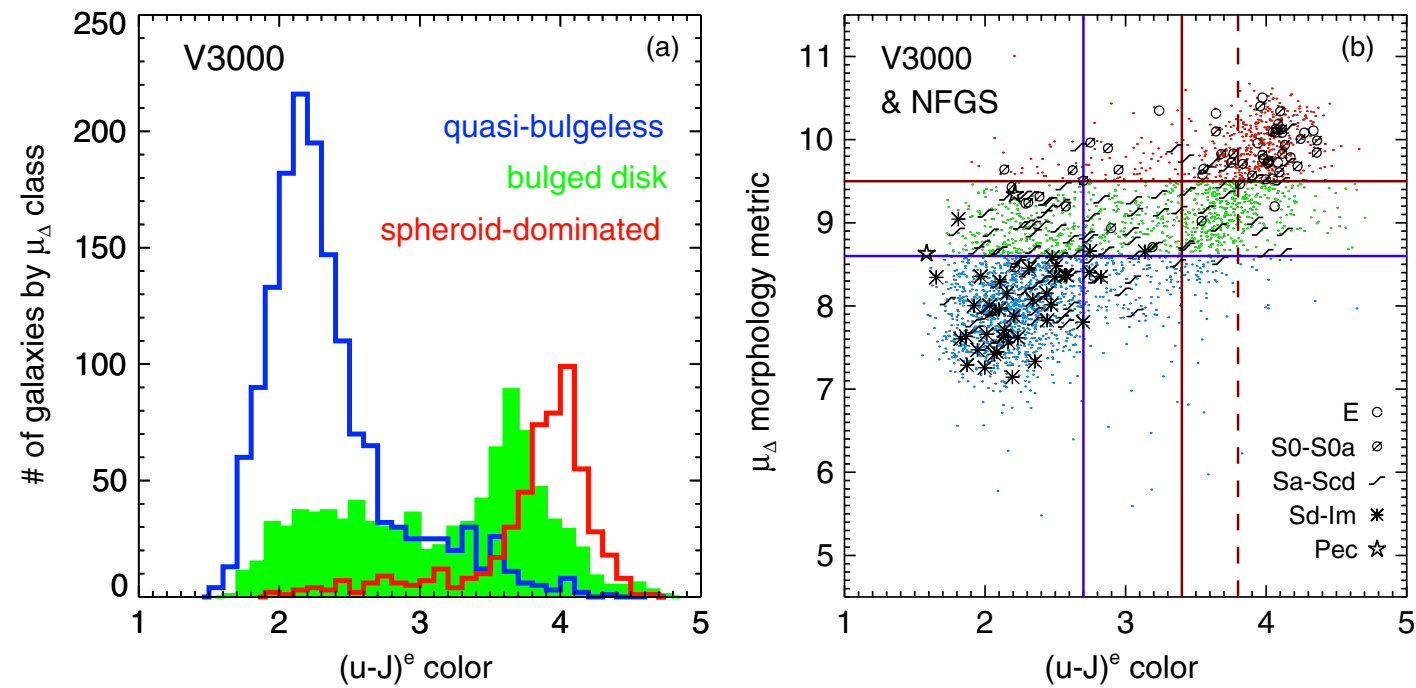

Figure 11. Galaxy morphology in relation to de-extincted $(u-J)^{e}$ color, using $\mu_{\Delta}$ classes for the V3000 sample $\left(M_{r}<-17.73\right)$ and traditional morphological types for the NFGS. Horizontal lines in panel (b) mark the divisions used to define the three $\mu_{\Delta}$ classes (see Figure 1), while vertical lines mark shifts in their relative dominance as seen in panel (a). Ultra-blue fast-growing quasi-bulgeless galaxies and red-and-dead spheroid-dominated galaxies occupy distinct loci. Bulged disk galaxies are transitional, displaying a broad range of colors, but also show a clear peak starting at $(u-J)^{e} \sim 3.4$, just shy of the quenched spheroid regime. Visual inspection of galaxies in this peak reveals residually star-forming S0 or dying spiral galaxies.

(A color version of this figure is available in the online journal.)

Section 4.4.3 that this regime may also be considered to include blue spheroid-dominated galaxies, to the extent that they are likely to regrow disks rather than quench. Finally, the quenched regime of red-and-dead spheroid-dominated galaxies is characterized by gas poverty $\left(M_{\mathrm{HI}} / M_{*} \lesssim 0.05\right)$ and slow growth $\left(\right.$ FSMGR $\left._{\mathrm{LT}} \lesssim 0.1\right)$, although the exact definition of this regime is debatable, given the existence of a distinct population of nearly quenched bulged-disk systems with $(u-J)^{e}=3.4-3.8$ as seen in Figure 11.

Below we examine the significance of these regimes in the context of a broader picture of galaxy evolution. We first relate regime transitions to the threshold and bimodality scales reviewed in Section 3, with attention to the complicating factor of environment, and then go on to discuss implications of the refueling regime picture for galaxies typical of each regime.

\subsubsection{Regime Transitions and Galaxy and Halo Mass Scales}

The existence of red disk and blue spheroid crossover systems in Figure 11 points to the complexity of refueling and quenching, likely reflecting the influence of large-scale environment as well as local galaxy interactions and mergers. Environmental analysis of the V3000 sample and its parent survey will be presented elsewhere (A. J. Moffett et al., in preparation; K. D. Eckert et al., in preparation; S. J. Kannappan et al., in preparation), but 
for the present discussion we note that these studies present a general picture in which galaxies that are central (most massive) in their dark matter halos follow the simplest trajectories from accretion-dominated to quenched, as judged by U-NIR colors, while satellite galaxies display greater diversity, including some of the most extreme crossover states. This picture reinforces that of Peng et al. (2012), who argue that central galaxies experience essentially zero environmentally driven evolution, quenching solely as a function of galaxy mass, while satellites are responsible for all signatures of quenching that increase with group halo mass. Thus, central/satellite differences offer a way to reconcile the scattered dependence of gas-richness on galaxy mass with the existence of clear gas-richness transitions at the threshold and bimodality scales, below and above which, respectively, galaxies typical of the accretion-dominated and quenched regimes become abundant (as seen in Section 3 and further confirmed for the V3000 sample below).

If we assume that observed transitions in gas-richness, morphology, and dynamics at the threshold and bimodality scales reflect the en masse transformation of central galaxies, then we can link these scales to equivalent halo mass scales. At the threshold scale, the central-galaxy $M_{*}$-to- $M_{\text {halo }}$ calibration of Behroozi et al. (2013) indicates that $M_{*} \sim 10^{9.7} M_{\odot}$ corresponds to $M_{\text {halo }} \sim 10^{11.5} M_{\odot}$. Equivalently, the threshold velocity of $\sim 125 \mathrm{~km} \mathrm{~s}^{-1}$ matches a halo virial velocity of $V_{\text {vir }} \sim 100 \mathrm{~km} \mathrm{~s}^{-1}$ if $V_{\text {galaxy }} / V_{\text {vir }} \sim 1.3$, as reported by Reyes et al. (2012). At the bimodality scale, the same logic implies halo parameters of $M_{\text {halo }} \sim 10^{12.1} M_{\odot}$ and $V_{\text {vir }} \sim 160 \mathrm{~km} \mathrm{~s}^{-1}$.

These two halo mass scales are potentially important in relation to cosmic accretion. First, observational analysis suggests that above a halo mass of $\sim 10^{12.1} M_{\odot}$, central galaxy $M_{*}$ rises much more slowly with growing halo mass (Leauthaud et al. 2012), implying that the primary mode of central galaxy growth that operates at lower halo mass is being valved off above $M_{\text {halo }} \sim 10^{12.1} M_{\odot}$ (corresponding to the bimodality scale). Second, several theoretical prescriptions suggest that somewhere in the range $M_{\text {halo }}=10^{11}-10^{12} M_{\odot}$ (likely corresponding to the threshold scale), halo gas cooling becomes much more efficient, such that $\gtrsim 50 \%$ of baryons accreted onto the halo over the age of the universe cool within the same time (Lu et al. 2011). In the specific case of the "cold-mode" accretion picture (Birnboim \& Dekel 2003; Kereš et al. 2005), wherein slow hotmode accretion replaces rapid cold-mode accretion as shock heating becomes more effective with increasing halo mass, calculations by Dekel \& Birnboim (2006, see their Figures 2 and 3) show that at $z=0$, the shock-heating transition occurs in the inner halo at $0.1 \times$ the virial radius for halos with $V_{\text {vir }} \gtrsim 100 \mathrm{~km} \mathrm{~s}^{-1}$ (the threshold scale for centrals), while the transition expands to the outer halo past the virial radius for halos with $V_{\text {vir }} \gtrsim 170 \mathrm{~km} \mathrm{~s}^{-1}$ (the bimodality scale for centrals). Recent work using the moving-mesh AREPO code questions the details of this picture, as about half of simulated cold-mode accretion may reflect numerical artifacts (Nelson et al. 2013), yet overall accretion rates onto halos are actually higher using AREPO, due to faster cooling of the hot mode, and the general halo mass dependence of cooling remains. Furthermore, we note that the onset of a "rapid" accretion mode when the cooling radius exceeds the halo virial radius is a common feature of many semi-analytic cooling prescriptions, as reviewed by $\mathrm{Lu}$ et al. (2011), most of which do not involve a cold-mode/hot-mode distinction per se.

In this context, it is interesting to revisit the possibility of a sharp transition at the threshold scale as proposed by Dalcanton et al. (2004), which might constrain theoretical models of accretion. To construct a statistically robust sample to test this possibility, we first estimate baryonic masses for the full V3000 sample down to $M_{r}=-17$. We adopt the photometric gas fraction estimator presented in Figure 8(a) for galaxies with $(u-J)^{m}<3.7$ :

$$
\log M_{\mathrm{HI}} / M_{*}=2.70-0.98(u-J)^{m}
$$

with 0.34 dex scatter. We assign gas masses using Monte Carlo methods to produce realistic variations matching this $0.34 \mathrm{dex}$ scatter in $M_{\mathrm{HI}} / M_{*}$, while not allowing gas masses higher than the measured upper limits. For galaxies redder than $(u-J)^{m}=$ 3.7, we assign gas fractions randomly in the logarithmic interval from $M_{\mathrm{HI}} / M_{*}=0.001$ to the limit value or 0.5 , whichever is smaller. Finally, we sub-select a baryonic mass-limited sample from the V3000 data set with $M_{\text {bary }}>10^{9.3} M_{\odot}$ (the expected completeness limit; see Section 2.2). The $M_{\mathrm{HI}} / M_{*}$ versus $V$ distribution for this sample is plotted in Figure 12(a). Note that the offset in $V$ between detections and limit replacements is due to the fact that most detections can scatter to low or high $V$, whereas limits (and some detections, e.g., those with $i<50 \mathrm{deg}$ ) must be plotted with an inferred $V$ from the all-galaxy $M_{r}-V$ relation, which tends to overestimate $V$ for gas-poor galaxies (Figure 6).

Based on this analysis, Figure 12(a) shows no obviously sudden onset of gas-richness for the V3000 sample. We do see that gas-dominated galaxies become the majority population roughly below the threshold scale (histograms at the top of Figure 12(a)), and that the V3000 sample shows the same large, strongly mass-dependent scatter in $M_{\mathrm{H}} / M_{*}$ that we saw for the NFGS in Figure 7, lending credence to the NFGS results. Gasstarved dwarfs are now represented (based on $\left.(u-J)^{m}\right)$ but drop sharply in relative frequency as gas-dominated galaxies become more common. Furthermore, the gas-starved dwarf population resides predominantly in the highest-density environments seen in Figure 4, such as the Coma Cluster, implying environmental quenching. By contrast, the majority of dwarf galaxies are isolated and gas-rich.

\subsubsection{The Accretion-dominated Regime}

For the quasi-bulgeless, gas-dominated disks typical of the accretion regime, fresh gas is accreted as fast as or faster than it can be consumed, based on Figure 9(b). Most quasibulgeless disks scatter around FSMGR $\mathrm{FT}_{\mathrm{LT}} \sim 1 \mathrm{Gyr}^{-1}$, suggesting that they are capable of doubling their stellar masses each Gyr. Thus, typical gas-dominated Sd-Im galaxies are growing exponentially, at least in the "high-mass dwarf" regime we explore (Bouché et al. 2010 note the possibility of an accretion "floor" that could prevent effective gas cooling for lower-mass galaxies). This conclusion is consistent with that of Moster et al. (2013), who infer from a multi-epoch halo abundance matching analysis that today's low-mass galaxies are still increasing in star formation rate (where these authors' definition of low mass roughly equates to below the threshold scale).

The standard counterargument supposes that dwarf galaxies have intrinsically bursty star formation and therefore experience off states that ensure low time-averaged SSFR despite high short-term SSFR (e.g., Feulner et al. 2005). However, we have measured high growth rates averaged over the last Gyr. Moreover, systematic searches for non-star-forming dwarf galaxies have shown them to be exceedingly rare in field environments 

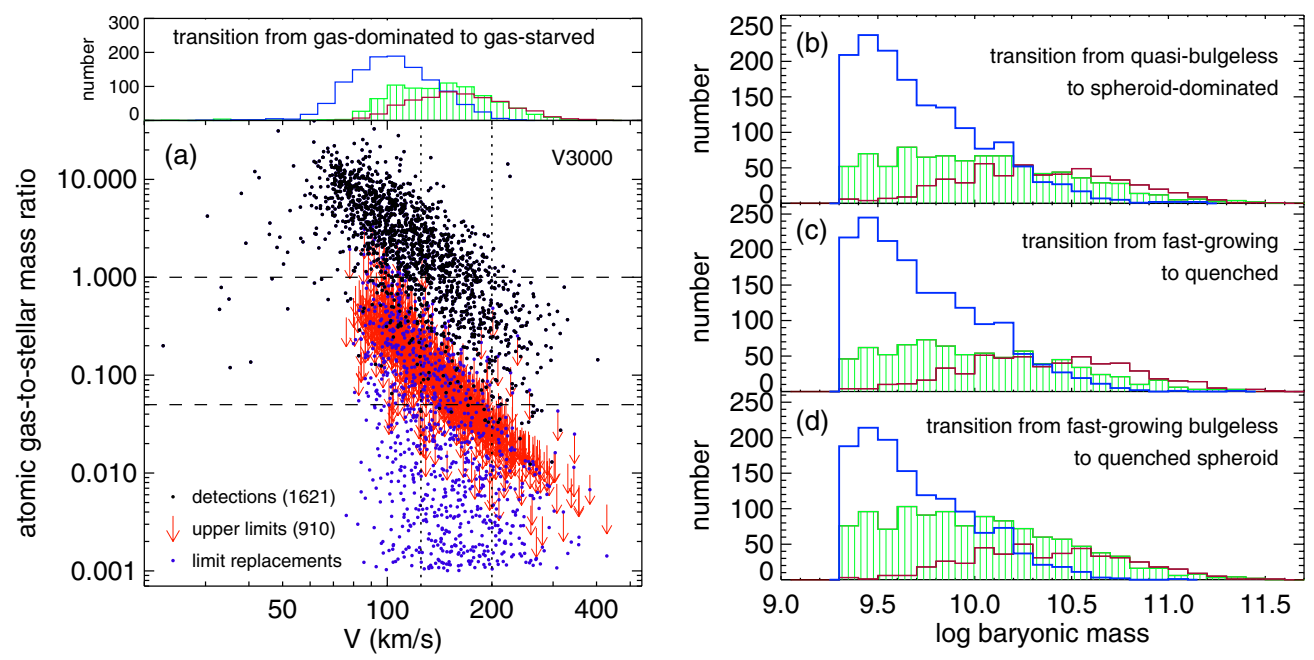

Figure 12. (a) $M_{\mathrm{H}_{\mathrm{I}}} / M_{*}$ vs. $V$ for the $\mathrm{V} 3000$ sample, using the photometric gas fraction technique to replace upper limits with likely values based on $(u-J)^{m}$ color (see Section 4.4.1 for details). We now draw from the full V3000 sample down to $M_{r}=-17$ to identify galaxies with measured or estimated $M_{\text {bary }}>10^{9.3} M_{\odot}$, so the absence of quenched galaxies at low $M_{\text {bary }}$ is plausibly correct (see Section 4.4.2 for further discussion). The offset in $V$ between detections and limits is not significant but reflects the use of the all-galaxy $M_{r}-V$ relation to provide $V$ for limits (Section 2.2), which suppresses natural scatter. Vertical lines mark the threshold and bimodality scales, and horizontal lines divide the gas-dominated, intermediate-gas, and gas-starved regimes. Histograms of these regimes (blue, green, and red, respectively) are shown at the top of the panel, illustrating their relationship to the threshold and bimodality scales. (b)-(d) Mass distributions of galaxies in different color and morphology regimes for the baryonic mass limited sample shown in panel (a). We consider three ways of identifying the transitional regime (green histogram) with reference to Figure 11. In panel (b), transitional galaxies are those in the bulged disk $\mu_{\Delta}$ class. In panel (c), transitional galaxies are those whose colors fall in the range $(u-J)^{e}<2.7-3.8$, which is broadly inclusive of residually star-forming galaxies at the red end. In panel (d), transitional galaxies are all those that do not occupy the regions of $(u-J)^{e}$ vs. $\mu_{\Delta}$ parameter space dominated by blue quasi-bulgeless systems $\left((u-J)^{e}<2.7, \mu_{\Delta}<8.6\right)$ or quenched spheroids $\left((u-J)^{e}>3.4\right.$, $\mu_{\Delta}>9.5$ ). Only by this hybrid definition does the transitional population become "typical" (in the sense of "more common than either other population considered separately") within the range between the threshold and bimodality scales.

(A color version of this figure is available in the online journal.)

(Lee et al. 2011; see also Lee et al. 2009) and, when found, to be associated with proximity to massive hosts (Geha et al. 2012). In the volume-limited V3000 sample, the sharply dropping relative frequency of gas-starved galaxies with decreasing galaxy mass in Figure 12 is likewise consistent with few (or brief) truly off states, although we remind the reader that this sample suffers from residual bias against low surface brightness dwarfs, which might in principle include a high mass-to-light ratio population (Section 2.2). Nonetheless, from the evidence in hand it is reasonable to conclude that most low-mass galaxies achieve high FSMGR $\mathrm{LT}_{\mathrm{TT}}$ through sustained growth.

A related concern is the effect of outflows on dwarfs. However, the early idea that supernova feedback in dwarfs should expel their gas and turn them off (Dekel \& Silk 1986) appears most relevant to galaxies much less massive than we consider, with $V \sim 30 \mathrm{~km} \mathrm{~s}^{-1}$ (Mac Low \& Ferrara 1999). Powerful outflows are indeed expected - the data of Martin et al. (2012) reveal a significant increase in the frequency of strong outflows for $z=0.4-1.4$ galaxies with short-term SSFRs $\gtrsim 0.8 \mathrm{Gyr}^{-1}$ — but they are likely to regulate rather than terminate star formation (see the comparison of outflow scenarios in Davé et al. 2013). The cosmological simulations of Brooks et al. (2007) also provide another perspective, suggesting that even significant outflows are less important than low star formation efficiency in setting the gas and metal content of low-mass galaxies. Of course, "low" star formation efficiency in systems experiencing cosmic accretion may not imply low SSFRs in absolute terms, but simply star formation that cannot keep up with the inflow rate.

As noted by Khochfar \& Silk (2011) in the course of interpreting high-redshift observations, galaxies with sustained high SSFRs may pose a challenge for current models of galaxy evolution. For example, the simulation-motivated analytic model of
Davé et al. (2012), which assumes an equilibrium between mass inflow, mass outflow, and star formation, would predict SSFRs reaching only $\sim 0.2-0.3 \mathrm{Gyr}^{-1}$ for isolated $z=0$ galaxies near the threshold scale (in rough agreement with the simulations of van de Voort et al. 2011, in which feedback prevents accretion from reaching the ISM for such galaxies). In this context, it is interesting to consider the possibility of excess accretion, leading to non-equilibrium "puddling" of ionized gas, contrary to the assumptions of Davé et al. (2012). While we have argued that cold $\mathrm{H} \mathrm{I}+\mathrm{H}_{2}$ gas cycles through galaxies on Gyr timescales, nothing in Figure 9 requires that ionized gas arrive at the same rate. In fact, analyses of the baryonic Tully-Fisher relation suggest that dwarf galaxies may contain three to four times larger gas reservoirs than their $\mathrm{HI}$-derived gas masses imply (Pfenniger \& Revaz 2005; Begum et al. 2008). Our own baryonic $M-V$ relation in Figure 6 may be "missing" such a multiplier, which would straighten it out below $V \sim 125 \mathrm{~km} \mathrm{~s}^{-1} .^{23}$

Any such excess gas would presumably end up being processed intermittently in merger-driven bursts (distinct from internally driven bursts as considered above), likely producing phenomena such as blue compact dwarfs (BCDs). BCDs have previously been linked to gas-rich dwarf-dwarf mergers (e.g., Pustilnik et al. 2001; Bekki 2008). If accretion rates remain high after such events, the remnants may enjoy rapid disk regrowth. For example, the BCD NGC 7077 (shown in Figure 7 at $M_{\mathrm{HI}} / M_{*} \sim 0.4$ and $V \sim 60 \mathrm{~km} \mathrm{~s}^{-1}$ and classed as an S0a in the NFGS) reveals a combination of color gradients, molecular and atomic gas content, and GALEX-detected extended UV disk structure that together suggest that this galaxy is a post-merger

\footnotetext{
${ }^{23}$ Another interesting set of results in the literature centers on the possibility of substantial CO-dark molecular gas in dwarf galaxies (e.g., Madden et al. 1997). If such undetected $\mathrm{H}_{2}$ gas were comparable in mass to the $\mathrm{H} \mathrm{I}$, it might explain some of the scatter in $M_{\mathrm{HI}} / M_{*}$ versus FSMGR $\mathrm{LT}_{\text {. }}$.
} 
system just starting to rebuild its disk (Moffett et al. 2012; S13). If such a galaxy enters a reduced accretion regime post-merger, it may give rise to an $\mathrm{Sa}-\mathrm{Sc}$ spiral, while if it stays in a high accretion regime, it may soon revert to Sd-Im morphology. Consistent with this picture, kinematic signatures of past mergers are observed in the stellar components of even very late-type dwarf disks (e.g., counterrotating stars and/or thick disks; Kannappan \& Fabricant 2001; Yoachim \& Dalcanton 2005; Leaman et al. 2009, 2012).

The reader might reasonably object to the notion of exponentially growing $\mathrm{Sd}-\mathrm{Im}$ galaxies based on the implausibility of efficient gas processing in such systems (although the rate of molecular gas conversion to stars in such galaxies may actually be higher than in spirals, e.g., Gardan et al. 2007; Pelupessy \& Papadopoulos 2009). However, we stress that regardless of any deficiency in gas transport mechanisms, the fact that FSMGR LT $_{\mathrm{LT}}$ values remain high in the accretion-dominated regime implies that accretion-dominated systems do not have to form stars efficiently in order to form them rapidly: the rate of gas influx is apparently sufficient to overwhelm any inefficiency in consumption. Moreover, it is not obvious that the conventional definition of star formation efficiency, i.e., the inverse of the timescale for the current rate of star formation to consume the current reservoir of gas, makes sense for accretion-dominated galaxies. Huang et al. (2012) report as a paradox the fact that the most gas-rich galaxies in ALFALFA have both the highest SSFRs and the lowest star formation efficiencies, and they attribute this paradox to a bottleneck in processing the gas. We would propose that the paradox arises from the fact that the English words "low efficiency" connote galaxies lazily consuming gas: if instead the gas is pouring in faster than even the highest-SSFR galaxies can possibly consume it, such galaxies might be better termed "overwhelmed" than "inefficient."

\subsubsection{The Processing-dominated Regime}

For galaxies in the processing-dominated regime, gas is still consumed roughly as fast as it is accreted (Figure 9(b), in agreement with Fraternali \& Tomassetti 2012). For centrals whose halos are experiencing reduced cosmic accretion, the transition to a processing-dominated state may be self-reinforcing. Slowed dilution of the ISM with fresh gas and/or reduced metal loss in outflows may lead to more efficient $\mathrm{H}_{2}$ formation on dust grains (Krumholz \& Dekel 2012). This process may in turn increase consumption efficiency for the diminished infalling gas and accelerate the galaxy's evolution to a processing-dominated state (thus decelerating growth). In addition, mild quenching may encourage more prominent stellar bulges and more concentrated disks that further promote higher gas densities and more efficient star formation (e.g., Blitz \& Rosolowsky 2006).

Notably, galaxies in the processing-dominated regime show deviations in $M_{\mathrm{HI}} / M_{*}$ that seem to correlate with high $\mathrm{H}_{2} / \mathrm{HI}$, based on the incomplete $\mathrm{H}_{2}$ data available (Figure 9). Our previous analysis of NFGS galaxies has revealed bursty, correlated variations in central and outer disk colors and $\mathrm{H}_{2}$ / $\mathrm{H}$ I ratios, particularly for bulged disks (S13). These variations seem to reflect externally triggered gas inflow events and central star formation enhancements, with corresponding lulls in outerdisk growth (Kannappan et al. 2004; S13). The most extreme events lead to the formation of blue-sequence E/S0s, which account for many of the spheroid-dominated galaxies embedded in the main FSMGR $\mathrm{LT}-M_{\mathrm{H}} / M_{*}$ locus in Figure 9. Based on a variety of evidence, including identification of temporal sequences in a "fueling diagram" linking stellar populations and gas content, we have argued elsewhere that blue-sequence $\mathrm{E} / \mathrm{S} 0$ s reflect a process of gas-rich merger-induced bulge building followed by disk regrowth, which may play a crucial role in the morphological evolution of disk galaxies (KGB; W10a; Wei et al. 2010b; Moffett et al. 2012; S13). Other authors have also documented a "living" population of E/S0s (e.g., Driver et al. 2007; Cortese \& Hughes 2009; Thilker et al. 2010; HuertasCompany et al. 2010; Marino et al. 2011). In the mass regime below the threshold scale, blue-sequence E/S0s overlap with $\mathrm{BCDs}$, suggesting a continuity of phenomena; such galaxies are most common below the threshold scale, with a tapering population up to the bimodality scale.

While bursty star formation has traditionally been ascribed to dwarf galaxies, Kauffmann et al. (2006) demonstrate that stochastic star formation is in fact most prevalent for galaxies with the intermediate masses and surface mass densities of bulged spirals (which happen to coincide with those of bluesequence E/SOs; KGB). From a comparison to semi-analytic models, these authors infer that the observed stochasticity reflects efficient processing of fresh gas infall. Ferreras et al. (2004) advance similar ideas in their analysis of intermediateredshift galaxies, concluding that efficient, short-lived bursts are typical for galaxy rotation velocities $V \gtrsim 140 \mathrm{~km} \mathrm{~s}^{-1}$. These authors' bursty accretion picture is roughly consistent with ours, with the caveat that we emphasize the roles of not only cosmic infall but also gas processing mechanisms in refueling. The fundamental importance of both is illustrated by an "exception that proves the rule": the gas-dominated S0 at $V \sim 90 \mathrm{~km} \mathrm{~s}^{-1}$ and $M_{\mathrm{HI}} / M_{*} \sim 4$ in Figure 7 (a) is UGC 9562, a polar ring galaxy for which the misalignment of fresh accretion relative to the inner bulge/disk orientation likely inhibits normal gas transport, creating a system hovering between the accretiondominated and processing-dominated regimes. ${ }^{24}$

Both externally driven and secular gas inflow mechanisms should become more important above the threshold scale. Hopkins et al. (2010) perform an analytic timescale analysis comparing growth by cosmic accretion, merging, and secular disk evolution as a function of galaxy mass at $z=0$. They find that although merger rates are insignificant compared to gas accretion rates for $M_{\text {bary }} \lesssim 10^{10} M_{\odot}$, the two become comparable at intermediate masses, as the merger rate (per Gyr) increases with galaxy mass (e.g., Maller et al. 2006). Furthermore, Sinha \& Holley-Bockelmann (2012) find that the flyby interaction rate, normally ignored in theoretical merger histories, may exceed the minor merger rate at intermediate-mass scales. At these mass scales Hopkins et al. also find that reduced accretion enables secular processes such as bar formation to become relatively more important. Observationally, bars appear more common in lower-mass, later-type galaxies than in Sa-Sc galaxies (Barazza et al. 2008; Nair \& Abraham 2010), but Ellison et al. (2011) argue that strong bars in galaxies with $M_{*}>10^{10} M_{\odot}$ may be substantially more effective in enhancing star formation than bars in lower-mass galaxies (although the possibility of differential time lags in enhanced star formation complicates interpretation). On the other hand, central star formation enhancements strong enough to create blue-centered color gradients do not obviously correlate with the presence of

\footnotetext{
24 Cox et al. (2001) make the case that the gas in this polar ring was accreted long before the galaxy's interaction with a neighbor. Independent evidence linking other polar rings to cosmic gas accretion includes low gas-phase metallicity (Spavone et al. 2010) and alignment within a large-scale cosmic wall (Stanonik et al. 2009).
} 
bars, but instead with signs of minor mergers and interactions (Kannappan et al. 2004; Gonzalez-Perez et al. 2011; S13).

While morphology correlates better with FSMGR or U-NIR color $^{25}$ than with mass (compare Figures 11(a) and 12(b); see also Franx et al. 2008), the definition of the processingdominated regime by morphology and/or U-NIR color is complicated by the variety of transitional states. The histograms in Figures 12(b)-(d) show three possible definitions, with only the most inclusive $((\mathrm{d})$ : all bulged disks + bluish spheroiddominated galaxies + reddish quasi-bulgeless galaxies) yielding a numerically dominant population between the threshold and bimodality scales, where intermediate gas-richness systems are the norm (Figure 12(a)). At the red end, this population includes residually star-forming S0s and dying spirals (Figure 11(a)), not unreasonably since such galaxies appear to extend the gas-star formation relation of "normal" Sa-Sc spirals in Figure 8. On the other hand, this analysis suggests that such "normal" spirals, although broad in their mass distribution, are not actually typical at any mass.

\subsubsection{The Quenched Regime}

Most galaxies in the quenched regime have negligible

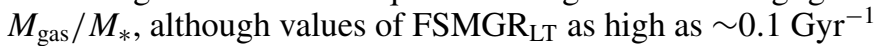
are not uncommon. The low gas-to-stellar mass ratios in this regime may reflect not only slowed cosmic accretion but also ram-pressure stripping and/or efficient AGN feedback in hotgas-dominated halos (e.g., Somerville et al. 2008; Woo et al. 2013). However, significant growth largely uncorrelated with cosmic gas accretion may be possible via intermittent satellite mergers, which might rejuvenate the galaxy either by briefly fueling star formation or by injecting stellar mass formed relatively recently in the satellite (Morganti et al. 2006; Martini et al. 2013). For spheroid-dominated galaxies, Martig et al. (2009) argue that "morphological quenching" inhibits the gas processing necessary to form stars, and indeed, the most massive $\left(V \gtrsim 325 \mathrm{~km} \mathrm{~s}^{-1}\right.$ ) early-type galaxies seem to lack recent star formation regardless of gas content (Serra et al. 2008). Consistent with this idea, we see two degrees of quenching in Figure 11(a), with a distinct population of nearly quenched bulged disks forming a peak just blueward of the red-and-dead peak for spheroid-dominated galaxies. Arguably, these systems represent the extreme tail of the processing-dominated regime, appearing as dying spirals or slightly blue S0s. Previous studies have shown that residual star formation is common for S0s over a wide range in mass (e.g., Salim \& Rich 2010; Moffett et al. 2012), even in environments where Es are quenched (Salim et al. 2012). Stellar mass loss might account for much of the gas supply (Sage \& Welch 2006; Leitner \& Kravtsov 2011). The phenomenon of partial quenching seen in Figure 11 reaffirms that both accretion and processing are important to refueling: although the hot gas in galaxy clusters greatly slows accretion (and also removes gas by ram-pressure stripping), still gas buildup can occur, and disk galaxies remain capable of processing it.

\section{SUMMARY AND CONCLUSIONS}

Our analysis has made use of two samples, both broadly representative of the general galaxy population down to the "high-mass dwarf" regime (baryonic masses $\sim 10^{9} M_{\odot}$ ). These

\footnotetext{
25 Interestingly, the bimodality in $\mu_{\Delta}$ as a function of $(u-J)^{e}$ is much less

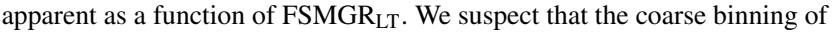
possible FSMGR $\mathrm{LT}_{\mathrm{T}}$ values in our stellar population models blurs the structure seen in $(u-J)^{e}$.
}

samples span a wide range of void to cluster environments, with non-cluster galaxies naturally predominant. The NFGS comprises $\sim 200$ galaxies with newly complete H I data, partial $\mathrm{CO}$ data, and internal kinematic data homogenized from a mix of stellar, ionized gas, and neutral gas measurements. The NFGS $\mathrm{HI}_{\mathrm{I}}$ database is uniquely powerful in providing detections or strong upper limits $\left(M_{\mathrm{H}_{\mathrm{I}}} \lesssim 0.1 M_{*}\right)$ for all galaxies. The V3000 sample comprises $\sim 3000$ galaxies in a volume-limited union of the SDSS and the blind $21 \mathrm{~cm}$ ALFALFA survey, with $\mathrm{H}_{\mathrm{I}}$ upper limits computed for all ALFALFA non-detections. We have combined SDSS and ALFALFA redshifts with redshifts from several other surveys to achieve high completeness in the V3000 sample down to baryonic masses $\sim 10^{9.3} M_{\odot}$, although residual incompleteness for dwarf galaxies remains.

Using these data sets, we have confirmed and clarified observed shifts in gas-richness and morphology at two key galaxy mass scales.

1. Below the gas-richness threshold scale $\left(V \sim 125 \mathrm{~km} \mathrm{~s}^{-1}\right.$; baryonic mass $\sim 10^{9.9} M_{\odot}$ ), gas-dominated quasi-bulgeless disks become most numerous in the galaxy population.

2. Above the bimodality scale $\left(V \sim 200 \mathrm{~km} \mathrm{~s}^{-1}\right.$; baryonic mass $\sim 10^{10.6} M_{\odot}$ ), gas-starved spheroid-dominated galaxies rise to prominence, with numbers comparable to nearly quenched bulged disks.

Notwithstanding these transitions, we have shown that both of our samples display far greater scatter in $M_{\mathrm{gas}} / M_{*}$ as a function of $V$ (a non-covariant mass proxy) than has been commonly assumed. In particular, the NFGS reveals the natural diversity of gas-richness in a sample unbiased with respect to $21 \mathrm{~cm}$ flux: its $M_{\text {gas }} / M_{*}$ versus $V$ plot is striking in its complexity, with quenched galaxies at all masses and scatter $\gtrsim 0.5 \mathrm{dex}$ even for unquenched galaxies. We suggest that the diversity of $M_{\mathrm{gas}} / M_{*}$ has been suppressed in previous studies by selection and detection biases, as well as the practice of plotting $M_{\text {gas }} / M_{*}$ versus $M_{*}$, which allows large covariant errors in $M_{*}$ to enhance the apparent correlation.

In contrast to its complicated correlation with mass, $M_{\mathrm{gas}} / M_{*}$ shows a simple and clean correlation with another fundamental quantity: the long-term fractional stellar mass growth rate $\left(\right.$ FSMGR $\left._{\mathrm{LT}}\right)$, which we define as the ratio of a galaxy's stellar mass formed within the last Gyr to its preexisting stellar mass. The $M_{\mathrm{gas}} / M_{*}-\mathrm{FSMGR}_{\mathrm{LT}}$ correlation represents the physical underpinnings of the remarkably tight ( $\sigma \sim 0.3 \mathrm{dex})$ correlation between $M_{\mathrm{gas}} / M_{*}$ and U-NIR color, used in the "photometric gas fraction" technique of K04, because U-NIR color directly predicts $\mathrm{FSMGR}_{\mathrm{LT}}$. Importantly, a fractional growth rate is related to, but distinct from, an SSFR, as it can exceed a value of one over the unit of time. Choosing Gyr as our unit of time reveals the remarkable fact that for an unquenched galaxy, the mass of new stars formed in the last Gyr is roughly equal to the mass of fresh gas it has available for future star formation, within factors of a few. Thus, for galaxies, past performance is a predictor of future success. We have demonstrated that this result is incompatible with any reasonable closed box model and does not derive from the inherently short-term "gas fuels star formation" physics of the Kennicutt-Schmidt Law, contrary to previous interpretations of the photometric gas fraction technique. Instead, we have argued that it reflects the physics of refueling: both cosmic accretion and the mechanisms that drive internal gas processing must be routine on $\lesssim 1 \mathrm{Gyr}$ timescales.

We have distinguished three refueling regimes, described as follows. 
1. Blue quasi-bulgeless galaxies are accretion-dominated, enjoying largely continuous refueling and stellar mass growth of order $100 \%$ per Gyr. Contrary to their reputation for "inefficient" star formation, such gas-dominated dwarf galaxies appear to be growing exponentially. We suggest that their high $M_{\mathrm{gas}} / M_{*}$ ratios may be more fairly interpreted as evidence that they are "overwhelmed" rather than "inefficient."

2. Bulged disk galaxies are processing-dominated, consuming a reduced rate of fresh accretion in efficient, often externally driven bursts with net growth rates at tens of percentage levels. Mergers and interactions in this regime create fluctuations in morphology and gas content, including transient blue E/S0 states that may regenerate spiral morphology.

3. Red-and-dead spheroid-dominated galaxies are quenched (per the usual terminology), with negligible gas and $\sim 1 \%-10 \%$ stellar mass growth per Gyr. They may grow slowly by satellite accretion. A population of "nearly quenched" bulged disk galaxies lies at the interface of the processing-dominated and quenched regimes, extending the $M_{\mathrm{gas}} / M_{*}-\mathrm{FSMGR}$ LT relation to low levels.

High SSFRs in dwarf galaxies are sometimes discounted as evidence of bursty star formation, which should average out to lower levels over time. We cannot rule out the possibility of an extremely high mass-to-light ratio, low-SSFR population missed in the V3000 sample due to incompleteness in the SDSS redshift survey. However, we note that $\mathrm{FSMGR}_{\mathrm{LT}}$ is averaged over the past Gyr. Moreover, we have reviewed evidence from recent studies suggesting that dwarf galaxies are not especially bursty, except for the major bursts obviously inherent in dwarfdwarf mergers, such as might produce BCDs. In fact, we have argued that bulged disk galaxies are far more subject to bursty star formation than typical quasi-bulgeless dwarfs. As discussed, the burstiness of such processing-dominated galaxies likely reflects increasingly efficient mechanisms of gas processing (both gas transport and $\mathrm{H}$ I-to- $\mathrm{H}_{2}$ conversion) that develop in tandem with, and partly because of, reduced cosmic accretion.

Connecting back to the threshold and bimodality scales, we have seen that the mass distributions of accretion-dominated, processing-dominated, and quenched galaxies cross at these two scales, suggesting an indirect relationship between refueling and galaxy mass. Focusing on galaxies that are central in their halos, we find a plausible correspondence between these galaxy mass scales and two halo mass scales previously linked to transitions in cosmic accretion $\left(\sim 10^{11.5}\right.$ and $\left.\sim 10^{12.1} M_{\odot}\right)$. In future work considering environment data we will examine the behavior of centrals and satellites separately, demonstrating that centrals evolve through the processing-dominated regime in precisely the narrow mass range between the threshold and bimodality scales. In fact, the V3000 sample demonstrates that "normal" galaxies like our Milky Way_bulged disks with intermediate gas richness - are not in general typical, but most nearly approach typical in this mass range.

Although the analysis in this paper has not used halo mass data, our results clearly motivate the need for a full analysis of $M_{\mathrm{gas}} / M_{*}$ versus $V$ as a function of halo mass and central versus satellite status, for a sample that is highly complete down to galaxy masses below the threshold scale. To date no such sample exists, in that all surveys that probe the full diversity of galaxy environments lack sufficiently sensitive $21 \mathrm{~cm}$ data to probe the full range of $M_{\mathrm{gas}} / M_{*}$ in the dwarf regime. The NFGS offers a glimpse of the power of truly unbiased $\mathrm{H}$ I observations for a broad sample but lacks well-characterized and fairly sampled environments, leading us back to the need for a volume-limited sample with high-quality $21 \mathrm{~cm}$ and kinematic data for a cosmologically diverse range of environments. We aim to construct such a data set as part of the RESOLVE Survey (http://resolve.astro.unc.edu), currently in progress on the SOAR, SALT, Gemini, GBT, and Arecibo telescopes.

We are grateful to Douglas Mar, Jacqueline van Gorkom, John Hibbard, Adam Leroy, Ari Maller, Martha Haynes, Sadegh Khochfar, and Eric Gawiser for illuminating conversations. The anonymous referee provided helpful feedback that improved this manuscript. We thank Perry Berlind, Barbara Carter, Marijn Franx, and the Mount Hopkins observatory staff for their help with the NFGS kinematics observing program. We thank the GBT operators and Green Bank staff for their support of program 10A-070. The National Radio Astronomy Observatory is a facility of the National Science Foundation operated under cooperative agreement by Associated Universities, Inc. S.J.K., D.V.S., K.D.E., and M.A.N. were supported in this research by NSF CAREER grant AST-0955368. D.V.S. and K.D.E. also acknowledge support from GAANN Fellowships and North Carolina Space Grant Fellowships. S.J.K. acknowledges the hospitality and financial assistance of the NRAO Charlottesville visitor program during spring 2010. A.J.M. acknowledges support from a NASA Harriet Jenkins Fellowship, the University of North Carolina Royster Society of Fellows, and the North Carolina Space Grant Program. LHW was supported in part by the NSF under the CARMA cooperative agreement and in part by an SMA Postdoctoral Fellowship. S.J. acknowledges support from the Norman Hackerman Advanced Research Program (NHARP) ARP-03658-0234-2009, NSF grant AST-0607748, and Hubble Space Telescope grant GO-11082 from STScI, which is operated by AURA, Inc., for NASA, under NAS5-26555. This research has made use of the HyperLEDA database (http://leda.univ-lyon1.fr). This work has used the NASA/IPAC Extragalactic Database (NED), which is operated by the Jet Propulsion Laboratory, California Institute of Technology, under contract with the National Aeronautics and Space Administration. This work has made use of data products from the Two Micron All Sky Survey (2MASS), which is a joint project of the University of Massachusetts and the Infrared Processing and Analysis Center/California Institute of Technology, funded by the National Aeronautics and Space Administration and the National Science Foundation. This work is based in part on observations and on archival data obtained with the Spitzer Space Telescope, which is operated by the Jet Propulsion Laboratory, California Institute of Technology under a contract with NASA. Support for this work was provided by an award issued by JPL/Caltech and by NASA. We acknowledge use of the Sloan Digital Sky Survey (SDSS). Funding for SDSS-III has been provided by the Alfred P. Sloan Foundation, the Participating Institutions, the National Science Foundation, and the U.S. Department of Energy Office of Science. The SDSS-III Web site is http://www.sdss3.org/. SDSS-III is managed by the Astrophysical Research Consortium for the Participating Institutions of the SDSS-III Collaboration including the University of Arizona, the Brazilian Participation Group, Brookhaven National Laboratory, University of Cambridge, Carnegie Mellon University, University of Florida, the French Participation Group, the German Participation Group, Harvard University, the Instituto de Astrofisica de Canarias, the Michigan State/Notre Dame/JINA Participation Group, Johns Hopkins University, Lawrence Berkeley National 
Laboratory, Max Planck Institute for Astrophysics, New Mexico State University, New York University, Ohio State University, Pennsylvania State University, University of Portsmouth, Princeton University, the Spanish Participation Group, University of Tokyo, University of Utah, Vanderbilt University, University of Virginia, University of Washington, and Yale University.

\section{REFERENCES}

Abadi, M. G., Navarro, J. F., Steinmetz, M., \& Eke, V. R. 2003, ApJ, 591, 499 Abazajian, K., Adelman-McCarthy, J. K., Agüeros, M. A., et al. 2004, AJ, 128,502

Abazajian, K. N., Adelman-McCarthy, J. K., Agüeros, M. A., et al. 2009, ApJS, 182,543

Adelman-McCarthy, J. K., Agueros, M. A., Allam, S. S., et al. 2008, ApJS, 175,297

Aihara, H., Allende Prieto, C., An, D., et al. 2011, ApJS, 193, 29

Baldry, I. K., Glazebrook, K., Brinkmann, J., et al. 2004, ApJ, 600, 681

Barazza, F. D., Jogee, S., \& Marinova, I. 2008, ApJ, 675, 1194

Barton, E. J., Kannappan, S. J., Kurtz, M. J., \& Geller, M. J. 2000, PASP, 112,367

Barway, S., Kembhavi, A., Wadadekar, Y., Ravikumar, C. D., \& Mayya, Y. D. 2007, ApJL, 661, L37

Barway, S., Wadadekar, Y., Kembhavi, A. K., \& Mayya, Y. D. 2009, MNRAS, 394, 1991

Begum, A., Chengalur, J. N., Karachentsev, I. D., \& Sharina, M. E. 2008, MNRAS, 386, 138

Behroozi, P. S., Wechsler, R. H., \& Conroy, C. 2013, ApJ, 770, 57

Bekki, K. 2008, MNRAS, 388, L10

Bell, E. F. 2008, ApJ, 682, 355

Bell, E. F., McIntosh, D. H., Katz, N., \& Weinberg, M. D. 2003, ApJS, 149, 289

Bettoni, D., Galletta, G., \& García-Burillo, S. 2003, A\&A, 405, 5

Bigiel, F., Leroy, A., Walter, F., et al. 2008, AJ, 136, 2846

Birnboim, Y., \& Dekel, A. 2003, MNRAS, 345, 349

Blanton, M. R., Kazin, E., Muna, D., Weaver, B. A., \& Price-Whelan, A. 2011, AJ, 142, 31

Blanton, M. R., \& Roweis, S. 2007, AJ, 133, 734

Blitz, L., \& Rosolowsky, E. 2006, ApJ, 650, 933

Bouché, N., Dekel, A., Genzel, R., et al. 2010, ApJ, 718, 1001

Brooks, A. M., Governato, F., Booth, C. M., et al. 2007, ApJL, 655, L17

Bruzual, G., \& Charlot, S. 2003, MNRAS, 344, 1000

Bundy, K., Ellis, R. S., \& Conselice, C. J. 2005, ApJ, 625, 621

Burstein, D., Bender, R., Faber, S., \& Nolthenius, R. 1997, AJ, 114, 1365

Calzetti, D. 2001, PASP, 113, 1449

Cardelli, J. A., Clayton, G. C., \& Mathis, J. S. 1989, ApJ, 345, 245

Catinella, B., Schiminovich, D., Kauffmann, G., et al. 2010, MNRAS, 403, 683

Churchill, C. W., Kacprzak, G. G., Steidel, C. C., et al. 2012, ApJ, 760, 68

Colless, M., Peterson, B. A., Jackson, C., et al. 2003, arXiv:astro-ph/0306581

Cortese, L., \& Hughes, T. M. 2009, MNRAS, 400, 1225

Cox, A. L., Sparke, L. S., Watson, A. M., \& van Moorsel, G. 2001, AJ, 121, 692

Dalcanton, J. J. 2007, ApJ, 658, 941

Dalcanton, J. J., Yoachim, P., \& Bernstein, R. A. 2004, ApJ, 608, 189

Davé, R., Finlator, K., \& Oppenheimer, B. D. 2012, MNRAS, 421, 98

Davé, R., Katz, N., Oppenheimer, B. D., Kollmeier, J. A., \& Weinberg, D. H. 2013, MNRAS, 434, 2645

Davies, R. L., Efstathiou, G., Fall, S. M., Illingworth, G., \& Schechter, P. L. 1983, ApJ, 266, 41

Dekel, A., \& Birnboim, Y. 2006, MNRAS, 368, 2

Dekel, A., \& Silk, J. 1986, ApJ, 303, 39

D’Onghia, E., \& Burkert, A. 2004, ApJL, 612, L13

Driver, S. P., Allen, P. D., Liske, J., \& Graham, A. W. 2007, ApJL, 657, L85

Driver, S. P., Hill, D. T., Kelvin, L. S., et al. 2011, MNRAS, 413, 971

Ellison, S. L., Nair, P., Patton, D. R., et al. 2011, MNRAS, 416, 2182

Emsellem, E., Cappellari, M., Krajnović, D., et al. 2007, MNRAS, 379, 401

Falco, E. E., Kurtz, M. J., Geller, M. J., et al. 1999, PASP, 111, 438

Ferreras, I., Silk, J., Böhm, A., \& Ziegler, B. 2004, MNRAS, 355, 64

Feulner, G., Goranova, Y., Drory, N., Hopp, U., \& Bender, R. 2005, MNRAS, 358, L1

Fisher, D. B., \& Drory, N. 2011, ApJL, 733, L47

Fontanot, F., De Lucia, G., Wilman, D., \& Monaco, P. 2011, MNRAS, 416, 409

Franx, M., van Dokkum, P. G., Schreiber, N. M. F., et al. 2008, ApJ, 688, 770

Fraternali, F., \& Tomassetti, M. 2012, MNRAS, 426, 2166

Gardan, E., Braine, J., Schuster, K. F., Brouillet, N., \& Sievers, A. 2007, A\&A, 473,91
Garnett, D. R. 2002, ApJ, 581, 1019

Geha, M., Blanton, M., Yan, R., \& Tinker, J. 2012, ApJ, 757, 85

Giavalisco, M., Vanzella, E., Salimbeni, S., et al. 2011, ApJ, 743, 95

Giovanelli, R., Haynes, M. P., Kent, B. R., et al. 2005, AJ, 130, 2598

Giovanelli, R., Haynes, M. P., Salzer, J. J., et al. 1994, AJ, 107, 2036

Gonzalez-Perez, V., Castander, F. J., \& Kauffmann, G. 2011, MNRAS, 411,1151

Governato, F., Willman, B., Mayer, L., et al. 2007, MNRAS, 374, 1479

Hawarden, T. G., Longmore, A. J., Goss, W. M., Mebold, U., \& Tritton, S. B. 1981, MNRAS, 196, 175

Haynes, M. P., \& Giovanelli, R. 1984, AJ, 89, 758

Haynes, M. P., Giovanelli, R., Martin, A. M., et al. 2011, AJ, 142, 170

Hopkins, P. F., Kereš, D., Ma, C.-P., \& Quataert, E. 2010, MNRAS, 401, 1131

Hopkins, P. F., Somerville, R. S., Cox, T. J., et al. 2009, MNRAS, 397, 802

Huang, S., Haynes, M. P., Giovanelli, R., \& Brinchmann, J. 2012, ApJ, 756, 113

Huchra, J., Davis, M., Latham, D., \& Tonry, J. 1983, ApJS, 52, 89

Huertas-Company, M., Aguerri, J. A. L., Tresse, L., et al. 2010, A\&A, 515, A3 Isobe, T., Feigelson, E. D., \& Nelson, P. I. 1986, ApJ, 306, 490

Jansen, R. A., Fabricant, D., Franx, M., \& Caldwell, N. 2000a, ApJS, 126, 331 Jansen, R. A., Franx, M., Fabricant, D., \& Caldwell, N. 2000b, ApJS, 126, 271

Jarrett, T. H., Chester, T., Cutri, R., et al. 2000, AJ, 119, 2498

Jones, D. H., Read, M. A., Saunders, W., et al. 2009, MNRAS, 399, 683

Kannappan, S. J. 2004, ApJL, 611, L89 (K04)

Kannappan, S. J., \& Barton, E. J. 2004, AJ, 127, 2694

Kannappan, S. J., \& Fabricant, D. G. 2001, AJ, 121, 140

Kannappan, S. J., Fabricant, D. G., \& Franx, M. 2002, AJ, 123, 2358 (KFF)

Kannappan, S. J., \& Gawiser, E. 2007, ApJL, 657, L5

Kannappan, S. J., Guie, J. M., \& Baker, A. J. 2009, AJ, 138, 579 (KGB)

Kannappan, S. J., Jansen, R. A., \& Barton, E. J. 2004, AJ, 127, 1371

Kannappan, S. J., \& Wei, L. H. 2008, in AIP Conf. Proc. 1035, The Evolution of Galaxies through the Neutral Hydrogen Window, ed. R. Minchin \& E. Momjian (Melville, NY: AIP), 163

Kassin, S. A., Weiner, B. J., Faber, S. M., et al. 2007, ApJL, 660, L35

Kauffmann, G., Heckman, T. M., De Lucia, G., et al. 2006, MNRAS, 367, 1394

Kauffmann, G., Heckman, T. M., White, S. D. M., et al. 2003a, MNRAS, 341,54

Kauffmann, G., Heckman, T. M., White, S. D. M., et al. 2003b, MNRAS, 341,33

Kereš, D., Katz, N., Weinberg, D. H., \& Davé, R. 2005, MNRAS, 363, 2

Khochfar, S., \& Silk, J. 2011, MNRAS, 410, L42

Krumholz, M. R., \& Dekel, A. 2012, ApJ, 753, 16

Krumholz, M. R., Leroy, A. K., \& McKee, C. F. 2011, ApJ, 731, 25

Kurtz, M. J., Mink, D. J., Wyatt, W. F., et al. 1992, in ASP Conf. Ser. 25, Astronomical Data Analysis Software and Systems I, ed. D. M. Worrall, C. Biemesderfer, \& J. Barnes (San Francisco, CA: ASP), 432

La Barbera, F., De Carvalho, R. R., De La Rosa, I. G., et al. 2010, AJ, 140,1528

Laurikainen, E., Salo, H., Buta, R., Knapen, J. H., \& Comerón, S. 2010, MNRAS, 405, 1089

Lavalley, M. P., Isobe, T., \& Feigelson, E. D. 1992, BAAS, 24, 839

Leaman, R., Cole, A. A., Venn, K. A., et al. 2009, ApJ, 699, 1

Leaman, R., Venn, K. A., Brooks, A. M., et al. 2012, ApJ, 750, 33

Leauthaud, A., Tinker, J., Bundy, K., et al. 2012, ApJ, 744, 159

Lee, J. C., Gil de Paz, A., Kennicutt, R. C., Jr., et al. 2011, ApJS, 192, 6

Lee, J. C., Kennicutt, R. C., Jr., Funes, S. J. J. G., Sakai, S., \& Akiyama, S. 2009, ApJ, 692, 1305

Leitner, S. N., \& Kravtsov, A. V. 2011, ApJ, 734, 48

Li, C., Kauffmann, G., Fu, J., et al. 2012, MNRAS, 424, 1471

Lu, Y., Kereš, D., Katz, N., et al. 2011, MNRAS, 416, 660

Mac Low, M., \& Ferrara, A. 1999, ApJ, 513, 142

Madden, S. C., Poglitsch, A., Geis, N., Stacey, G. J., \& Townes, C. H. 1997, ApJ, 483, 200

Maller, A. H., Katz, N., Kereš, D., Davé, R., \& Weinberg, D. H. 2006, ApJ, 647, 763

Marino, A., Bianchi, L., Rampazzo, R., et al. 2011, ApJ, 736, 154

Martig, M., Bournaud, F., Teyssier, R., \& Dekel, A. 2009, ApJ, 707, 250

Martin, A. M., Papastergis, E., Giovanelli, R., et al. 2010, ApJ, 723, 1359

Martin, C. L., Shapley, A. E., Coil, A. L., et al. 2012, ApJ, 760, 127

Martini, P., Dicken, D., \& Storchi-Bergmann, T. 2013, ApJ, 766, 121

Mazzarella, J. M., \& Boroson, T. A. 1993, ApJS, 85, 27

McGaugh, S. S. 2005, ApJ, 632, 859

McGaugh, S. S., Schombert, J. M., Bothun, G. D., \& de Blok, W. J. G. 2000, ApJL, 533, L99

Misgeld, I., \& Hilker, M. 2011, MNRAS, 414, 3699

Moffett, A. J., Kannappan, S. J., Baker, A. J., \& Laine, S. 2012, ApJ, 745,34 
Morganti, R., de Zeeuw, P. T., Oosterloo, T. A., et al. 2006, MNRAS, 371,157

Morrissey, P., Conrow, T., Barlow, T. A., et al. 2007, ApJS, 173, 682

Moster, B. P., Naab, T., \& White, S. D. M. 2013, MNRAS, 428, 3121

Mulchaey, J. S., \& Jeltema, T. E. 2010, ApJL, 715, L1

Nair, P. B., \& Abraham, R. G. 2010, ApJL, 714, L260

Narayanan, A., Wakker, B. P., Savage, B. D., et al. 2010, ApJ, 721, 960

Navarro, J. F., \& White, S. D. M. 1994, MNRAS, 267, 401

Nelson, D., Vogelsberger, M., Genel, S., et al. 2013, MNRAS, 429, 3353

O’Donnell, J. E. 1994, ApJ, 422, 158

Papastergis, E., Martin, A. M., Giovanelli, R., \& Haynes, M. P. 2011, ApJ, 739, 38

Paturel, G., Petit, C., Prugniel, P., et al. 2003, A\&A, 412, 45

Pelupessy, F. I., \& Papadopoulos, P. P. 2009, ApJ, 707, 954

Peng, Y.-j., Lilly, S. J., Renzini, A., \& Carollo, M. 2012, ApJ, 757, 4

Pfenniger, D., \& Revaz, Y. 2005, A\&A, 431, 511

Pisano, D. J., Kobulnicky, H. A., Guzmán, R., Gallego, J., \& Bershady, M. A. 2001, AJ, 122, 1194

Pustilnik, S. A., Kniazev, A. Y., Lipovetsky, V. A., \& Ugryumov, A. V. 2001, A\&A, 373, 24

Raychaudhury, S., von Braun, K., Bernstein, G. M., \& Guhathakurta, P. 1997, AJ, 113, 2046

Reyes, R., Mandelbaum, R., Gunn, J. E., et al. 2012, MNRAS, 425, 2610

Robertson, B., Bullock, J. S., Cox, T. J., et al. 2006, ApJ, 645, 986

Sage, L. J., \& Welch, G. A. 2006, ApJ, 644, 850

Salim, S., Dickinson, M., Michael Rich, R., et al. 2009, ApJ, 700, 161

Salim, S., Fang, J. J., Rich, R. M., Faber, S. M., \& Thilker, D. A. 2012, ApJ, 755,105

Salim, S., \& Rich, R. M. 2010, ApJL, 714, L290

Salim, S., Rich, R. M., Charlot, S., et al. 2007, ApJS, 173, 267 (S07)

Sancisi, R., Fraternali, F., Oosterloo, T., \& van der Hulst, T. 2008, A\&ARv, 15,189

Schawinski, K., Lintott, C., Thomas, D., et al. 2009, MNRAS, 396, 818

Schawinski, K., Urry, C. M., Virani, S., et al. 2010, ApJ, 711, 284

Schlegel, D. J., Finkbeiner, D. P., \& Davis, M. 1998, ApJ, 500, 525
Schruba, A., Leroy, A. K., Walter, F., et al. 2011, AJ, 142, 37

Serra, P., Trager, S. C., Oosterloo, T. A., \& Morganti, R. 2008, A\&A, 483, 57

Sheth, K., Regan, M., Hinz, J. L., et al. 2010, PASP, 122, 1397

Sinha, M., \& Holley-Bockelmann, K. 2012, ApJ, 751, 17

Somerville, R. S., Hopkins, P. F., Cox, T. J., Robertson, B. E., \& Hernquist, L. 2008, MNRAS, 391, 481

Spavone, M., Iodice, E., Arnaboldi, M., et al. 2010, ApJ, 714, 1081

Stanonik, K., Platen, E., Aragón-Calvo, M. A., et al. 2009, ApJL, 696, L6

Stark, D. V., Kannappan, S. J., Wei, L. H., et al. 2013, ApJ, 769, 82 (S13)

Stewart, K. R. 2011, arXiv:1109.3207

Stewart, K. R., Bullock, J. S., Wechsler, R. H., \& Maller, A. H. 2009, ApJ, 702,307

Stewart, K. R., Bullock, J. S., Wechsler, R. H., Maller, A. H., \& Zentner, A. R. 2008, ApJ, 683, 597

Stewart, K. R., Kaufmann, T., Bullock, J. S., et al. 2011, ApJ, 738, 39

Thilker, D. A., Bianchi, L., Schiminovich, D., et al. 2010, ApJL, 714, L171

Tremonti, C. A., Heckman, T. M., Kauffmann, G., et al. 2004, ApJ, 613,898

van den Bergh, S. 1976, ApJ, 206, 883

van der Marel, R. P., \& Franx, M. 1993, ApJ, 407, 525

van de Voort, F., Schaye, J., Booth, C. M., Haas, M. R., \& Dalla Vecchia, C. 2011, MNRAS, 414, 2458

Wei, L. H., Kannappan, S. J., Vogel, S. N., \& Baker, A. J. 2010a, ApJ, 708, 841 (W10a)

Wei, L. H., Vogel, S. N., Kannappan, S. J., et al. 2010b, ApJL, 725, L62

Weil, M. L., Eke, V. R., \& Efstathiou, G. 1998, MNRAS, 300, 773

Weinzirl, T., Jogee, S., Khochfar, S., Burkert, A., \& Kormendy, J. 2009, ApJ, 696,411

Weisz, D. R., Johnson, B. D., Johnson, L. C., et al. 2012, ApJ, 744, 44

Woo, J., Dekel, A., Faber, S. M., et al. 2013, MNRAS, 428, 3306

Yoachim, P., \& Dalcanton, J. J. 2005, ApJ, 624, 701

Zhang, W., Li, C., Kauffmann, G., et al. 2009, MNRAS, 397, 1243

Zitrin, A., \& Brosch, N. 2008, MNRAS, 390, 408 